\title{
SPECTRAL LINE FORMATION IN HOT JUPITER ATMOSPHERES AND IN
} INTERACTING SUPERNOVAE

\author{
Chenliang Huang (黄辰亮) \\ Beijing, China
}

B.S., Peking University, 2011

M.S., University of Virginia, 2013

\begin{abstract}
A Dissertation Presented to the Graduate
Faculty of the University of Virginia in Candidacy for the Degree of Doctor of Philosophy
\end{abstract}
Department of Astronomy
University of Virginia
August, 2017

Philip L. Arras

Roger A. Chevalier

Shane W. Davis

Robert E. Johnson 
(c) Copyright by

Chenliang Huang (黄辰亮)

All rights reserved

August, 2017 


\begin{abstract}
A myriad of information about the physical properties of an astronomical object, such as its composition, temperature, ionization state, velocity, redshift and more, are embedded in its spectral lines. To obtain this information from the observed spectrum, one needs to understand the formation of these spectral lines in the system. In this thesis, I present the spectral line formation study of two systems by comparing the simulated spectral line from theoretical models to the observations. In the first part of the thesis (Chapters 1 and 2), I investigate $\mathrm{H} \alpha$ and $\mathrm{NaD}$ lines in the hot Jupiter transmission spectrum, which are formed due to absorption in the planetary upper atmosphere. In the second part (Chapter 3), I study the broadening of narrow emission lines of interacting supernovae due to electron scattering.

When a planet transits in front of its host star, the atmosphere can absorb an extra part of the light from the star besides the planet itself. This frequency dependent absorption of starlight by the planet atmosphere, referred as a transmission spectrum, provides a probe of the atmospheric composition and structure. The $\mathrm{H} \alpha$ and $\mathrm{NaD}$ transmission spectra have been observed for the hot Jupiter HD 189733b, which may play an important role in understanding the conditions in the planet's atomic layer. Motivated by the observations, a detailed one-dimensional hydrostatic atmosphere model is constructed over the region dominated by atomic hydrogen, and comparison of model transmission spectra to the data has been made. An atomic hydrogen level population calculation and a Monte-Carlo Ly $\alpha$ radiation transfer simulation are carried out to model the abundance of $2 \ell$ state hydrogen. The dependence of the transit profile on Lyman continuum ( $\mathrm{LyC}$ ) emission and metal abundances is considered. The atmospheric temperature of the model is compared to previous temperature measurements from the Na line profile.
\end{abstract}


Interacting supernovae, including type IIn and Ia-CSM, are supernovae that show evidence of strong shock interaction between their ejecta and pre-existing circumstellar material (CSM), which may be ejected from the unstable progenitor star before the explosion. After the supernova shock wave has broken out of the progenitor star, the ionizing radiation from the shock region is able to ionize the surroundings. The CSM can have substantial optical depth to electron scattering and the continuum photosphere is in the unshocked CSM due to the electron scattering opacity. The iconic feature of the interacting supernovae are the broad wings (1000's of $\mathrm{km} \mathrm{s}^{-1}$ ) on narrow emission lines. To explain the line formation of this feature, we adopt the idea that the narrow emissions are created in the preshock ionized CSM by recombination cascades, and the line profiles are broadened by the electron scattering for the photons that make their way out. A Monte-Carlo simulation has been done to simulate this electron scattering process in a series of possible CSM configurations. The dependence of the line profile on the optical depth, thickness, density distribution, expansion velocity, and continuum absorption has been examined. The scattering model has been applied to a number of supernovae, including Type IIn and Type Ia-CSM events. 


\section{Acknowledgements}

First and foremost, I would like to express my sincere gratitude to my advisor, Phil Arras, without whom this dissertation would have never been possible. I have greatly benefited from his broad knowledge and vision of the field. He has not only devoted enormous effort and time to every aspect of our research, but he has also offered me plenty of advice for my career. His striving for excellence and rigorous approach has set an outstanding example of a scientist and person for me. It is my great honor to be his Ph.D. student. I want to express the same gratefulness to Roger Chevalier, who led me into the academic study with extraordinary insight and patience. He supervised our project on supernova, which was my first research project in the true sense. I also thank the other members of my thesis committee, Shane Davis and Bob Johnson, for their inspiring discussion and invaluable advice. I gratefully acknowledge Duncan Christie, who explained his work on the exoplanetary atmosphere model to me in great detail, which is the ground stone of this thesis. It was a pleasure to work with Jake Borish on the SN 2010jl project, though the work is not included in this thesis. I acknowledge Katherine Holcomb, whose constructive advice considerably improved the efficiency of my code. I give special thanks to ZhiYun Li, whose important advice has guided me in the right direction. Thank you for inviting us to your house with delicious food. And it is my pleasure to have been your soccer friend for six years.

In addition, I want to say thanks to C. David Rigby for all the quick technical supports at any time. To my office-mates, Dom Pesce, Scott Suriano, Nick Troup, Haifeng Yang, and Apurva Oza, I enjoy the environment you created, and the fruitful discussion with you. You are always willing and ready to help me, from solving my language question to accompanying me with a policeman to meet the hawker who 
was selling my stolen bike. To Bin Chen, Bo Zhao, and Xiaping Tang, for helping me to adapt to the new environment faster. To other classmates in my year, Brian Prager, Cheoljong Lee, and John Allan, for the help and company in finishing the coursework in the first two years. To all the other faculty, staff, and classmates in the department with whom I spent six years of joyful life at UVa. To Xiaopu Li, my four-year roommate, for being an amazing friend and for all the fun moments we had.

Finally, I express my deepest gratitude and love to my family. My parents and grandparents inspired my interest in the natural science, encouraged me to explore unknowns, and consistently supported my interests. It was you who directed me to this academic path. Since my childhood, your enthusiasm for research has had a profound influence on me. I am very proud and fortunate to have a dad who is willing to discuss my research project. I also have to thank my aunt, who help me to prepare for my life abroad. At last, to my wife, Meng Sun. You have made my life in graduate school so special and especially memorable. Thank you for sharing all the highs and lows, and for the cheering and encouragement. It would not have been nearly as easy or as much fun without you. 


\section{Table of Contents}

Abstract $\quad$ i

Acknowledgements $\quad$ iii

List of Figures $\quad$ viii

List of Tables $\quad$ ix

1 Introduction to the Atmosphere of HD 189733b 1

1.1 Study of the Atmosphere of an Exoplanet Using the Transmission Spectrum of Atomic Resonance Lines . . . . . . . . . . . . . . 1

1.2 Observations of the Upper Atmosphere of HD 189733b $\ldots \ldots \ldots$

1.3 Project Overview . . . . . . . . . . . . . . . . . . . . 16

$2 \quad \mathrm{H} \alpha$ and NaD Transmission Spectrum of HD 189733b 19

2.1 Analytic Estimates . . . . . . . . . . . . . . . . . . . . . . . 19

2.2 Hydrogen Level Population . . . . . . . . . . . . . . . . . . . . . . 24

2.3 The Atmosphere Model . . . . . . . . . . . . . . . . . . . . . . . . 30

2.3 .1 Basic Structure . . . . . . . . . . . . . . . . . . 30

2.3.2 Differential Equations . . . . . . . . . . . . . . . . 31

2.3.3 Ionization State and Temperature . . . . . . . . . . . . . 32

2.3.4 Radiative Cooling Due to Metal Species . . . . . . . . . . . 35

2.3.5 Molecular Hydrogen . . . . . . . . . . . . . . . . . 38

2.4 Lyo Radiation Transfer . . . . . . . . . . . . . . . . . . . . . . . . . 40

2.5 Fiducial Atmosphere Model . . . . . . . . . . . . . . . . . . . . 44

2.6 Transmission Spectrum . . . . . . . . . . . . . . . . . . 56

2.6.1 $\mathrm{H} \alpha$ Transmission Spectrum _. . . . . . . . . . . . 56

2.6.2 NaD Transmission Spectrum . . . . . . . . . . . . . . . 61

2.6.3 Retrieval of the Temperature Profile from NaD Transmission Spectra . . . . . . . . . . . . . . . 6 6 65

2.6.4 Impact of LyC Flux on Transit Depth . . . . . . . . . . . 71

2.6.5 Impact of Metallicity on Transit Depth . . . . . . . . . . . 72

2.7 Discussion . . . . . . . . . . . . . . . . . . . . . . . . . . . 72 
2.7.1 Other Possible Cooling Mechanisms . . . . . . . . . . . . . . . 72

2.7.2 Comparing with Other Hot Jupiter Upper Atmosphere Models 74

2.8 Conclusion . . . . . . . . . . . . . . . . 76

3 Electron Scattering Wings on Lines in Interacting Supernovae 81

3.1 Introduction . . . . . . . . . . . . . . . . . 81

3.2 Scattering in a Circumstellar Medium . . . . . . . . . . . . 83

3.2.1 The Single Scattering Limit . . . . . . . . . . . . . . . . . 85

3.2.2 Stationary Circumstellar Medium . . . . . . . . . . . . . 85

3.2.3 Presupernova Mass Loss Velocity . . . . . . . . . . . . . . . . 92

3.2.4 Radiative Acceleration of Circumstellar Gas . . . . . . . . . . 97

3.2.5 Effects of Continuum Absorption . . . . . . . . . . . . . . 99

3.3 Comparison with Observations . . . . . . . . . . . . . . 100

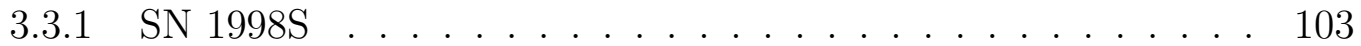

3.3.2 SN 2005cl, SN 2005db, and SN 2012bq . . . . . . . . . . 107

3.3.3 SN 2005gj, SN 2008J, and SN 2008cg . . . . . . . . . . . 109

3.3.4 SN 2009ip . . . . . . . . . . . . . . . . . . . 114

3.3.5 SN 2010jl . . . . . . . . . . . . . . . . . . . . . . 114

3.3.6 SN 2011ht . . . . . . . . . . . . . . . . 116

3.4 Discussion and Conclusions _. . . . . . . . . . . . . . . 118

4 Summary and Future Work 122

4.1 Summary . . . . . . . . . . . . . . . . . . . . . . 122

4.2 Future Work . . . . . . . . . . . . . . . . . 125

4.2.1 Temperature Profile Retrieval . . . . . . . . . . . . . . . . . 125

4.2.2 Solving the Ly $\alpha$ Resonant Scattering Analytically. . . . . . . . 126

4.2.3 Systematic Parameter Search and Simulating Other Observable Atomic Lines. . . . . . . . . . . . . . . . . . . . . . . 127

4.2.4 Simulate the Ly $\alpha$ and FUV Flux Reaching the Molecular Layer 128

4.2.5 Extending the Current Atmosphere Model to 3D. . . . . . . . 129

4.2.6 Explaining the $\mathrm{H} \alpha$ Absorption Leading the Transit. . . . . . . 129

$\begin{array}{ll}\text { References } & 131\end{array}$ 


\section{List of Figures}

$1.1 M \sin (i)$ vs semi-major axis for confirmed exoplanets . . . . . . . . 2

1.2 Illustration of the transmission spectrum . . . . . . . . . . . . . . 4

1.3 Illustration of transmission spectrum view from the side . . . . . . . 5

$1.4 \mathrm{NaD}$ transmission spectrum . . . . . . . . . . . . . . . . . . . . . . . . . . . . 12

$1.5 \mathrm{H} \alpha$ transmission spectrum . . . . . . . . . . . . . . . 13

2.1 Adopted stellar spectrum of HD 189733b . . . . . . . . . . . . 25

2.2 Ly $\alpha$ mean intensity spectrum in the atmosphere . . . . . . . . . . . . . . 45

2.3 Temperature and optical depth profile . . . . . . . . . . . . . 46

2.4 Cooling rate . . . . . . . . . . . . . . . . . 47

2.5 Number density of main species . . . . . . . . . . . . . . . . . . . . . . . . . . 48

2.6 Ly $\alpha$ photon sources and sinks . . . . . . . . . . . . . . . . . . . 55

2.7 Line profile weighted $\operatorname{Ly} \alpha$ mean intensity . . . . . . . . . . . . . . 56

2.8 Reliability of the hydrogen excitation approximation . . . . . . . 57

$2.9 \mathrm{H} \alpha$ transmission spectrum . . . . . . . . . . . . . . . . . 58

2.10 Line center optical depth vs impact parameter . . . . . . . . . . 59

2.11 Equivalent width per unit impact parameter vs impact parameter . . 60

2.12 Comparison of $\mathrm{NaD}$ transmission spectrum with the observation by Huitson et al. (2012) . . . . . . . . . . . . . . . 62

2.13 Comparison of $\mathrm{NaD}$ transmission spectrum with the observation by Wyttenbach et al. (2015) . . . . . . . . . . . . 63

2.14 Fitting with the isothermal atmosphere model . . . . . . . . . . 67

2.15 Retrieved temperature profile using isothermal atmosphere model . . 68

3.1 Illustration of the scattering region . . . . . . . . . . . . 84

3.2 Line profile for single scattering . . . . . . . . . . . . . 86

3.3 Line profiles of static circumstellar mediums . . . . . . . . . . . . 88

3.4 Normalized line shapes of static circumstellar mediums . . . . . . . 89

3.5 Relation of FWHM vs. $\tau$. . . . . . . . . . . . . . . . . 90

3.6 Line profiles for a series of outer to inner radius ratios . . . . . . . . . 91

3.7 Relation of narrow to total line ratio vs. $\tau$. . . . . . . . . . . . 92

3.8 Narrow to total line ratio vs. the power law of the density profile . . 93

3.9 FWHM vs. the power law of the density profile . . . . . . . . . . 94 
3.10 Line profiles for mediums with constant outflow velocity . . . . . . 95

3.11 Line profiles for radiative accelerated mediums . . . . . . . . . . . 98

3.12 The variation of the line profile with continuum absorption . . . . . . 100

3.13 Comparison of the model with the SN 1998S H $\alpha$ emission line on 1998 March 4 . . . . . . . . . . . . . . . . . 105

3.14 Comparison of the model with the SN 1998S H $\alpha$ emission line on 1998 March 6 . . . . . . . . . . . . . . . . . . . . 106

3.15 Comparison of the model with the SN 2005cl H $\alpha$ emission line . . . . 108

3.16 Comparison of the model with the SN 2005db H $\alpha$ emission line . . . 109

3.17 Comparison of the model with the SN 2012bq H $\alpha$ emission line . . . 110

3.18 Comparison of the model with the SN 2005gj H $\alpha$ emission line . . . . 111

3.19 Comparison of the model with the SN 2008J H $\alpha$ emission line . . . . 112

3.20 Comparison of the model with the SN 2008cg H $\alpha$ emission line . . . . 113

3.21 Comparison of the model with the SN 2009ip H $\alpha$ emission line . . . . 115

3.22 Comparison of the model with the SN 2010jl Pa $\beta$ emission line . . . . 116

3.23 Comparison of the model with the SN 2011ht $\mathrm{H} \alpha$ emission line . . . . 117 


\section{List of Tables}

1.1 Orbital and physical parameters of HD 189733 and HD 189733b . . . 9

2.1 Photoionization rates and photoelectric heating rates . . . . . . . . 29

2.2 Rate which may be important to $n_{2 p}$ population and de-population . 29

2.3 List of major metal cooling transitions . . . . . . . . . . . . . . 79

2.4 List of minor metal cooling transitions . . . . . . . . . . . . . . 80

3.1 Supernova observational parameters . . . . . . . . . . . . . 120

3.2 Supernova model parameters used in fits . . . . . . . . . . . . . . 121 


\section{Chapter 1}

\section{Introduction to the Atmosphere of}

\section{HD 189733b}

\subsection{Study of the Atmosphere of an Exoplanet Us- ing the Transmission Spectrum of Atomic Res- onance Lines}

There are nearly 3000 confirmed detections of planets orbiting other stars, and more than 2000 additional candidates have been identified ${ }^{1}$. Figure 1.1 shows the minimum planet mass, $M \sin (i)$, against the orbital semi-major axis of planets which have a mass measurement as of Feb. 21, 2016. The discovered exoplanets and exoplanetary systems have a wide diversity in the parameter space, largely unexpected from the observation of our own solar system.

The ultimate goal of the study of exoplanets is to answer the ancient question, "Are we alone?" An understanding of the exoplanet atmosphere is crucial for inves-

\footnotetext{
${ }^{1}$ http://exoplanets.org/
} 


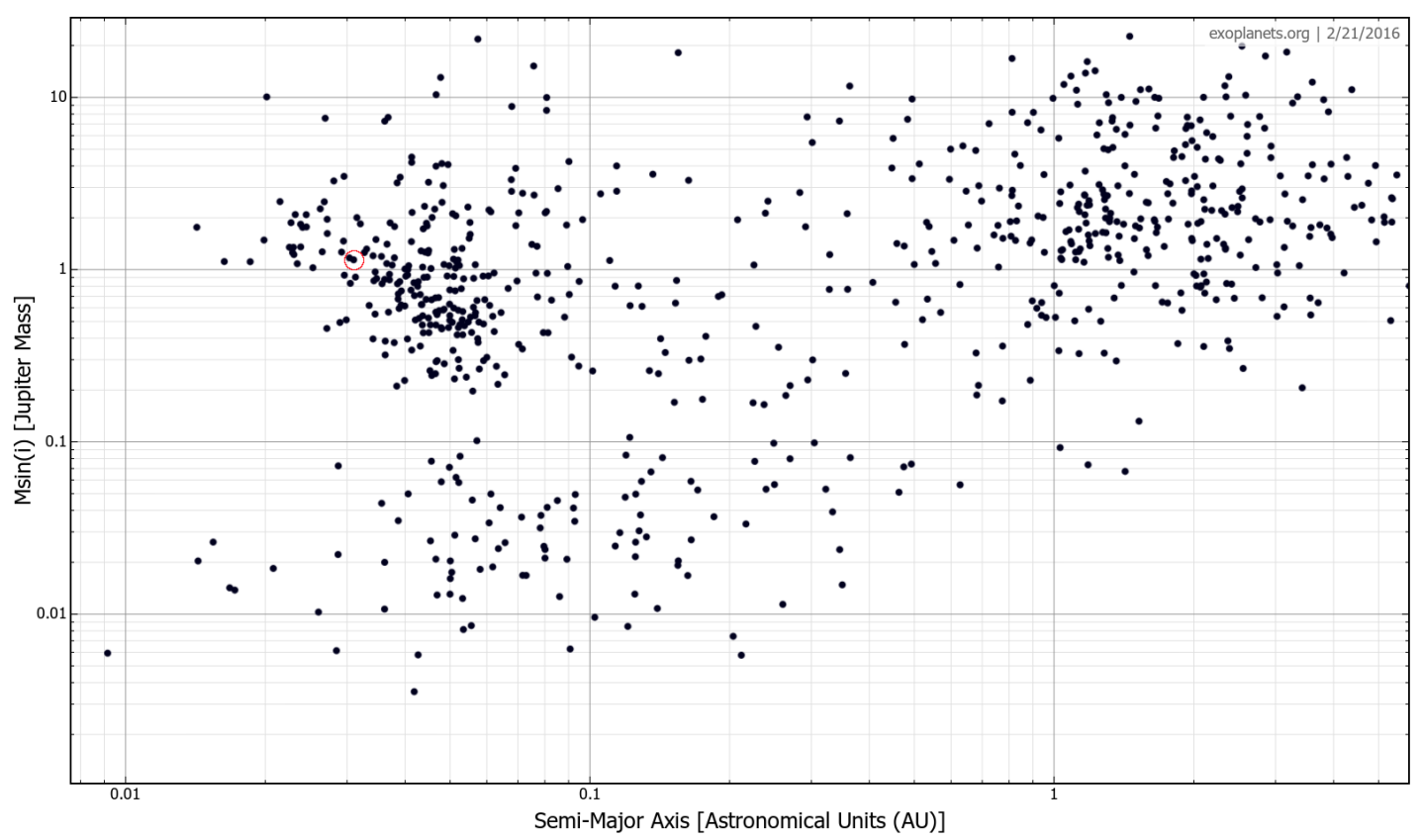

Fig. 1.1. - Minimum mass, $M \sin (i)$, vs semi-major axis for confirmed exoplanets ${ }^{2}$. The hot Jupiter HD 189733b is marked with the small red circle.

tigating the habitability of a planet. Almost all of the information about planetary atmosphere temperature and chemical abundance comes from atmospheric photometry or spectroscopy (Seager 2010). Understanding a planet's atmosphere is also a necessary condition for understanding its formation, structure, and evolution (Burrows 2014b).

The most straightforward way to pursue this question is by taking an image of the exoplanet, so-called direct imaging, as is used to study the planets in the solar system. Because of the close distance and extremely large brightness contrast between the planet and its host star, the light of the planet and star cannot be separately resolved using current technology, except for a few dozen young giant planets at wide orbital distance which have been imaged directly (Bowler 2016).

So far, the most effective method of characterizing exoplanet atmosphere is using

${ }^{2}$ http://exoplanets.org/ 
the transit method, which has been described as the "royal road" of exoplanetary science (Winn 2010). A transit is when a small celestial body passes in front of a larger body and obscures the light of the larger body. An exoplanet transit can be observed if the orbit is viewed edge-on. The majority of the exoplanets discovered so far are found by the Kepler Mission, looking for the dimming of the star's light caused by the exoplanet transit.

A planet with a shorter orbital period is easier to detect with the transit method. It is not only because it transits more frequently, but also because its closer distance to the star leads to a larger transit probability. A larger planet radius compared to the host star can obscure a larger area of the star and creates a larger dimming of the stellar brightness. Thus, a giant planet, which usually means a more massive planet, is also preferred for the transit method.

Hot Jupiters are a category of discovered exotic worlds that very different from any planets in the Solar System. These are giant planets like Jupiter but much hotter, with $M \sin (i) \gtrsim 0.5 M_{\text {Jup }}$ and orbital period $P \lesssim 10$ days, shown in the upper left corner of the Figure 1.1. For a solar mass host star, $P=10$ days corresponds to an semi-major axis $a=0.09$ AU. Therefore, hot Jupiters are the planets that are easiest to be detected and to have a mass measurement using the radial-velocity method. Although hot Jupiters are not as common, relative to smaller or more distant planets, as they look in the plot because of these selection effects, a couple of hundreds discovered hot Jupiters indicate that they are not oddballs.

The higher signal to noise transit detections of nearby hot Jupiters provide the opportunity to measure the transit depth as a function of wavelength, also known as the transmission spectrum, which can be used to probe its atmosphere. The atmosphere of the Earth is the optically thin gas above the solid surface. However, 
the solid core of a gas giant is embedded deeply inside and cannot be observed. Thus, the atmosphere of a gas giant stands for the layer above the planet surface at a radius $R_{p}$, which is defined by the broadband optical transit radius (Burrows 2014b).

Figures 1.2 and 1.3 illustrate the concept of the transmission spectrum. The stellar beams pointed at the Earth probe the transiting planet's atmosphere transversely along a chord perpendicular to the impact radius (Figure 1.3). Besides the opaque planet, the atmosphere blocks a fraction of $1-e^{-\tau_{\nu}}$ of the stellar intensity, where $\tau_{\nu}$ is the optical depth at frequency $\nu$ along the chord associated with the impact parameter b. Because the opacity of molecules and atoms in a planet's atmosphere is a function of wavelength, which contributes extra obscuration to the stellar light, the transmission spectrum can be used to characterize the atmosphere near the planet's terminator. The ability to detect a planet's atmosphere using the transmission spectrum is shown by Charbonneau et al. (2002), who were the first to successfully use this technique with the $4 \sigma$ measurement of atomic sodium in the atmosphere of a nearby hot Jupiter HD 209458b.

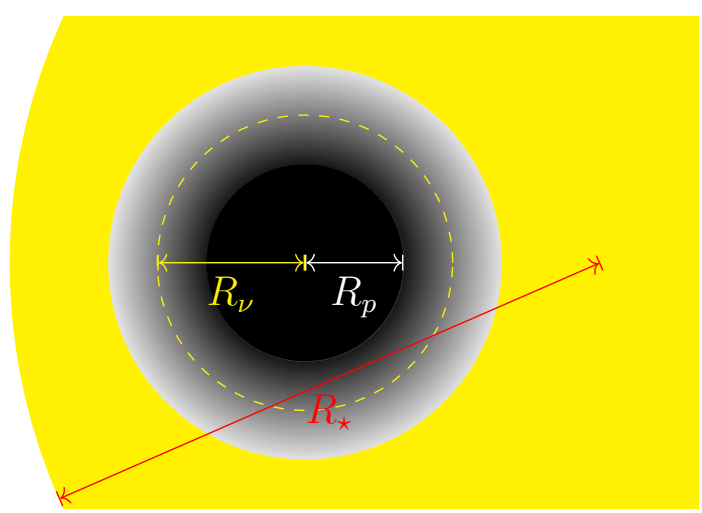

Fig. 1.2. - Illustration of the transmission spectrum. The yellow block in the background indicates the surface of the host star with radius $R_{\star}$. The center black circle with radius $R_{p}$ shows the opaque body of a transiting planet. The surrounding shading annulus shows the atmosphere of the planet, which absorbs the stellar light during transit in a frequency-dependent manner. The yellow dash circle indicates the apparent size of the planet at a certain frequency. 
For a planet at distance $d$ from the observer, with uniform intensity $I_{\nu}$ over the stellar disk, the measured flux is

$$
F_{\nu}=\frac{2 \pi I_{\nu}}{d^{2}} \int_{0}^{R_{\star}} e^{-\tau_{\nu}(b)} b \mathrm{~d} b
$$

The optical depth can be divided into a continuum part, $\tau_{c}(b)$, which is independent of frequency over the line, and the line opacity part due to atomic or molecular absorption lines,

$$
\tau_{l, \nu}(b)=2 \int_{0}^{\sqrt{R_{\mathrm{top}}^{2}-b^{2}}} n \sigma_{\nu} \mathrm{d} s,
$$

where $s$ is the line of sight distance and $\sigma_{\nu}$ is the line absorption cross section. The continuum absorption is then complete for $b<R_{p}$ and zero for $b>R_{p}$. The continuum integral then becomes as

$$
F_{\nu}^{(c)}=\frac{2 \pi I_{\nu}}{d^{2}} \int_{0}^{R_{\star}} e^{-\tau_{c}} b \mathrm{~d} b=I_{\nu} \frac{\pi\left(R_{\star}^{2}-R_{p}^{2}\right)}{d^{2}} .
$$
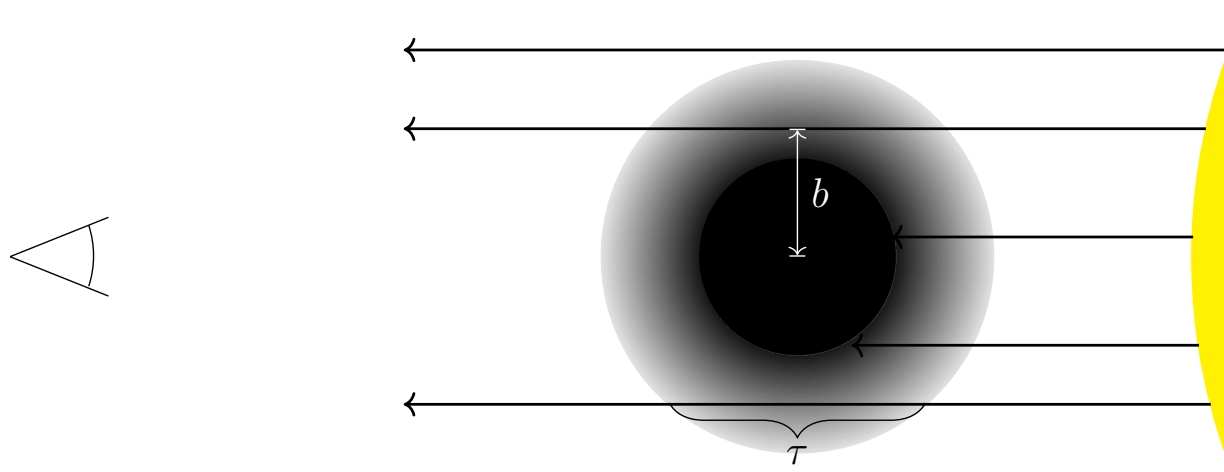

Fig. 1.3. - Illustration of transmission spectrum view from the side. The stellar beams with impact parameter $b$, pointed at the observer in the left, probe the transiting planet's atmosphere along a chord perpendicular to the impact parameter, $b$. The sizes of the planet, star, and orbital separation are not to scale. 
The difference in flux due to total opacity and continuum opacity is then

$$
\Delta F_{\nu} \equiv F_{\nu}-F_{\nu}^{(c)}=\frac{2 \pi I_{\nu}}{d^{2}} \int_{0}^{R_{\star}} b\left(e^{-\tau_{c}(b)-\tau_{l, \nu}(b)}-e^{-\tau_{c}(b)}\right) b \mathrm{~d} b
$$

The contribution from both terms is zero for $b<R_{p}$ due to the continuum opacity, and there is no continuum absorption outside that range, and so this expression can be rewritten

$$
\Delta F_{\nu}=\frac{2 \pi I_{\nu}}{d^{2}} \int_{R_{p}}^{R_{\star}}\left(e^{-\tau_{l, \nu}(b)}-1\right) b \mathrm{~d} b .
$$

Equivalent to the transmission spectrum defined in the observations (e.g., Cauley et al. 2015), the fractional change in flux, relative to the continuum integral at the same frequency, is then

$$
\begin{aligned}
\frac{\Delta F}{F}(\nu) & \equiv \frac{\Delta F_{\nu}}{F_{\nu}^{(c)}} \equiv-\frac{R_{\nu}^{2}-R_{p}^{2}}{R_{\star}^{2}-R_{p}^{2}} \\
& =\frac{2}{R_{\star}^{2}-R_{p}^{2}} \int_{R_{p}}^{R_{\star}}\left(e^{-\tau_{l, \nu}(b)}-1\right) b \mathrm{~d} b .
\end{aligned}
$$

The ratio $\Delta F / F$ will be referred to as the model transmission spectrum (Huang et al. Submitted).

Equation 1.6 also defines the apparent radius of the planet $R_{\nu}$, which is the radius of an opaque disk that can block the same amount of light at given frequency as the planet. The apparent radius is indicated as the dash yellow circle in Figure 1.2. The same effect may also be referred as the radius spectrum, which shows the apparent radius of the planet against wavelength.

From Equation 1.6, one can get an equivalent definition of $R_{\nu}$,

$$
R_{\nu}^{2} \equiv R_{p}^{2}+2 \int_{R_{p}}^{R_{\star}}\left(1-e^{-\tau_{l, \nu}(b)}\right) b \mathrm{~d} b
$$


which can be calculated from theoretical model.

The magnitude of the stellar brightness variation associated with the atmosphere absorption is smaller than the transit depth by a factor of around $2 H / R_{p}$, where $H=$ $k T / \mu g$ is the atmosphere scale height. The absorption of higher ionizing EUV and Xray flux from the host star due to small separation can heat the upper atmosphere of hot Jupiter to a higher temperature and inflates those layers. This ratio of $2 H / R_{p}$ can be $\sim 0.01$ to 0.1 for hot Jupiter. The small brightness variation makes it challenging to determine a transmission spectrum even for hot Jupiters (Burrows 2014a).

Hot Jupiters are subjected to strong ionizing radiation and stellar wind fluxes because of their close distance. The temperature of the upper atmospheres, where the majority of the stellar extreme ultraviolet (EUV) radiation energy is absorbed, increases outward (referred as a "thermal inversion") and can be heated to over 10,000 K (Yelle 2004). This "thermosphere" is analogous to the same region of Earth's upper atmosphere. The scale heights of the dramatically inflated and fully ionized upper atmospheres can approach 5\% of the planetary radius. According to Equation 1.2, these less dense layers can only be optically thick and be observed at frequencies which $\sigma_{\nu}$ are extremely large. Therefore, in the high-resolution transmission spectrum, one can find deep absorption features, created by the upper atmosphere of those close-in planets, at the line core of several atomic resonant lines, such as $\mathrm{H} \mathrm{Ly} \alpha, \mathrm{H} \alpha, \mathrm{Na}$ and $\mathrm{K}$ doublets. In contrast, most of the observational effort has concentrated on the broadband optical/infrared transmission spectrum (e.g., Pont et al. 2013), which probes the lower atmosphere at mbar-bar pressures.

Studies of the optical and near-infrared broadband transmission spectra have revealed that many hot Jupiters have featureless spectra with strong Rayleigh scattering slopes $\left(\lambda^{-4}\right)$ toward short wavelengths, which indicates that the Rayleigh scattering 
by molecules or haze particles dominate the opacity near the planet surface (Pont et al. 2013; Sing et al. 2016). Depending on the species that is responsible for the Rayleigh scattering assumed in the model, the exact pressure of planet extinction surface $R_{p}$ varies from a few $\mu$ bar to hundreds mbar (Lecavelier Des Etangs et al. 2008). With the increase of the altitude, the atmosphere gradually switches from molecule dominated gas near the surface to an atom dominate layer and eventually becomes ionized.

The much weaker than predicted absorption features of water and other molecules in the observed near-infrared spectra has been attributed to the presence of highaltitude clouds and haze layers that effectively obscure absorption features by making the lower atmosphere opaque (Deming et al. 2013; Kreidberg et al. 2014). In these cases, only species that have significant absorption in the uppermost layers of the atmosphere, such as atomic resonant lines, can be observed.

As the hot gas in the upper thermosphere is more weakly bound to the planet, it's been shown both theoretically (e.g., Yelle 2004; Murray-Clay et al. 2009) and observationally (e.g., Vidal-Madjar et al. 2003; Lecavelier Des Etangs et al. 2010) that the heated gas in this upper atmosphere can escape from the planet. Since the first discoveries of hot Jupiter, the evaporation of their atmosphere has been of interest. Furthermore, Miguel et al. (2015) showed that the Ly $\alpha$ radiation changes atmospheric chemistry in the molecular layer significantly, especially the photolysis of water. Thus, a model of the upper atmosphere is required to determine the incoming radiation and set the upper boundary condition in the study of lower part of the planetary atmosphere. Therefore, the atomic resonance lines in the hot Jupiter transmission spectrum are an important probe to understand these exotic worlds. 


\subsection{Observations of the Upper Atmosphere of HD $189733 b$}

The hot Jupiter HD 189733b was discovered orbiting the star HD 189733A using the transit method by Bouchy et al. (2005). HD 189733A is a K2V main-sequence star, with a red dwarf companion at a distance over $200 \mathrm{AU}$. The orbital and physical parameters of HD 189733 and HD 189733b are listed in Table 1.1. Being the closest transiting hot Jupiter to Earth, HD 189733b is a subject for extensive atmospheric examination, through high- and low-resolution instruments, both from ground and space.

The first HD 189733b atmosphere detection was claimed by Redfield et al. (2008) using the Hobby-Eberly Telescope (HET) High Resolution Spectrograph (HRS), which detects the NaD absorption by the atmosphere. This is also the first ground-based detection of an exoplanetary atmosphere.

Most of the spectra-photometric measurements of exoplanet atmospheres have been performed through space-based observations. Because of the added compli-

Table 1.1: Adopted values for the orbital and physical parameters of HD 189733 and HD $189733 \mathrm{~b}$

\begin{tabular}{cc}
\hline \hline Star mass & $M_{\star}=1.60 \times 10^{33} \mathrm{~g}$ \\
Star radius & $R_{\star}=5.60 \times 10^{10} \mathrm{~cm}$ \\
Star distance & $d=19.45 \mathrm{pc}$ \\
Semi-major axis & $a=0.031 \mathrm{AU}$ \\
Planet mass & $M_{p}=2.17 \times 10^{30} \mathrm{~g}$ \\
Planet radius & $R_{p}=8.137 \times 10^{9} \mathrm{~cm}$ \\
Planet surface gravity & $g_{p}=G M_{p} / R_{p}^{2}=2.2 \times 10^{3} \mathrm{~cm} \mathrm{~s}^{-2}$ \\
\hline
\end{tabular}

Note. - Source: exoplanets.org 
cations posed by the Earth's atmosphere, ground-based observations cannot obtain absolute photometry without a reference star. To solve this problem, instead of the absolute flux $F_{\nu}$ or $F_{\nu}^{(o u t)} / F_{\nu}^{(i n)}$, a normalized transit spectra $\Delta F / F$ is considered (see Equation 1.1 and 1.6), which removes the transit depth due to the planet's disk and only allows relative measurement of a planet's atmospheric absorption (Redfield et al. 2008). Although ground-based telescopes have much larger systematic errors and require a telluric subtraction, a larger aperture, high-resolution spectroscopy, and observing multiple transits can provide a higher spectral resolution transmission spectrum and make a valuable contribution to the understanding of the planetary upper atmosphere.

Following the first detection, the $\mathrm{Na}$ transmission spectrum has been observed many times with different strategies. Based on the result of Redfield et al. (2008), Jensen et al. (2011) made more observations with the same instrument. Using over 200 spectra taken both in transit and out of transit performed across transits in two years, they measured a $(5.26 \pm 1.69) \times 10^{-4}$ absorption depth with a $12 \AA$ bandwidth, or a maximum $\sim 0.9 \%$ absorption depth with $0.2 \AA$ bin width, caused by the planetary atmosphere only.

Czesla et al. (2015), Cauley et al. (2016), and Barnes et al. (2016) confirmed the Na detection. Because Czesla et al. (2015) and Barnes et al. (2016) didn't make a transmission spectrum, and large positive peaks appear in the $\mathrm{NaD}$ transmission spectra of Redfield et al. (2008), Jensen et al. (2011), and Cauley et al. (2016), which is unphysical, these $\mathrm{Na}$ observations are not included in this study.

Huitson et al. (2012) observed seven transits using Space Telescope Imaging Spectrograph (STIS) G750M grating onboard the Hubble Space Telescope (HST) covering a wavelength range of $5808-6380 \AA$, and three of these transits collected data of 
high quality. The obtained high signal-to-noise ratio $(\mathrm{S} / \mathrm{N})$ medium-resolution NaD transmission spectra, shown as the red curve in the Figure 1.4. They constructed a vertical temperature profile of the upper atmosphere based on the measured the spectral profile, which shows the temperature rises with altitude.

Wyttenbach et al. (2015) analyzed the archival spectra of three transits observed by High-Accuracy Radial-velocity Planet Searcher (HARPS) echelle spectrograph on the ESO $3.6 \mathrm{~m}$ telescope. The very high spectral resolution $(R \sim 115000)$ covers the wavelength region between $380 \mathrm{~nm}$ and $690 \mathrm{~nm}$. Shown as the black crosses in Figure 1.4, the reduced NaD transmission spectrum has high resolution and $\mathrm{S} / \mathrm{N}$. The maximum absorption depth with $0.2 \AA$ bin width is about $0.95 \%$, consistent with the result in Jensen et al. (2011). The resolved Na line core shows that the atmosphere temperature keeps increasing above the atmosphere layer studied by Huitson et al. (2012).

Besides $\mathrm{NaD}, \mathrm{H} \alpha$ is another strong resonant line in the optical band, which is possible to observe with ground-based telescopes. Among detected transitions, $\mathrm{H} \alpha$ is a sensitive probe of the planet's upper atmosphere because the excitation and deexcitation processes for $\mathrm{H}(2 \ell)$, the absorber of $\mathrm{H} \alpha$, are strongly dependent on the local particle densities, temperature, and radiation field. Fortney et al. (2010) modeled the HD 189733b transmission spectrum assuming thermal chemical equilibrium. They did not predict any $\mathrm{H} \alpha$ absorption because hydrogen can hardly be excited in chemical equilibrium at the temperature of the atmosphere.

Jensen et al. (2012) reduced the H $\alpha$ section of the HET/HRS transmission spectra (Jensen et al. 2011), and showed a maximum $\sim 2 \%$ transit depth caused by the planetary atmosphere. As followup, using the High Resolution Echelle Spectrometer (HiRES) on Keck I, Cauley et al. (2015) and Cauley et al. (2016) obtained the H $\alpha$ 


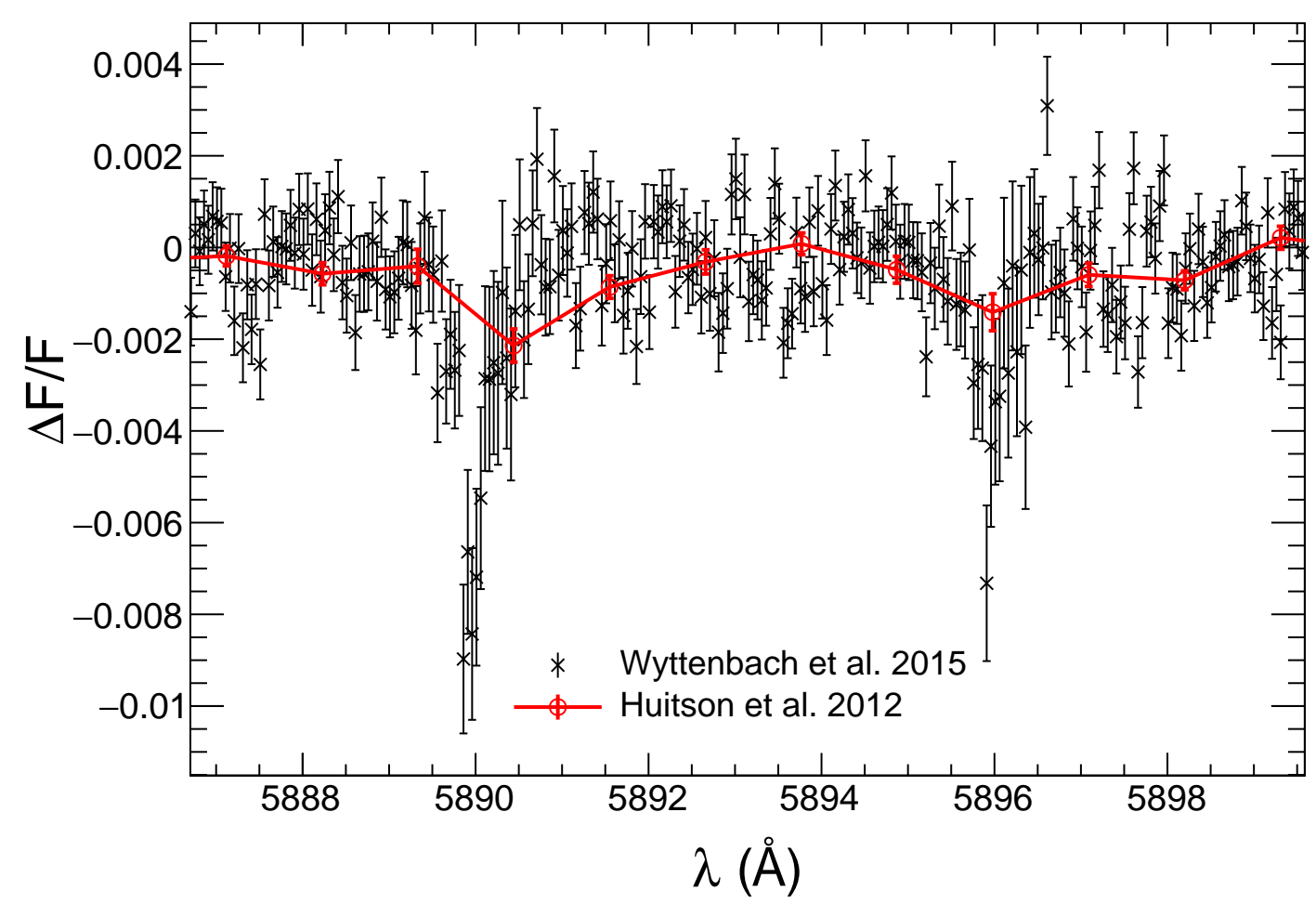

Fig. 1.4. - Observed NaD doublet transmission spectra by Huitson et al. (2012) (in red) and by Wyttenbach et al. (2015) (in black). A $5 \times$ binning and $10 \mathrm{~km} \mathrm{~s}^{-1}$ shift to the red have applied to the spectrum of Wyttenbach et al. (2015), as suggested in their paper.

transmission spectra of three more transits, shown in Figure 1.5. Cauley et al. (2015) measured a maximum transit depth $\sim 0.9 \%$ by the planetary atmosphere, and Cauley et al. (2016) measured $\sim 1.7 \%$.

Both the $\mathrm{H} \alpha$ transmission spectrum observed by Cauley et al. $(2015,2016)$ and the NaD transmission spectrum presented by Wyttenbach et al. (2015) have the spectral resolution to resolve the line core. The similar line center transit depths may indicate that the line core absorption features of both species are mostly contributed by the same region in the atmosphere.

$\mathrm{H} \beta$ and $\mathrm{H} \gamma$ transmission spectra were also reported by Cauley et al. (2015, 2016). 
The results of Jensen et al. (2012) are not included in the study since these observations were not performed across a single transit.

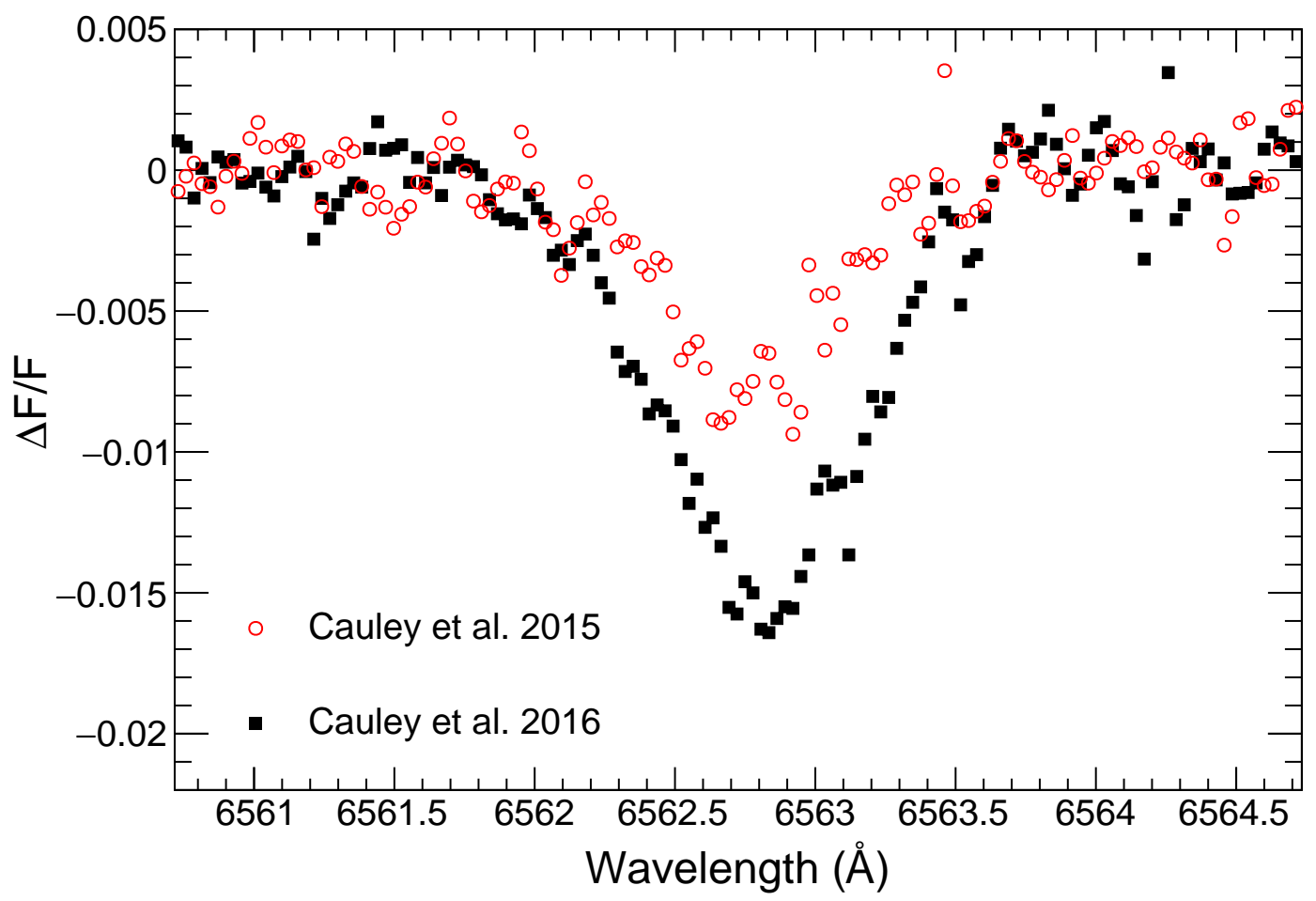

Fig. 1.5.- Observed $\mathrm{H} \alpha$ transmission spectra by Cauley et al. (2015) (in red open circle) and by Cauley et al. (2016) (in black filled square).

Barnes et al. (2016) used archival HARPS data to measure in-transit H $\alpha$ transmission spectra. Based on the velocity maps of the absorption signatures, they concluded that the transit signatures measured in the chromospherically sensitive lines, in particular $\mathrm{H} \alpha$ and the $\mathrm{Ca}$ II $\mathrm{H}$ and $\mathrm{K}$ lines, are the result of the contrast effect of stellar active regions, rather than absorption by planetary material. Cauley et al. (2017) reanalyzed the seven full $\mathrm{H} \alpha$ transit observations of HARPS and HiRES, and find that the transit signal is unlikely to be caused by the stellar active region alone. The planet must consistently transit active latitudes with very strong facular and plage emission regions in order to reproduce the observed line strengths. 
The abundant ground state neutral hydrogen, Ly $\alpha$ has been an important tool in probing the planetary upper atmosphere. Transits can provide important clues toward understanding mass loss from exoplanets, which can carry a significant amount of gas to a few planetary radii. Although the transit signal near the Ly $\alpha$ line center is strongly contaminated by the interstellar hydrogen absorption and geocoronal emission, the atmosphere absorption can generate an observable decrease of flux in the Ly $\alpha$ line wing. In the Ly $\alpha$ transmission spectrum of another hot Jupiter HD 209458b, Vidal-Madjar et al. (2003) detected a large transit depth at a high velocity in the line wing. This absorption was interpreted as the neutral hydrogen in the planetary upper atmosphere, the $\operatorname{Ly} \alpha$ absorber, which is escaping at a very high velocity. This was the first claim of detection of atmospheric escape from an exoplanet.

The host star HD 189733 emits one of the highest Ly $\alpha$ flux ever measured for transit observations except for 55 Cnc (Ehrenreich et al. 2012), which makes HD 189733b a particularly good candidate to study atmospheric evaporation. Lecavelier Des Etangs et al. (2010) made a 3.5 $\sigma$ planetary atmosphere Ly $\alpha$ absorption detection by lowresolution spectrograph using the Advanced Camera for Surveys (ACS) instrument onboard the HST and integrated over the entire Ly $\alpha$ feature.

Lecavelier des Etangs et al. (2012) observed two transits on 6 April 2010 and 7 September 2011 with the higher resolution STIS G140M grating onboard the HST. Disappointingly, no significant Ly $\alpha$ atmosphere absorption detected in the first transit. While in contrast, an excess absorption by the atmosphere was detected in the second observation with a level consistent with Lecavelier Des Etangs et al. (2010). Bourrier et al. (2013) reanalyzed the second transit and showed a $12.3 \pm 3.7 \%(3.3 \sigma)$ total absorption depth (including the planetary disk occultation) due to the atmospheric hydrogen on the blue wing, and a $5.5 \pm 2.7 \%(2.0 \sigma)$ absorption on the red 
wing. This may imply a strong temporal variation in the physical condition of the planetary upper atmosphere.

Contemporaneous observations of the second transit in X-ray $(0.3-3 \mathrm{keV})$ was made with the X-ray telescope (XRT) of the Swift spacecraft. They measured an averaged X-ray flux of $3.6 \times 10^{-13} \mathrm{ergs}^{-1} \mathrm{~cm}^{-2}$ in the energy band. The observation accuracy is not enough to detect the planet transit in the X-ray, but a significant stellar X-ray variability was shown. Most notably, a stellar flare event with tripled X-ray flux has been detected.

To reach a much better accuracy, Poppenhaeger et al. (2013) observed a series of X-ray transits using Chandra ACIS-S. Due to the very large absorption cross section of metals at X-ray wavelength, the atmosphere becomes optically thick with a relatively small amount of metal atoms. Because the stellar flux at this wavelength is highly concentrated in the active regions and vary strongly with time, the transit light curves are unpredictable and do not at all resemble the "canonical" photospheric transit light curves. Therefore, although the transit depth of a hot Jupiter may be large at X-ray wavelengths, one would have severe difficulties in the interpretation of the resulting light curve, for any given single transit light curve (Schmitt 2017).

Poppenhaeger et al. (2013) detected a 6\% - 8\% X-ray total transit depth, which is substantially larger than the $2.41 \%$ broadband optical transit depth. If confirmed, this result indicates that a considerable amount of metal atoms exist up to a height at least 0.5 planet radius above the planet surface. So far, this is the only atmosphere transit detection of any exoplanetary system in X-rays. Additional observations will be necessary to fully exclude the possibility that coronal inhomogeneities influence the result.

In addition, a detection of O I 1306 has been made by Ben-Jaffel \& Ballester 
(2013). Pont et al. (2008, 2013) claimed a trace of potassium absorption using HST/ACS, which is in contrast to the findings of Jensen et al. (2011) who did not detect potassium using high-resolution spectroscopy. Cauley et al. (2016) measured a marginal amount of Mg I 5184. Barnes et al. (2016) and Czesla et al. (2015) also claimed a possible detection of Ca II H \& $\mathrm{K}$ lines.

\subsection{Project Overview}

Despite numerous observations, the only previous models of the $\mathrm{H} \alpha$ transmission spectrum was by Christie et al. (2013), who constructed a hydrostatic atmosphere model. In that work, a detailed treatment of Ly $\alpha$ radiation transfer was not included, and hence the role of Ly $\alpha$ excitation deep in the atmosphere was not appreciated. Christie et al. (2013) showed that if collisional excitation dominates, it would lead to a fairly constant $\mathrm{H}(2 \mathrm{~s})$ density within the atomic layer, because of the combination of increasing temperature and decreasing $\mathrm{H}(1 \mathrm{~s})$ density with radius. In attempting to improve on their model, it was found that Ly $\alpha$, especially from recombinations occurring within the atmosphere, could give a radiative excitation rate to $\mathrm{H}(2 \mathrm{p})$ much larger than the collisional excitation rate, and that this excitation could occur deeper in the atmosphere where the $\mathrm{H}(1 \mathrm{~s})$ density is higher, even though the temperature is much lower there. This key insight motivated the detailed Ly $\alpha$ radiative transfer treatment in the present work.

As mentioned in the section 1.2, the line core absorption features of HD 189733b $\mathrm{H} \alpha$ and $\mathrm{NaD}$ may be mostly contributed by the same region in the atmosphere. This fact provides an extra constraint on the atmosphere model. And an atmosphere model of this region that can explain both absorption features can largely improve the knowledge about exoplanet upper atmosphere. 
Since the temperature of the molecular layer is below $3000 \mathrm{~K}$, and the molecular hydrogen has a large absorption cross section to Ly $\alpha$ photon (Black \& van Dishoeck 1987), both the collisional excitation rate and radiative excitation rate are too small to create enough $\mathrm{H}(2 \ell)$ to absorb the $\mathrm{H} \alpha$ in the molecular layer. These results suggest that the absorption features of both $\mathrm{H} \alpha$ and $\mathrm{Na}$ near line center are tracing the atomic layer in the HD 189733b atmosphere.

As this work shows, the temperature, and hence scale height, in the region optically thick to $\mathrm{H} \alpha$ is (roughly) set by a balance of photoelectric heating and line cooling by metal species, mainly $\mathrm{Mg}$ I and $\mathrm{Na}$ I. If only photoelectric heating and line cooling from hydrogen were included, the atmosphere would be hotter by $\simeq 2000-3000 \mathrm{~K}$ (Christie et al. 2013), giving transit depths far too large in comparison to observations. Furthermore, several studies (García Muñoz 2007; Koskinen et al. 2013a; Lavvas et al. 2014) suggested that the transition from atomic to molecule hydrogen occurs at pressures $P \simeq 10 \mu$ bar. These studies included detailed heating and cooling physics in the molecular layer. But transmission spectra for the Na D doublet and Mg lines may in principle provide further constraints on atmosphere models around this transition altitude. In addition, the atmospheric temperature of HD 189733b derived from the Na doublet transmission spectrum by Huitson et al. (2012) and Wyttenbach et al. (2015) is significantly lower than the modeled upper atmosphere temperature found in Salz et al. (2016) and Christie et al. (2013). A model of the Na transmission spectrum is required to understand these contradictory results.

Based on the path of Christie et al. (2013), in this work, a detailed one-dimensional hydrostatic atmosphere is constructed over the pressure range $P=10^{-4}-10 \mu \mathrm{bar}$, encompassing the region dominated by atomic hydrogen. The atomic hydrogen level population is computed including both collisional and radiative transition rates. Ion- 
ization equilibrium and balance of heating and cooling processes are enforced at each level of the atmosphere. Metal species with solar abundance are included. Photoelectric heating is included using a synthetic spectrum for HD 189733, and secondary electron ionization and excitation are included. The Ly $\alpha$ radiation intensity is computed using a Monte-Carlo code which includes resonant scattering, as well as photon destruction by true absorption, photoionization from the $n=2$ state, and collisional de-excitation. Both the incident stellar Ly $\alpha$ and internal sources due to recombination cascade and collisional excitation are included. The transmission spectrum of $\mathrm{H} \alpha$ and $\mathrm{NaD}$ are calculated based on the simulated atmosphere model. The dependence of the transit profile on Lyman continuum (LyC) and metal abundances are considered. The discrepancy in the upper atmosphere temperature between theoretical and observational is investigated. 


\section{Chapter 2}

\section{$\mathrm{H} \alpha$ and $\mathrm{NaD}$ Transmission Spectrum of HD 189733b}

This chapter is based on the submitted paper, Huang et al. (Submitted).

\subsection{Analytic Estimates}

Observations of the $\mathrm{H} \alpha$ transmission spectrum for HD 189733b show a $\sim 1 \%$ line center transit depth, and a half-width of $\sim 0.4 \AA$ (e.g. Cauley et al. (2015)). Wyttenbach et al. (2015) measured a nearly $\sim 1 \%$ transit depth for both NaD lines, among which the Na I $5890 \AA$ is slightly deeper. This section contains analytic estimates for the conditions in the atmosphere required to generate the observed $\mathrm{H} \alpha$ and $\mathrm{NaD}$ absorption lines.

The outermost reaches of HD 189733b's atmosphere are highly ionized by stellar photons. Moving inward, the radiative recombination rate eventually increases to the point that the atmosphere is dominated by atomic hydrogen, at a pressure level $P \simeq$

$10^{-3} \mu$ bar. The temperature near the transition from ionized to atomic is regulated 
to be near $T \simeq 10,000 \mathrm{~K}$, which is far too hot for molecular hydrogen to form, and hence there must be a layer of atomic hydrogen extending over the temperature range $T \simeq 2,500-10,000 \mathrm{~K}$. In the hydrostatic model presented in this paper, the temperature in the atomic layer is set by a balance of photoelectric heating and atomic line cooling, for which $\mathrm{H}_{2}$ will dominate at $P \gtrsim 10 \mu$ bar. In terms of size, the atomic hydrogen layer extends over $\sim 10$ pressure scale heights, has a mean molecular weight $\mu \simeq 1.3$ and mean temperature $T \simeq 5,000 \mathrm{~K}$. As compared to the underlying molecular layer, with mean molecular weight $\mu \simeq 2.3$ and temperature $T \simeq 1,000 \mathrm{~K}$, the scale height in the atomic layer is larger by a factor of $\sim 10$ compared to the molecular layer, and hence can give rise to absorption to much larger altitudes.

The origin of the $\mathrm{H}(2 \ell)$ population requires a detailed level population calculation. In the present model of the atomic layer, it is found that radiative excitation by Ly $\alpha$ creates a nearly constant $\mathrm{H}(2 \ell)$ density over $\sim 6$ pressure scale heights near the base of the atomic layer. This is the cause of the $\mathrm{H} \alpha$ absorption. The underlying molecular layer is expected to be optically thin to $\mathrm{H} \alpha$ for two reasons. First, the density of atomic hydrogen drops rapidly into the molecular layer (e.g. Lavvas et al. 2014), as compared to the base of the atomic layer, due to the much lower temperature there. Second, the mean free path to true absorption of $\mathrm{Ly} \alpha$ by $\mathrm{H}_{2}$ (Black \& van Dishoeck 1987) rapidly decreases as the $\mathrm{H}_{2}$ density increases, and hence the Ly $\alpha$ intensity is expected to drop rapidly in the molecular layer, with an associated decrease in excitation to the $\mathrm{n}=2$ state.

The measured line center transit depth $\Delta F / F \sim 1 \%$ requires a certain area to be optically thick. The scale height in the atomic layer is

$$
H=\frac{k_{B} T}{\mu m_{p} g}=1500 \mathrm{~km}\left(\frac{T}{5000 \mathrm{~K}}\right),
$$


where the mean molecular weight has been assumed to be $\mu=1.3$. If $\mathrm{N}$ scale heights are optically thick, as compared to the neighboring continuum radiation, this gives an extra absorption depth

$$
\begin{aligned}
\frac{\Delta F}{F} & \simeq N \frac{2 \pi R_{p} H}{\pi R_{\star}^{2}}=8 \times 10^{-4} N\left(\frac{T}{5000 \mathrm{~K}}\right) \\
& =0.5 \%\left(\frac{N}{6}\right)\left(\frac{T}{5000 \mathrm{~K}}\right) .
\end{aligned}
$$

Hence the measured line center depth can only be explained by a layer extending many pressure scale heights, and with high temperature $T \gtrsim 5,000 \mathrm{~K}$.

The line center optical depth must be greater than unity over the above annulus. As will be shown, $n_{2 \ell}$ is nearly constant over a large pressure range. Then for an effective path length $2 \sqrt{2 N R_{p} H} \simeq 7 \times 10^{9} \mathrm{~cm}(N / 6)^{1 / 2}(T / 5,000 \mathrm{~K})^{1 / 2}$ and $\mathrm{H} \alpha$ line center cross section $\sigma_{0}=5 \times 10^{-13} \mathrm{~cm}^{2}(5,000 \mathrm{~K} / \mathrm{T})^{1 / 2}$, the maximum line center optical depth is

$$
\tau_{0} \simeq 35 \times\left(\frac{n_{2 \ell}}{10^{4} \mathrm{~cm}^{-3}}\right)
$$

for the fiducial value $n_{2 \ell}=10^{4} \mathrm{~cm}^{-3}$.

The line width is mainly set by the temperature and the maximum line center optical depth. For sightlines optically thick to $\mathrm{H} \alpha$ at a line center, the optical depth at distance $x=\left(\nu-\nu_{0}\right) / \Delta \nu_{D}$ from line center is $\tau(x)=\tau_{0} \exp \left(-x^{2}\right)$, when Doppler broadening dominates. All frequencies out to $x \simeq \sqrt{\ln \left(\tau_{0}\right)}$ are then optically thick. In velocity units, the line width is then

$$
\Delta v=\left(\frac{2 k_{B} T}{m_{p}}\right)^{1 / 2}\left(\ln \tau_{0}\right)^{1 / 2}=9.1 \mathrm{~km} \mathrm{~s}^{-1}\left(\ln \tau_{0}\right)^{1 / 2}
$$


or in wavelength units

$$
\Delta \lambda=0.2 \AA\left(\ln \tau_{0}\right)^{1 / 2} .
$$

Since $\tau_{0} \gg 1$ for $\mathrm{H} \alpha$ over a large region, the width will be larger than the thermal width.

If Ly $\alpha$ excitation is balanced by radiative de-excitation, and $\ell$-mixing populates the $2 \mathrm{~s}$ state, the abundances relative to the ground state are

$$
\begin{aligned}
\frac{n_{2 p}}{n_{1 s}} & \simeq 3 \frac{n_{2 s}}{n_{1 s}}=\frac{J_{12} B_{12}}{A_{21}} \\
& \simeq 10^{-8}\left(\frac{J_{12}}{10^{-9} \mathrm{erg} \mathrm{cm} \mathrm{cm}^{-2} \mathrm{~s}^{-1} \mathrm{~Hz}^{-1}}\right)
\end{aligned}
$$

where $A_{21}$ and $B_{12}$ are the Einstein $\mathrm{A}$ and $\mathrm{B}$ (absorption) coefficient respectively, following the definition in Rybicki \& Lightman (1979). The peak Ly $\alpha$ intensity $J_{12} \simeq$ $0.1 F_{\mathrm{LyC}} / \Delta \nu_{D} \simeq 10^{-8} \mathrm{erg} \mathrm{cm}^{-2} \mathrm{~s}^{-1} \mathrm{~Hz}^{-1}$ is found near the peak in photoionization of $\mathrm{H}(1 s)$ near $P \simeq 10^{-3} \mu$ bar. Here, $F_{\mathrm{LyC}} \simeq 10^{4} \mathrm{erg} \mathrm{cm}^{-2} \mathrm{~s}^{-1}$ is the LyC flux deposited in that region, and it is assumed that each ionization is balanced by a recombination producing a Ly $\alpha$ photon. While the ratio of excited state to ground state is high near the peak in $\operatorname{Ly} \alpha$, the $\mathrm{H}(1 \mathrm{~s})$ density there is too small for significant $\mathrm{H}(2 \ell)$ density.

The key question for the $\mathrm{H}(2 \ell)$ population is how fast the Ly $\alpha$ intensity decreases moving deeper into the atmosphere. If $\operatorname{Ly} \alpha$ intensity does not drop off too fast, the rapid increase in $\mathrm{H}(1 \mathrm{~s})$ density with depth will lead to higher $\mathrm{H}(2 \ell)$ density deeper in the atmosphere. One can imagine two limits to answer this question. In the first limit, there is a shallow source of Ly $\alpha$ at optical depth $\tau_{s}$ and the intensity $J_{\nu}(x, \tau)$ is desired at $\tau \gg \tau_{s}$. An analytic solution based on the Fokker-Planck equation given 
in Harrington (1973) is

$$
J_{\nu}(x, \tau) \simeq 0.1\left(\frac{F_{0}}{\Delta \nu_{D}}\right)\left(\frac{\tau_{s}}{\tau}\right)
$$

This expression is valid in the plateau of the intensity near line center. Since Ly $\alpha$ optical depth $\tau \propto n_{1 s}$, this scaling for $J_{\nu}$ would give $n_{2 \ell} \propto n_{1 s} J_{\nu} \simeq$ constant with depth. The second limit to imagine is where radiation is emitted and absorbed locally, which is appropriate deep in the atmosphere where $\tau \sim 10^{8}$ scatterings are required to escape the atmosphere. For a constant, frequency-integrated source function $S$, and true absorption by metal species, the frequency-integrated photon energy equation becomes $S \simeq n_{m} \sigma_{m} J$, where $J$ is the frequency-integrated intensity, $n_{m}$ is the metal number density and $\sigma_{m}$ is the metal photoionization cross section at Ly $\alpha$. For a constant mixing ratio, $n_{m} \propto n_{1 s}$, and again $n_{2 \ell} \simeq$ constant. While the scaling found by these two estimates, constant $2 \ell$ density, is the same, it is found that the local balance of sources and sinks is the applicable limit in the present atmosphere model.

Up to this point, the estimates have been concerned with the $\mathrm{H} \alpha$ transmission line, however, the center of each line in the $\mathrm{Na}$ doublet may also be formed in the atomic layer. Because the cross section of Na I 5890 is larger than Na I 5896 by a factor of 2, the difference in transit radius between the two resolved line centers corresponds to $\ln (2) H$, assuming a constant Na I number fraction. Keeping in mind the error bars in the measurement, according to Wyttenbach et al. (2015), the difference in transit radius between the $\mathrm{NaD}$ lines is $\sim 3000 \mathrm{~km}$, which gives a local scale height $H \simeq$ $4300 \mathrm{~km}$. Plugging in the transit radius $R(\mathrm{Na})=9.4 \times 10^{9} \mathrm{~cm}$ to compute the gravity, assuming $\mu=1.3$, the temperature derived from the line centers is $T \simeq 11,000 \mathrm{~K}$. In order to explain this scale height with a molecular gas, the temperature has to be higher by a factor of 2 . But this high temperature is inconsistent with the gas being 
in molecular form.

\section{$2.2 \quad$ Hydrogen Level Population}

Balmer line photons are absorbed by the $2 \ell$ excited states of $\mathrm{H}$. Due to ionization and the subsequent recombination cascades, and a radiation excitation temperature different from the gas temperature, the level populations are not set by the Boltzmann distribution at the local gas temperature. Therefore, a study of the $\mathrm{H}$ level population over the range of densities, temperatures, and intensities found in hot Jupiter atmosphere is required. The following processes are considered.

1. Hydrogen radiative (de-)excitation of all possible electric dipole transitions between multiplets up to $n=6$ (Wiese \& Fuhr 2009);

2. Electron collisional (de-)excitation for transitions from $1 s$ to each sub-state $\ell$ up to $n=5$, from $2 s$ to each sub-state $\ell$ up to $\mathrm{n}=5$, and from $2 p$ to each sub-state $\ell$ up to n=3 (CHIANTI database, Dere et al. (1997); Del Zanna et al. (2015));

3. Electron collisional $\ell$-mixing between $2 s$ and $2 p$ (Seaton 1955);

4. Proton collisional $\ell$-mixing between $2 s$ and $2 p$ (Seaton 1955), and $\ell$-mixing for levels $3 \leq n \leq 6$ (Vrinceanu et al. 2012);

5. Electron collisional ionization and three-body recombination for each sub-state $\ell$ up to $\mathrm{n}=4$ and total ( $\ell$-unresolved) rates for $n=5$ and 6 (Janev et al. 2003). The cross sections are assumed to be equal for all sub-states in the same level for $n=5$ and 6 ;

6. Photoionization of each sub-state $\ell$ up to $n=6$ (Team 1995). The corresponding recombination rate can be calculated using the Milne relation. 


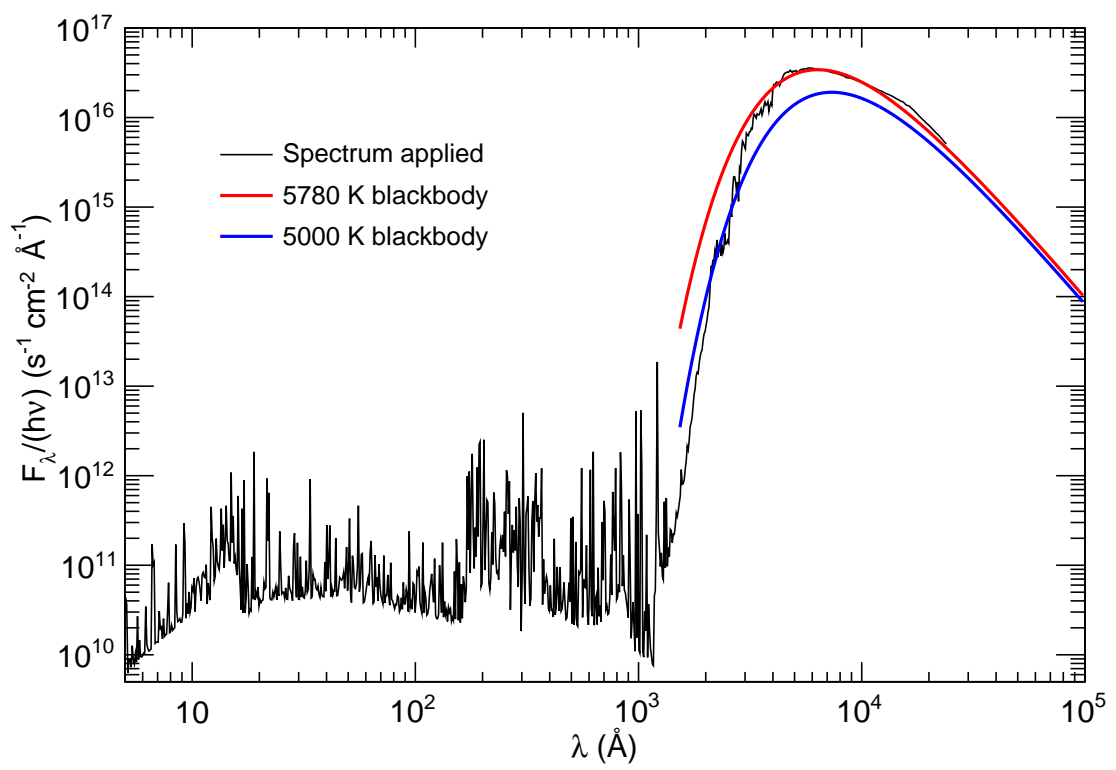

Fig. 2.1. - Stellar photon number flux spectrum $\left(F_{\lambda} /(h \nu)\right)$ of HD 189733 against wavelength $(\lambda)$ used in the model at distance of HD 189733b. Blackbody spectra at two temperatures are shown for comparison.

The employed stellar flux $\left(F_{\lambda}\right.$, see Figure 2.1) is a combination of the synthetic spectrum for $\lambda<1200 \AA$ (X-exoplanets Archive at the CAB, Sanz-Forcada et al. 2011) and the quiet solar spectrum for $\lambda \geq 1200 \AA$ (LASP Interactive Solar Irradiance Data Center, Lindholm et al. 2011). It is normalized to the distance $a=0.031 \mathrm{AU}$ of HD 189733b from its parent star. The stellar flux attenuation due to bound-bound transitions is not included in this calculation because it is relatively unimportant in setting the level populations, and performing radiation transfer calculations in addition to Ly $\alpha$ would greatly complicate the analysis. Bound-bound transitions due to $\operatorname{Ly} \beta$, Ly $\gamma$ etc. are ignored as it is assumed that these photons rapidly down-convert into a Ly $\alpha$ photon and lower series photons at the large optical depths of interest for these lines. 
The level population is determined by the kinetic equilibrium between production and loss processes. The equation of rate equilibrium for the state $j$ is

$$
\begin{aligned}
& \alpha_{j} n_{e} n_{p}+\beta_{j} n_{e}^{2} n_{p}+\sum_{k} C_{k \rightarrow j}^{(e)} n_{e} n_{k}+\sum_{k} C_{k \rightarrow j}^{(p)} n_{p} n_{k}+\sum_{k>j} A_{k \rightarrow j} n_{k}+\sum_{k<j} B_{k \rightarrow j} \bar{J}_{k \rightarrow j} n_{k} \\
& =\left(\Gamma_{j}+\sum_{k} C_{j \rightarrow k}^{(e)} n_{e}+\sum_{k} C_{j \rightarrow k}^{(p)} n_{p}+C_{j \rightarrow \infty}^{(e)} n_{e}+\sum_{k>j} B_{j \rightarrow k} \bar{J}_{j \rightarrow k}+\sum_{k<j} A_{j \rightarrow k}\right) n_{j},
\end{aligned}
$$

where $\alpha_{j}$ and $\beta_{j}$ denote radiative and three-body recombination rate coefficients for state $j$, respectively. Case $\mathrm{B}$ recombination with $\alpha_{1 s} \equiv 0$ is employed. The $n_{k}$ is the number density of hydrogen in sub-state $k$. The inequality $k>j$ denotes a downward transition from a state with principle quantum number of state $k$ larger than that of state $j$. The rate coefficients $C_{j \rightarrow k}^{(e / p)}$ are for electron/proton impacts causing a transition from state $j$ to state $k$, and the state $\infty$ represents ionization. The rate for proton collisions is only included for the $\ell$-mixing transitions at fixed principal quantum number. The spontaneous radiative decay rates are $A_{j \rightarrow k}$. The photoionization rate from state $j$ is denoted $\Gamma_{j}$, and attenuation from the overlying gas is included in the ground state photoionization calculation. The optical depth for ionizing photons is computed as

$$
\tau_{\text {att }, \nu}=\sigma_{1 s}(\nu) N_{1 s}+\sum_{m} \sigma_{m}(\nu) N_{m}
$$

where $N_{k}(r)=\int_{r}^{\infty} \mathrm{d} r^{\prime} n_{k}\left(r^{\prime}\right)$ is the column of species $k$ above the layer under consideration. The sum over the subscript $m$ stands for all metal species considered. As discussed in Section 2.3, this is $m=$ neutral and first ionized C, O, Mg, Si, and S, and neutral $\mathrm{Na}$ and $\mathrm{K}$. Including the attenuation factor $e^{-\tau_{\mathrm{att}}, \nu}$, the photoionization rate 
is

$$
\Gamma_{j, \mathrm{pi}}=\int_{\nu_{j, \mathrm{th}}}^{\infty} \sigma_{j, \mathrm{pi}}(\nu) \frac{F_{\nu}}{h \nu} e^{-\tau_{\mathrm{att}, \nu} \mathrm{d} \nu}
$$

where $\nu_{\text {th }}$ is the corresponding photoionization threshold frequency. The photoionization rates and photoelectric heating rates (see Equation 2.28 and 2.31) at $\tau_{\text {att }}=0$ are listed in Table 2.1.

The H(1s) state can also undergo "secondary ionization" by photoelectrons generated when a photon with much higher energy than the ionization threshold ionizes a hydrogen or metal atom. If $n_{e} / n_{1 s}$ is small, photoelectrons can cause $\mathrm{H}(1 \mathrm{~s})$ collisional ionization and excitation before sharing their energy with other electrons through Coulomb collisions, which would increase the photoionization rate and reduce the photoelectron heating efficiency. Different from the treatment of constant efficiency applied in Yelle (2004) and Murray-Clay et al. (2009), or the ionization fraction $x_{e}=n_{e} / n_{1 s}$ independent efficiency applied in Koskinen et al. (2013a), an efficiency dependent on local $x_{e}$ and photoelectron energy $E=h\left(\nu-\nu_{\text {th }}\right)$ is used here. Given the incoming photon frequency $\nu$ and ionization threshold energy $E_{\mathrm{th}}$, Draine (2011) gives the number of secondary ionizations per ionizing photon, in the case of $E>50 \mathrm{eV}$ and $x_{e}<1.2$, to be

$$
k_{e}(E)=\left(\frac{E-15 \mathrm{eV}}{35 \mathrm{eV}}\right)\left(\frac{1-x_{e} / 1.2}{1+18 x_{e}^{0.8} / \ln (E / 35 \mathrm{eV})}\right) .
$$

After correcting for the secondary ionizations, the form for the $\mathrm{H}(1 \mathrm{~s})$ ionization rate which is used in the rate equations becomes

$$
\begin{aligned}
\Gamma_{1 s} & =\Gamma_{1 s}^{1 \mathrm{st}}+\Gamma_{1 s}^{2 \mathrm{nd}} \\
& =\int_{\nu_{\mathrm{th}}}^{\infty} \sigma_{1 s}(\nu)\left(1+k_{e}\right) \frac{F_{\nu}}{h \nu} e^{-\tau_{\mathrm{att}, \nu}} \mathrm{d} \nu
\end{aligned}
$$


where $\Gamma_{1 s}^{1 s t}$ and $\Gamma_{1 s}^{2 n d}$ stand for primary and secondary photoionization rate from $\mathrm{H}(1 \mathrm{~s})$ respectively. Secondary photoionization becomes an important consideration at pressures $P \gtrsim 0.1 \mu$ bar, where the initially more abundant lower energy photons have already been absorbed higher in the atmosphere, and the dominant photons being absorbed can induce at least one secondary ionization on average.

Lastly, the bound-bound radiative excitation rates are given by

$$
B_{l \rightarrow u} \bar{J}_{l \rightarrow u}=\frac{g_{u}}{g_{l}} \frac{c^{2} A_{u \rightarrow l}}{2 h \nu^{3}} \int \mathrm{d} \nu J_{\nu} \phi(\nu),
$$

where $\bar{J}_{l \rightarrow u}$ is line profile weighted mean intensity and $\phi(\nu)$ is the Voigt profile.

Equation 2.8 is evaluated for all $0 \leq \ell \leq n-1$ and $1 \leq n \leq 6$, resulting in a linear system of 21 equations in total for the number density of each $(n, \ell)$ state, $n_{n \ell}$. The quantities $n_{e}, n_{p}$, and $T$ are treated as given parameters in the equations. The linear system is solving using Gauss-Jordan elimination (Press et al. 2007).

The rates of important processes related to the $2 p$ state population are listed in Table 2.2. The rates related to higher excited states are not listed in the table because they cannot have a large net effect on $2 p$ in steady state, unless the higher state itself has a large source or sink, which is not the case. The stellar Ly $\alpha$ mean intensity (see Section 2.4) is applied for the estimate. The table shows that all other rates except the $\ell$-mixing rates between $2 s$ and $2 p$ are negligible compared to the radiative rates between $2 p$ and $1 s$. The proton collisional $\ell$-mixing rate is much larger than the electron rate. The $\ell$-mixing rates between $2 s$ and $2 p$ nearly cancel each other and $2 \ell$ states are in collisional equilibrium due to the large $\ell$-mixing rates at the densities of interest. Thus, $A_{2 p \rightarrow 1 s} n_{2 p}$ and $B_{1 s \rightarrow 2 p} \bar{J}_{L y \alpha} n_{1 s}$ completely dominate the $2 p$ generation and destruction rate, and the $\mathrm{n}=2$ state number densities are simply set by $\bar{J}_{L y \alpha}$, so 
Table 2.1: Photoionization rates and photoelectric heating rates

\begin{tabular}{ccc}
\hline \hline Species & $\Gamma_{\mathrm{pi}}\left(\mathrm{s}^{-1}\right)$ & $Q_{\mathrm{pi}}\left(\mathrm{erg} \mathrm{s}^{-1}\right)$ \\
\hline $\mathrm{H}(1 s)$ & $3.73 \times 10^{-4}$ & $2.72 \times 10^{-15}$ \\
$\mathrm{H}(2 s)$ & 86.5108 & $5.12 \times 10^{-11}$ \\
$\mathrm{H}(2 p)$ & 72.1913 & $3.97 \times 10^{-11}$ \\
O I & $1.47 \times 10^{-3}$ & $4.26 \times 10^{-14}$ \\
$\mathrm{O}$ II & $6.03 \times 10^{-4}$ & $1.33 \times 10^{-14}$ \\
$\mathrm{C} \mathrm{I}$ & $3.70 \times 10^{-3}$ & $3.04 \times 10^{-14}$ \\
$\mathrm{C} \mathrm{II}$ & $2.61 \times 10^{-4}$ & $8.21 \times 10^{-15}$ \\
$\mathrm{Mg}$ I & $5.21 \times 10^{-4}$ & $2.26 \times 10^{-14}$ \\
$\mathrm{Mg}$ II & $1.32 \times 10^{-4}$ & $1.28 \times 10^{-14}$ \\
$\mathrm{Si} \mathrm{I}$ & $1.56 \times 10^{-2}$ & $6.41 \times 10^{-14}$ \\
$\mathrm{Si} \mathrm{II}$ & $1.08 \times 10^{-4}$ & $8.34 \times 10^{-15}$ \\
$\mathrm{~S} \mathrm{I}$ & $8.72 \times 10^{-3}$ & $6.76 \times 10^{-14}$ \\
$\mathrm{~S} \mathrm{II}$ & $1.36 \times 10^{-4}$ & $8.61 \times 10^{-15}$ \\
$\mathrm{Na}$ I & $3.66 \times 10^{-3}$ & $4.68 \times 10^{-14}$ \\
$\mathrm{~K}$ I & $1.43 \times 10^{-2}$ & $3.08 \times 10^{-14}$ \\
\hline
\end{tabular}

Note. - Atmospheric attenuation, secondary ionization effect and the contribution by Ly $\alpha$ photon are not included.

Table 2.2: Rate which may be important to $n_{2 p}$ population and de-population

\begin{tabular}{cc}
\hline \hline Process & Rates $\left(\mathrm{cm}^{-3} \mathrm{~s}^{-1}\right)$ \\
\hline Radiative excitation $1 s \rightarrow 2 p$ & $1.7 \times 10^{11}\left(n_{1 s} / 10^{10} \mathrm{~cm}^{-3}\right)$ \\
Collisional excitation $1 s \rightarrow 2 p$ & $8.4 \times 10^{4}\left(n_{e} n_{1 s} / 10^{19} \mathrm{~cm}^{-6}\right)$ \\
Radiative recombination to $2 p$ & $6.2 \times 10^{4}\left(n_{e} n_{p} / 10^{18} \mathrm{~cm}^{-6}\right)$ \\
Spontaneous decay $2 p \rightarrow 1 s$ & $6.3 \times 10^{11}\left(n_{2 p} / 10^{3} \mathrm{~cm}^{-3}\right)$ \\
$p$ collisional $\ell$-mixing $2 p \rightarrow 2 s$ & $1.9 \times 10^{8}\left(n_{2 p} n_{p} / 10^{12} \mathrm{~cm}^{-6}\right)$ \\
Photoionization from $2 p$ & $7.2 \times 10^{4}\left(n_{2 p} / 10^{3} \mathrm{~cm}^{-3}\right)$ \\
Collisional de-excitation $2 p \rightarrow 1 s$ & $7.4 \times 10^{3}\left(n_{2 p} n_{e} / 10^{12} \mathrm{~cm}^{-6}\right)$ \\
Collisional ionization from $2 p$ & $7.4 \times 10^{2}\left(n_{2 p} n_{e} / 10^{12} \mathrm{~cm}^{-6}\right)$ \\
\hline
\end{tabular}

Note. - In the table, $\bar{J}_{L y \alpha}=2 \times 10^{-9} \mathrm{erg} \mathrm{cm}^{-2} \mathrm{~s}^{-1} \mathrm{~Hz}^{-1}$ and $T=8000 \mathrm{~K}$ are applied. The reference numbers are $n_{1 s}=10^{10} \mathrm{~cm}^{-3}, n_{e}=n_{p}=10^{9} \mathrm{~cm}^{-3}$ and $n_{2 p}=10^{3} \mathrm{~cm}^{-3}$. 
we have,

$$
n_{2 p} \approx 3 n_{2 s} \approx n_{1 s} \frac{g_{u}}{g_{l}} \frac{c^{2} \bar{J}_{L y \alpha}}{2 h \nu^{3}} .
$$

In Section 2.5, it will be shown that this is a good approximation for the whole simulation region. To obtain the intensity of $\operatorname{Ly} \alpha$, a resonant scattering study of Ly $\alpha$ photon will be discussed in Section 2.4.

\subsection{The Atmosphere Model}

\subsubsection{Basic Structure}

Following Christie et al. (2013), a spherically-symmetric, hydrostatic atmosphere model is constructed for the region composed of ionized and atomic gas sitting above the molecular atmosphere. The transit radius measured in broadband optical wave-

lengths is $R_{p}$, and the base of the atomic layer is at radius $R_{b}>R_{p}$. The thickness of the molecular layer below the atomic layer is then $R_{b}-R_{p}$. Assuming an isothermal molecular layer with equilibrium temperature $T=T_{\text {eq }}=1140 \mathrm{~K}$ (Wyttenbach et al. 2015), the thickness is

$$
R_{b}-R_{p} \approx \frac{k_{\mathrm{B}} T_{\mathrm{eq}} R_{p}^{2}}{\mu m_{\mathrm{H}} G M_{p}} \ln \left(\frac{P_{p}}{P_{b}}\right)
$$

where $\mu \approx 2.3$ is the mean molecular weight, $P_{p}=1$ bar is the pressure of the optical photosphere suggested by Sharp \& Burrows (2007). It is assumed that the pressure at the base of the atomic layer is $P_{b}=10 \mu$ bar. It will be shown that the temperature in the atomic layer becomes small enough for molecular hydrogen to dominate there, for the assumptions used here. 


\subsubsection{Differential Equations}

Given the temperature and number density of each species at one level in the atmosphere, the equation of hydrostatic balance and equations for the column of each species must be integrated inward to find pressure and columns at the next step inward. The hydrostatic balance equation is

$$
\frac{\mathrm{d} P}{\mathrm{~d} r}=-\frac{\rho G M_{p}}{r^{2}}
$$

and the columns are integrated as

$$
\frac{\mathrm{d} N_{i}}{\mathrm{~d} r}=-n_{i}
$$

The subscript $i$ stands for each species, including $\mathrm{H}(1 \mathrm{~s})$ and the individual neutral and first ionized metal element considered. The ideal gas law for the gas pressure is

$$
P=\left(n_{e}+\left(n_{p}+n_{\mathrm{H}}\right)\left(1+f_{z}+f_{\mathrm{He}}\right)\right) k_{\mathrm{B}} T
$$

where $f_{z}=\sum f_{m}$ is the sum of metal species relative number density abundance to hydrogen, and $f_{\mathrm{He}}$ is the fraction of He by number assuming solar abundance (Asplund et al. 2009). Ionization of He is ignored in this paper. The gas density is written

$$
\rho=\left(n_{p}+n_{\mathrm{H}}\right)\left(1+4 f_{\mathrm{He}}+\sum m_{m} f_{m}\right) m_{p}
$$

where $m_{m}$ is the metal atomic mass in atomic units.

The pressure and the column density are integrated inward, with a starting value $P_{\text {top }}=5 \times 10^{-5} \mu$ bar on the outside, where the atmosphere above becomes optically thin to Ly $\alpha$. The starting value of each $N_{i}=0$. The solution is integrated inward 
from a starting radius $R_{\text {top }}$ and pressure $P_{\text {top }}$, until the base radius $R_{b}$ is reached. The boundary condition imposed there is that $P=P_{b}$. This boundary condition is satisfied in practice by varying the starting radius $R_{\text {top }}$ until $P\left(R_{b}\right)=P_{b}$, the desired value, using Brent's method (Press et al. 2007).

The hydrostatic model will be inaccurate near the outer boundary, as a number of physical effects have been neglected, such as: outflowing gas from the planet, interaction with the stellar wind, strong magnetic forces, radiation pressure, and stellar tidal forces. The region where these effects may be appreciable will be estimated in Section 2.7.1. However, in the region where this model shows the dominant absorption by $\mathrm{H}(2 \ell)$, the density is so high that these effects are negligible. Hence the hydrostatic model is sufficient for the purposes of this study.

\subsubsection{Ionization State and Temperature}

At each level of the atmosphere, the pressure $P$ and columns $N_{i}$ are given by the boundary conditions or the integration of Equations 2.16 and 2.17. The temperature and particle densities must then be updated to continue the integration. Since the gas is not in local thermodynamic equilibrium (LTE), these quantities must be determined by solving rate equilibrium equations for ionization/recombination, heating/cooling, a charge balance equation, and the equation of state. The equations used are as following. Terms related to metal species will be discussed in more detail in Section 2.3.4.

The charge balance equation is

$$
n_{p}+\sum n_{\mathrm{M}_{\mathrm{II}}}+2 \sum n_{\mathrm{M}_{\mathrm{III}}}=n_{e}
$$

where $n_{\mathrm{M}_{\mathrm{II}}}$ and $n_{\mathrm{M}_{\mathrm{III}}}$ are the number density of first ionized and second ionized metal 
species respectively. Higher ionization states are ignored as their abundance would be negligible for the given ionizing flux and particle densities.

The hydrogen ionization and recombination balance equation is

$$
\begin{aligned}
\left(\alpha_{B} n_{e}+k_{\mathrm{ion}}^{(\mathrm{O})} n_{\mathrm{OI}}\right) n_{p}= & n_{1 s}\left(\Gamma_{1 s}+C_{1 s \rightarrow \infty}^{(e)} n_{e}+k_{\mathrm{rec}}^{(\mathrm{O})} n_{\mathrm{O} \text { II }}\right) \\
& +C_{2 \rightarrow \infty}^{(e)} n_{e} n_{2}+\Gamma_{2 p} n_{2 p}+\Gamma_{2 s} n_{2 s}+\sum_{m} \Gamma_{m}^{2 n d} n_{m}
\end{aligned}
$$

where $\alpha_{B}$ is the case $\mathrm{B}$ recombination rate, $k_{\text {ion }}^{(\mathrm{O})}$ and $k_{\text {rec }}^{(\mathrm{O})}$ are the rates of oxygen ionize and recombine through charge exchange with hydrogen respectively. The $\mathrm{n}=2$ state has separate contributions from $\mathrm{H}(2 \mathrm{~s})$ and $\mathrm{H}(2 \mathrm{p})$ as

$$
C_{2 \rightarrow k}^{(e)} n_{e} n_{2}=C_{2 s \rightarrow k}^{(e)} n_{e} n_{2 s}+C_{2 p \rightarrow k}^{(e)} n_{e} n_{2 p}
$$

The last term in Equation 2.21 represents ionization from the $\mathrm{H}(1 \mathrm{~s})$ state due to photoelectrons created by metal ionization. Hence high energy photoelectrons created through ionization of metal species can have the same secondary ionization effect as Equation 2.11. The secondary ionization rate due to metal species is then

$$
\Gamma_{m}^{2 \mathrm{nd}}=\int_{\nu_{\mathrm{th}}}^{\infty} \sigma_{m, \mathrm{pi}}(\nu) k_{e} \frac{F_{\nu}}{h \nu} e^{-\tau_{\mathrm{att}, \nu} \mathrm{d} \nu}
$$

where $\sigma_{m, \mathrm{pi}}(\nu)$ is the metal photoionization cross section. When evaluating the excited state $\mathrm{H}$ abundance in Equation 2.21, the approximation in Equation 2.14 is applied. 
The heating and cooling balance equation is

$$
\begin{aligned}
& {\left[\left(13.6 \mathrm{eV} C_{1 s \rightarrow \infty}^{(e)}+10.2 \mathrm{eVC}_{1 s \rightarrow 2}^{(e)}\right) n_{1 s}+3.4 \mathrm{eV} C_{2 \rightarrow \infty}^{(e)} n_{2}+\sum_{m} \Lambda_{m} n_{m}\right.} \\
& \left.\quad+\Lambda_{f f} n_{p}+\left\langle E_{r r}\right\rangle \alpha_{B} n_{p}+k_{\mathrm{B}} T \sum_{m} \alpha_{m} n_{m}^{\text {ion }}\right] n_{e} \\
& =n_{1 s} Q_{1 s}\left(N_{1 s}\right)+Q_{2 p} n_{2 p}+Q_{2 s} n_{2 s}+10.2 \mathrm{eV} n_{e} n_{2} C_{2 \rightarrow 1}^{(e)}+\sum_{m} Q_{m} n_{m}
\end{aligned}
$$

where $\Lambda$ stands for cooling function. Osterbrock \& Ferland (2006) give the free free cooling rate

$$
\Lambda_{f f}=1.85 \times 10^{-27} \mathrm{~T}^{1 / 2}\left(\mathrm{erg} \mathrm{cm}^{-3} \mathrm{~s}^{-1}\right)
$$

where $T$ is in units of Kelvin. The mean kinetic energy of the recombining electrons is (Draine 2011)

$$
\left\langle E_{r r}\right\rangle=\left[0.684-0.0416 \ln \left(T_{4}\right)\right] k_{\mathrm{B}} T
$$

where $T_{4}=T / 10^{4} \mathrm{~K}$. In Equation 2.24, the symbol $Q$ represents the photoelectric heating rate, per photoionization, corrected for the secondary ionization effect. Dalgarno et al. (1999) find that secondary electrons give rise to roughly the same number of ionizations as $1 \mathrm{~s} \rightarrow 2 \mathrm{p}$ excitations, so the heat deposited into the atmosphere by one photoelectron with energy $E$ is taken to be

$$
\eta(E)=E-(13.6 \mathrm{eV}+10.2 \mathrm{eV}) k_{e}
$$

where the second term represents the energy lost by the photoelectron to ionizations and Ly $\alpha$ excitations. Thus, the net photoelectric heating rate is

$$
Q=\int_{\nu_{\mathrm{th}}}^{\infty} \eta \sigma_{p i}(\nu) \frac{F_{\nu}}{h \nu} e^{-\tau_{\mathrm{att}, \nu} \mathrm{d} \nu}
$$


Given $P$ and the columns $N_{i}$, Equations 2.18, 2.20, 2.21, and 2.24 give four algebraic equations to solve for $T, n_{e}, n_{p}$, and $n_{1 s}$ at this level in the atmosphere. A globally convergent Newton's method (Press et al. 2007) is applied to solve the set of equations.

\subsubsection{Radiative Cooling Due to Metal Species}

Although $\mathrm{H}$ and $\mathrm{He}$ are by far the most abundant elements, their electron-impact line cooling rates are heavily suppressed at temperatures $T \lesssim 10^{4} \mathrm{~K}$ due to the high excitation energies. Metal line cooling due to electron impact followed by radiative de-excitation is an important coolant, especially near the base of the atmosphere at $T \lesssim 8000 \mathrm{~K}$. The ionization/recombination rate equilibrium equation is included to determine the relative abundance of each ionization state. Transitions yielding large cooling rates are chosen from abundant elements, and by striking a balance between low excitation energies, $\Delta E$, and large radiative decay rate $A_{u l}$. Solar abundance is assumed (Asplund et al. 2009). The elements considered are O, C, Mg, Si, S, $\mathrm{Na}$, and K. Although Mg was not a priori expected to be abundant in the upper atmosphere due to condensation (Visscher et al. 2010; Koskinen et al. 2013b), $\mathrm{Mg}_{\mathrm{I}}$ is in fact detected in HD 209458b (Vidal-Madjar et al. 2013) and marginally detected in HD 189733b (Cauley et al. 2016).

The abundance of each ionization state is set by solving for rate equilibrium between ionization and recombination. Only neutral, first, and second-ionized atoms are included.

As a special case, rather than photoionization, collisional, and radiative recombination, the ionization state of oxygen is determined by charge exchange with hydrogen. Considering that $n_{\mathrm{H}} \gtrsim 10^{5} \mathrm{~cm}^{-3}$ everywhere in the model, the charge exchange 
rates in the high-density limit in Draine (2011) are applied. The energy differences between three fine-structure levels of neutral oxygen are ignored because they are much smaller than $k_{\mathrm{B}} T$.

The rate equilibrium equations are

$$
\begin{aligned}
\left(\Gamma_{\mathrm{M}_{\mathrm{I}}}+C_{\mathrm{M}_{\mathrm{I}}, \infty}^{(e)} n_{e}+k_{\mathrm{ion}}^{(\mathrm{O})} n_{p}\right) n_{\mathrm{M}_{\mathrm{I}}} & =\left(\alpha_{\mathrm{M}_{\mathrm{II}}} n_{e}+k_{\mathrm{rec}}^{(\mathrm{O})} n_{1 \mathrm{~s}}\right) n_{\mathrm{M}_{\mathrm{II}}} \\
\Gamma_{\mathrm{M}_{\mathrm{II}}} n_{\mathrm{M}_{\mathrm{II}}} & =\alpha_{\mathrm{M}_{\mathrm{III}}} n_{e} n_{\mathrm{M}_{\mathrm{III}}} \\
n_{\mathrm{M}_{\mathrm{I}}}+n_{\mathrm{M}_{\mathrm{II}}}+n_{\mathrm{M}_{\mathrm{III}}} & =\left(n_{\mathrm{H}}+n_{p}\right) f_{m},
\end{aligned}
$$

where $C_{\mathrm{M}_{\mathrm{I}}, \infty}^{(e)}$ is the electron collisional ionization rate, which is only considered for $\mathrm{Na}$ and $\mathrm{K}$ atoms. The collisional ionization of other metal species are ignored because of the much higher ionization potential. The secondary ionization states of $\mathrm{Na}$ and $\mathrm{K}$ are ignored. The photoionization rates of all species from Verner et al. $(1995,1996)$. The Na and K collisional ionization rates are given by Lennon et al. (1988).

The rates of Pequignot et al. (1991) are used to describe the recombination of C and O, of Shull \& van Steenberg (1982) for that of Mg, Si, and S, of Verner \& Ferland (1996) and Landini \& Fossi (1991) for that of Na and K respectively.

Because the ionization potentials of Mg I, Si I, Na I, and K I are smaller than the Ly $\alpha$ energy, both continuum and Ly $\alpha$ photons contribute to their photoionization and photoelectric heating rates. The metal species photoionization rate is

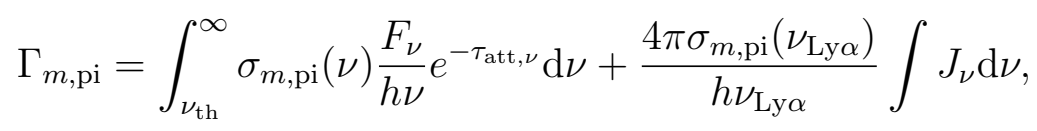

and the metal species photoelectric heating rate

$$
Q_{m, \mathrm{pi}}=\int_{\nu_{\mathrm{th}}}^{\infty} \eta \sigma_{m, \mathrm{pi}}(\nu) \frac{F_{\nu}}{h \nu} e^{-\tau_{\mathrm{att}, \nu}} \mathrm{d} \nu+4 \pi \sigma_{m, \mathrm{pi}}\left(\nu_{\mathrm{Ly} \alpha}\right) \frac{\nu_{\mathrm{Ly} \alpha}-\nu_{\mathrm{th}}}{\nu_{\mathrm{Ly} \alpha}} \int J_{\nu} \mathrm{d} \nu
$$


where $\sigma_{m, \mathrm{pi}}\left(\nu_{\mathrm{Ly} \alpha}\right)$ is only nonzero for Mg I, Si I, Na I, and K I. The mean intensity $J_{\nu}$ in these formulas denotes the intensity in the vicinity of the Ly $\alpha$ line, found as a result of the resonant scattering calculation in Section 2.4.

The metal line cooling rates require a model for the excited state densities. For a two-level system, rate equilibrium between upward and downward rates gives

$$
n_{l}\left(n_{e} C_{l u}^{(e)}+B_{l u} \bar{J}_{l u}\right)=n_{u}\left(A_{u l}+n_{e} C_{u l}^{(e)}\right) .
$$

Stimulated emission is ignored due to dilution of the stellar flux. Collisional excitation is a sink of thermal translation energy while collisional de-excitation is a source. Thus the cooling rate of this two levels system is

$$
\Delta E n_{e}\left(C_{l u}^{(e)} n_{l}-C_{u l}^{(e)} n_{u}\right)=n_{e} n_{l} \Delta E C_{l u}^{(e)}\left(\frac{A_{u l}-B_{l u} \bar{J}_{l u}\left(n_{l} / n_{u}\right)_{\mathrm{eq}}}{A_{u l}+n_{e} C_{u l}^{(e)}}\right) \equiv \Lambda n_{e} n_{l}
$$

where $\left(n_{l} / n_{u}\right)_{\mathrm{eq}}=g_{l} / g_{u} \exp \left(\Delta E /\left(k_{\mathrm{B}} T\right)\right)$, and the last equality defines $\Lambda(T)$, the cooling function. Permitted transitions in the optical and near UV bands are always associated with strong absorption features in the stellar spectrum, which lead to a very small radiative excitation rate $B_{l u} \bar{J}_{l u}\left(n_{l} / n_{u}\right)_{\text {eq }}$ for the transitions used. In the case of forbidden transitions, to compensate for the small $A_{u l}$, only small $\Delta E$ transitions give rise to significant cooling. At the long wavelength end, the dilution of radiation flux due to the solid angle of the star overcomes the effect of higher brightness temperature of the star. As a result, radiative excitation is negligible in Equation 2.33 for both permitted and forbidden transitions.

For forbidden transitions, the electron number density $n_{e}$ is much larger than the critical density $n_{\text {crit }}$ above which collisional de-excitation dominates radiative deexcitation. In this limit, the level population is given by the Boltzmann distribution, 
and the cooling rate becomes

$$
\Lambda n_{e} n_{l}=\Delta E n_{l} A_{u l}\left(\frac{n_{u}}{n_{l}}\right)_{\mathrm{eq}}
$$

which is independent of $n_{e}$ and collisional rates.

The emitted metal line photons are assumed to escape freely from the atmosphere. In reality, the atmosphere may be optically thick to permitted emissions near the base of the atomic layer.

The major cooling processes are listed in Table 2.3, while Table 2.4 in the appendix contains addition transitions from $\mathrm{O}, \mathrm{C}, \mathrm{S}$, and $\mathrm{Si}$ lines which are included in the model but only have a minor effect on the temperature. The lower state of some transitions may not be the ground state. In this case, the lower state is assumed to reach collisional equilibrium with ground state in the cooling rate calculation. Table 2.4 contains the transitions from O, C, S, and Si lines, which are included in the model as cooling processes but only have a minor effect on the temperature.

Mg I $5184 \AA$ is another line that may contribute to cooling. The lower state of this transition is not the ground state, and the upper state is associated with the ground

state of the strong resonance line Mg I $2852 \AA$. A Mg level population calculation and Mg I $2852 \AA$ radiation transfer study are required to accurately model the cooling effect, which is beyond the scope of this work. $\mathrm{Mg} \mathrm{I} 5184 \AA$ is not included in this work as a result.

\subsubsection{Molecular Hydrogen}

Near the base of the atomic layer, where the temperature drops below $T \sim 2000-$ $3000 \mathrm{~K}$, it is expected that the density of molecules will increase rapidly and come to dominate over the atomic species. This is the base of the atomic layer and the 
top of the molecular layer. The atomic-to-molecular transition is not self-consistently modeled in this work, as the rate equations to determine molecular densities (e.g. Yelle 2004; García Muñoz 2007), and the strong effect of molecular coolants from e.g. $\mathrm{H}_{2} \mathrm{O}$ rotation-vibration bands, are not taken into account.

Although the details of molecule formation are beyond the scope of this paper, a rough estimate of the $\mathrm{H}_{2}$ number density is made to verify that the temperature does indeed become low enough to form molecules as the base is approached. Lenzuni et al. (1991) noted that for a wide range of radiation intensity, that dissociation of $\mathrm{H}_{2}$ is due to collisional processes, rather than photo-dissociation. Yelle (2004) found the same result for a model for the thermosphere of HD 209458b. In this limit, an estimate of the $\mathrm{H}_{2}$ density can be found using the Saha equation. Applying the $\mathrm{H}_{2}$ partition function from Borysow et al. (1989), the $\mathrm{H}_{2}$ number density, $n_{\mathrm{H}_{2}}$ can be computed from the atomic hydrogen density, $n_{\mathrm{H}}$, and the temperature $T$.

The buildup of a significant column of $\mathrm{H}_{2}$ will shield the lower atmosphere from stellar UV, and allow the formation of other molecules, e.g. $\mathrm{CO}$ and $\mathrm{H}_{2} \mathrm{O}$ which may be important coolants. Another effect more relevant to this work is that $\mathrm{H}_{2}$ may act as a strong absorber of Ly $\alpha$ photons, effectively setting a lower boundary to the region of the atmosphere that may have a large Ly $\alpha$ intensity. Black \& van Dishoeck (1987) discussed numerous "accidental resonances" between Ly $\alpha$ and electronic transitions in $\mathrm{H}_{2}$. The strongest of these can have oscillator strength $f \sim 10^{-2}$, implying that a column $N_{\mathrm{H}_{2}} \simeq 10^{14} \mathrm{~cm}^{-2}$ is required to give unit optical depth for these transitions. For a scale height $H \simeq 10^{8} \mathrm{~cm}$, this gives a critical number density $n_{\mathrm{H}_{2}} \simeq 10^{6} \mathrm{~cm}^{-3}$ for true absorption optical depth unity over a scale height. In practice, Ly $\alpha$ photons near the optically-thick base of the atomic layer would require a large number of scatterings to escape the atmosphere, implying a total distance traversed even larger 
than a scale height. This will effectively set an absorbing lower boundary for the atomic layer in the radiation transfer described in Section 2.4.

\subsection{Ly $\alpha$ Radiation Transfer}

Ly $\alpha$ photons can excite hydrogen from the 1 s to the $2 \mathrm{p}$ state, providing a population of absorbers that may be detected in Balmer line transmission spectra. Ly $\alpha$ may also play a role in the heating/cooling and ionization/recombination balance, so a detailed Ly $\alpha$ radiation transfer calculation is crucial.

Two sources of Ly $\alpha$ are included in the model, the stellar Ly $\alpha$ incident through the top of the planet's atmosphere, and also Ly $\alpha$ generated within the planet's atmosphere by electron impact excitation or a recombination cascade. The results of particle densities and temperature versus radius from the hydrostatic model in Section 2.3 are used to specify the Ly $\alpha$ source function, as well as the mean free paths to scattering and true absorption. In the transfer calculation it is convenient to use Ly $\alpha$ line center optical depth, $\tau$, as the vertical coordinate. A plot of pressure $\mathrm{P}$ and radius $\mathrm{r}$ versus $\tau$ will be shown in Figure 2.3. A major simplifying assumption is to use plane-parallel geometry, so that mean intensity $J_{\nu}(r)$ is tabulated as a function of altitude. The radius in the hydrostatic model varies by a factor of 2 from base to top, and by a factor of $\simeq 20 \%$ near the base of the layer where the $\mathrm{H} \alpha$ line is formed. The plane-parallel assumption simplifies the calculation, and is consistent with uniform irradiation assumed in the hydrostatic model.

At the outer boundary $(\tau=0)$, the unpolarized stellar Ly $\alpha$ intensity enters the slab vertically. The line is parameterized by a double Gaussian line profile with width (in velocity units) $\sigma=49 \mathrm{~km} \mathrm{~s}^{-1}$ and centers at $\mu= \pm 74 \mathrm{~km} \mathrm{~s}^{-1}$ (Gladstone 1988; Curdt et al. 2010; Tian et al. 2009). The integrated line flux at the top of 
the HD 189733b's atmosphere is $F_{0}=2.0 \times 10^{4} \mathrm{erg} \mathrm{cm}^{-2} \mathrm{~s}^{-1}$ (Linsky et al. 2013). Outgoing photons can escape from the top boundary freely.

An absorption bottom boundary is applied at the base of the slab, which represents the base of the atomic layer. Physically, this boundary condition is imposed to represent the short mean free path to true absorption of $\mathrm{Ly} \alpha$ on $\mathrm{H}_{2}$ (Shull 1978; Black \& van Dishoeck 1987) and $\mathrm{H}_{2} \mathrm{O}$ (Miguel et al. 2015). Since $n_{\mathrm{H}_{2}}$ increases inward much faster than $n_{\mathrm{H}}$, the mean free path to resonant scattering will become longer than that to true absorption in the molecular layer, greatly decreasing the Ly $\alpha$ intensity compared to the atomic layer. As will be shown, even in the atomic layer, the intensity falls rapidly toward the base.

Ly $\alpha$ generated inside the atmosphere by collisional excitation or a recombination cascade is assumed to be unpolarized and have an initial frequency distribution given by a Gaussian distribution with the Doppler width set by the local temperature. In practice, this initial distribution is much narrower than the resultant mean intensity, so it is the same effect as initializing photons at line center. The source function inside the atmosphere is

$$
S_{\nu}=\frac{10.2 \mathrm{eV}}{4 \pi}\left(\alpha_{B} n_{p} n_{e}+C_{1 s \rightarrow 2}^{(e)} n_{1 s} n_{e}+\Gamma_{1 s}^{2 \mathrm{nd}} n_{1 s}+\sum_{m} \Gamma_{m}^{2 \mathrm{nd}} n_{m}\right) \phi_{\nu},
$$

where $\phi_{\nu}$ is the Doppler profile evaluated at the local temperature. The first term in Equation 2.35 represents recombinations, each of which is assumed to produce one Ly $\alpha$ photon. The following three terms represent collisional excitation by thermal electrons, photoelectrons from ionizing $\mathrm{H}(1 \mathrm{~s})$, and photoelectrons generated from ionizing metals. Recombination and collisional excitation to $\mathrm{H}(2 \mathrm{~s})$ are also included because $\mathrm{H}(2 \mathrm{~s})$ and $\mathrm{H}(2 \mathrm{p})$ are well coupled by the $\ell$-mixing.

The line center optical depth to scattering reaches values as large as $\tau \simeq 10^{8}$ 
near the base of the model, and photons will scatter $\sim \tau$ times before exiting the atmosphere (Harrington 1973) if no other process intervenes. Ly $\alpha$ photons can leave this scattering cycle by two categories of processes. First, the radiative excitation may not be followed by radiative de-excitation of a Ly $\alpha$ photon some fraction $\epsilon$ of the time. The dominant processes are: photoionization from the $\mathrm{n}=2$ state by stellar Balmer continuum emission; collisional de-excitation; and two photon decay from $2 \mathrm{~s} \rightarrow 1 \mathrm{~s}$. The former two processes also contribute to ionizing and heating rates. The second kind of process is "true absorption", in which a Ly $\alpha$ photon is absorbed by some other species besides $\mathrm{H}(1 \mathrm{~s})$, for example by photoionizing an atom with low ionization potential less than $10.2 \mathrm{eV}$.

Ly $\alpha$ photons can also leave this scattering cycle by the $\ell$-mixing or radiative excitation processes from the $2 \mathrm{p}$ state. Because it will be followed by the reverse process immediately, these processes are equivalent to Ly $\alpha$ photon redistribution that put the line wing photons frequently back to the line center and stop the photons from escaping. These processes are not included in this model because a careful consideration required the $\mathrm{H} \alpha$ mean intensity in the atmosphere, which is not available right now.

The plane-parallel transfer equation including resonant scattering, true absorption, the source function for photon creation, and excitation followed by de-excitation a fraction $1-\epsilon$ of the time is

$$
\begin{aligned}
\mu \frac{\mathrm{d} I_{\nu}(z, \boldsymbol{n})}{\mathrm{d} z}= & -\left(\alpha_{\mathrm{sc}}+\alpha_{\mathrm{abs}}\right) I_{\nu}(z, \boldsymbol{n})+S_{\nu}(z) \\
& +(1-\epsilon) 4 \pi \frac{\alpha_{\mathrm{sc}}}{\phi_{\nu}} \int R\left(\nu, \boldsymbol{n} ; \nu^{\prime}, \boldsymbol{n}^{\prime}\right) I_{\nu^{\prime}}\left(z, \boldsymbol{n}^{\prime}\right) \mathrm{d} \Omega^{\prime} \mathrm{d} \nu^{\prime}
\end{aligned}
$$

where $\mu=\cos \theta, \phi_{\nu}$ is the Voigt line profile, $\alpha_{\mathrm{sc}}$ is resonant scattering coefficient, $\alpha_{\mathrm{abs}}$ is true absorption coefficient, and $R$ is the Hummer (1962) case II-B redistribution 
function, with dipole angular dependence. The redistribution function $R$ gives the probability that the photon is scattered from incident frequency $\nu^{\prime}$ and direction $\boldsymbol{n}^{\prime}$ to frequency $\nu$ and direction $\boldsymbol{n}$. Case II-B redistribution assumes that, in the rest frame of the atom, the line profile for absorption of the photon (excitation) is a Lorentzian profile, and that in the rest frame the outgoing photon has the same energy as the ingoing photon. Hummer (1962) presents formulae for the resulting redistribution function thermally averaged over a Boltzmann distribution of atom velocities. Case II-B with dipole angular dependence results for resonant scattering when the initial and final states are the $\mathrm{H}(1 \mathrm{~s})$ state, and the finite lifetime of the intermediate $\mathrm{H}(2 \mathrm{p})$ state is included. Fine structure splitting of the excited state is ignored. This is a good approximation in the present application where the mean intensity is much broader than the fine structure separation of the $J=1 / 2$ and $3 / 2$ states.

The transfer equation is solved numerically with the Monte Carlo method (e.g. Whitney 2011). First, unpolarized photon packets are initialized at a point randomly generated from the source function (see Equation 2.35) and with a randomly chosen direction. Second, the optical depth $\tau$ that the photon will travel through before it is scattered or absorbed is randomly generated with a probability density $e^{-\tau}$. The spatial location of the scattering or "true absorption" at optical depth $\tau$ from the point of emission is then determined with the knowledge of the densities and temperature, and cross sections of $n_{\mathrm{H}}, n_{\mathrm{MgI}}, n_{\mathrm{SiI}}, n_{\mathrm{NaI}}, n_{\mathrm{KI}}$. The Lucy method (Lucy 1999) is used to tabulate intensity and flux from the motion of the photon packets. This was crucial in optically thick regions, and worked much better than accumulating photon statistics only when they pass through the face of a cell. Based on the radiative excitation and absorption optical depth, the rejection method is used to determine whether the photon is absorbed. Whether the $\mathrm{H}(2 \mathrm{p})$ emits another Ly $\alpha$ photon is determined by 
comparing a random number with $1-\epsilon$. Third, at each scattering, the outgoing photon direction is chosen including polarization accumulated during prior scatterings. The Stokes matrix for Rayleigh scattering is used. However, rather than using the Stokes matrix in scattering-plane coordinates, as discussed in Chandrasekhar (1960), a scattering matrix was derived in terms of the incoming and outgoing photon direction without reference to the scattering plane, which was found to be more convenient for numerical calculations. Then, the velocity of scatterers along the direction of the incident photon is randomly generated by the method described in Zheng \& Miralda-Escudé (2002), with small modifications to improve the efficiency. Recoil is included in computing the new frequency of the photon after scattering. The process of propagation and scattering is repeated until the photon escapes the modeled system or leaves the scattering cycle.

By knowing the $n_{1 s}$ from the hydrostatic atmosphere model and $\bar{J}_{L y \alpha}$ from the Ly $\alpha$ radiation transfer calculation, the $n_{2 \ell}$ population is given by Equation 2.14. Note that the ionization, heating, and cooling rates in the atmosphere depend on the $n_{2 \ell}$ and Ly $\alpha$ mean intensity. Therefore, the hydrostatic model and radiation transfer calculation are performed iteratively, until the $n_{2 \ell}$ converged, which takes typically $\sim 6$ iterations.

\subsection{Fiducial Atmosphere Model}

In this section, results are presented for the fiducial model with solar abundance for all species and extreme ultraviolet (EUV) and X-ray flux set by the day-side value. The Ly $\alpha$ mean intensity spectrum in the atmosphere is shown in Figure 2.2. Temperature versus height for the fiducial model is shown in Figure 2.3. The heating

and cooling rates per unit volume are given in Figure 2.4, and the number densities of 


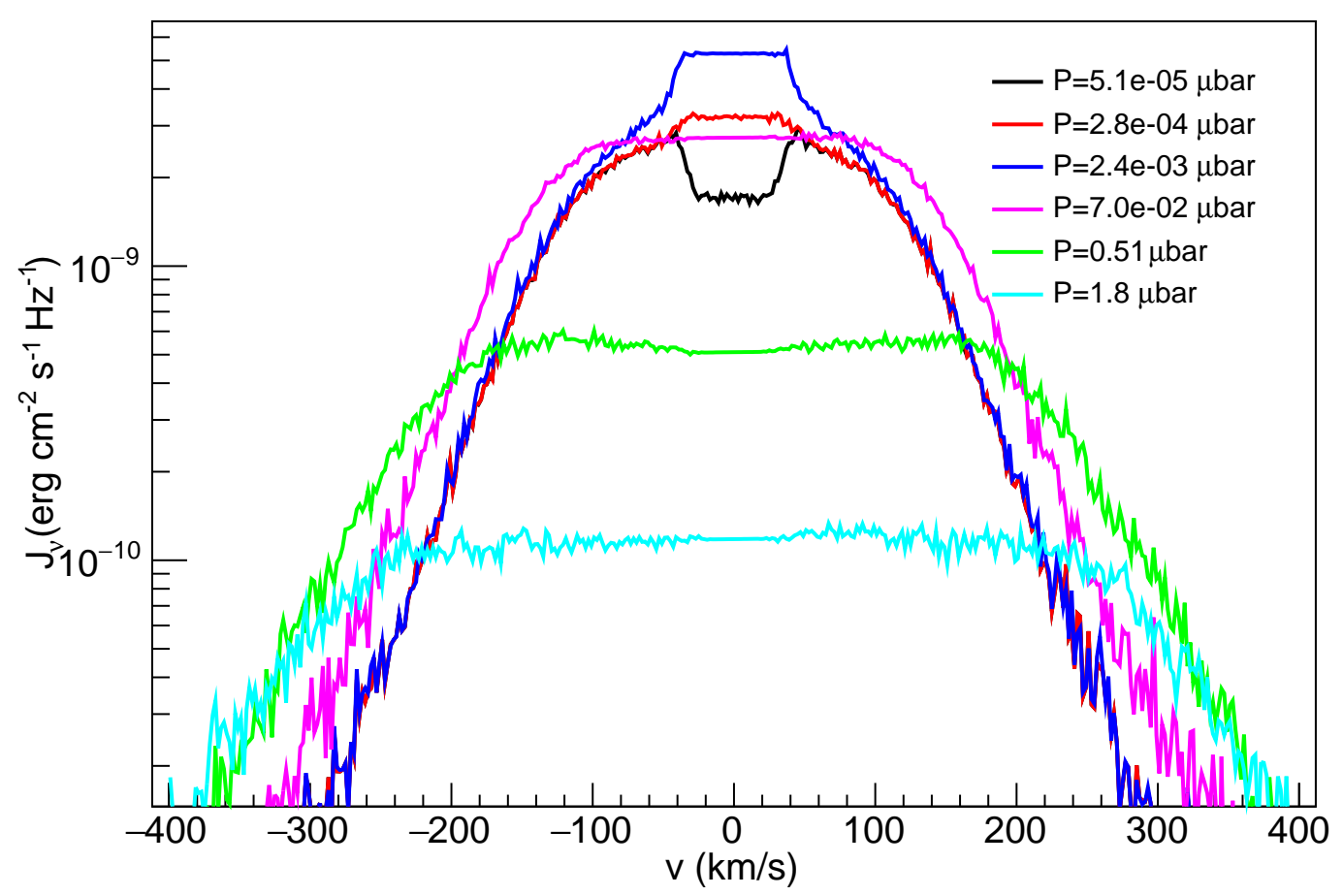

Fig. 2.2.- Ly $\alpha$ mean intensity spectrum $\left(J_{\nu}\right)$ at six different depths in the atmosphere. At line center the spectrum is flat, much wider than the thermal line width, and becomes broader with depth. The intensity decreases rapidly on the line wing. The fluctuations in the spectra are due to the statistical noise in the Monte Carlo simulation.

each species are given in Figure 2.5. Figure 2.6 shows the Ly $\alpha$ photon sources, sinks, and $\mathrm{H}$ ionization rates per unit volume. Line profile weighted Ly $\alpha$ mean intensity $\bar{J}_{L y \alpha}$ of the fiducial model are given in Figure 2.7.

Figure 2.2 shows the Ly $\alpha$ mean intensity at four different depths in the atmosphere. The line center of the resonant scattering spectrum has a flat part with width $\sim(a \tau)^{1 / 3} \nu_{D}$ (Harrington 1973). The dip at line center near the surface arises because the photons have to diffuse away from line center in order to escape from the slab, due to the extremely short mean free path at line center. The mean intensity near line center has a peak around $\tau \sim 10^{4}$, where the $\mathrm{H}(1 s)$ photoionization, as well as the 


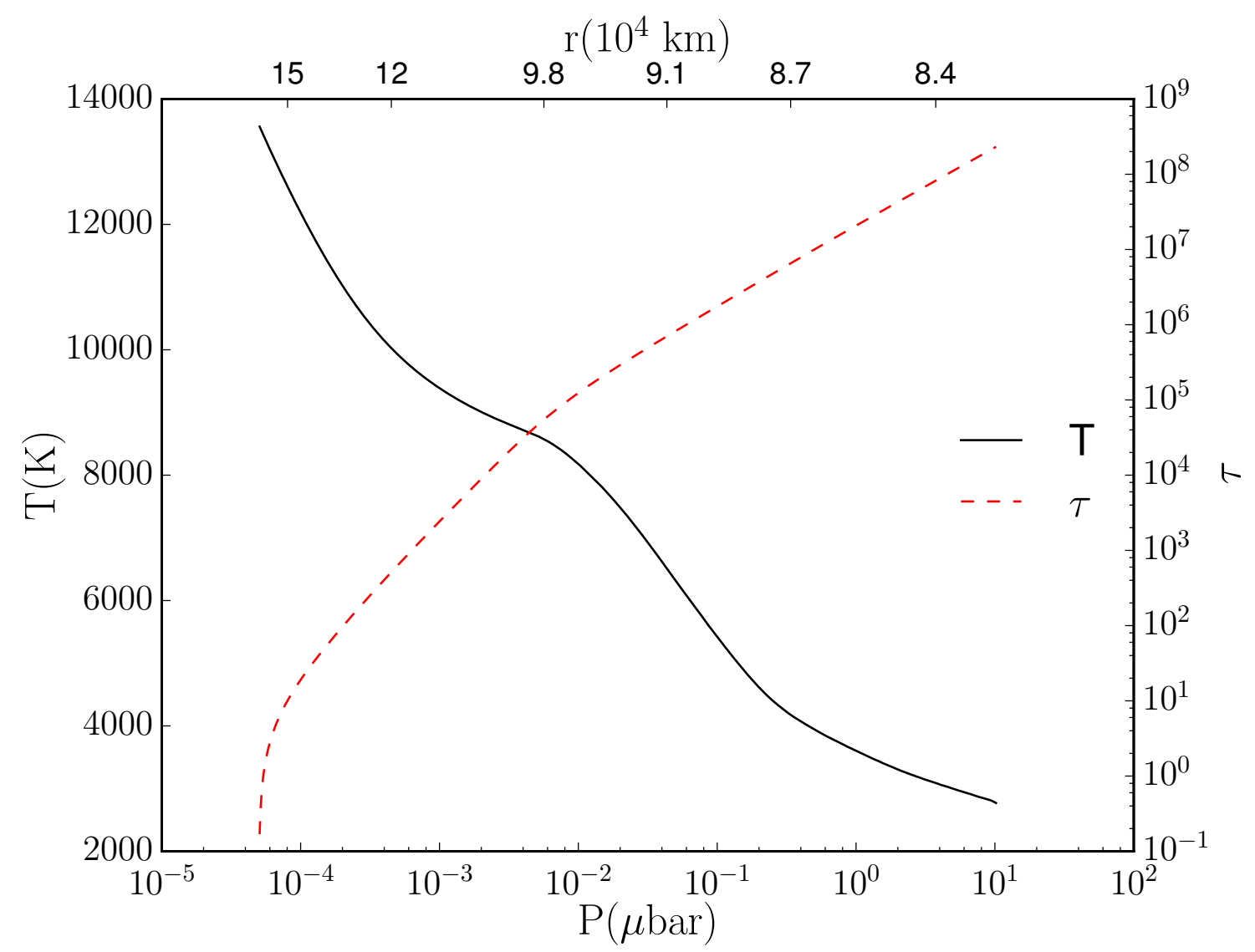

Fig. 2.3. - Temperature $(T)$ and Ly $\alpha$ line center optical depth $(\tau)$ versus pressure $(P$, bottom axis) and radius ( $r$, top axis $)$.

subsequent recombination, which is the major Ly $\alpha$ photon source, is the strongest. The spectrum decreases rapidly on the line wing. The fluctuations in the spectra are due to the statistical noise in the Monte Carlo simulation. Since the number densities of $\mathrm{H}(2 \ell)$ are proportional to the $\bar{J}_{L y \alpha}$, this fluctuation also appears in the $\mathrm{H}(2 \ell)$ number densities curve, and other curves such as heating rates.

At the top of the model, for pressures $P<10^{-3} \mu$ bar, the gas is fully ionized and the contribution to the number density of electrons by metal species is negligible. The photoionization cross section from $\mathrm{H}(1 \mathrm{~s})$ near the Lyman edge is $6.3 \times 10^{-18} \mathrm{~cm}^{2}$. Since the scale height is less than $10^{9} \mathrm{~cm}$, the gas is optically thin to LyC photons. 


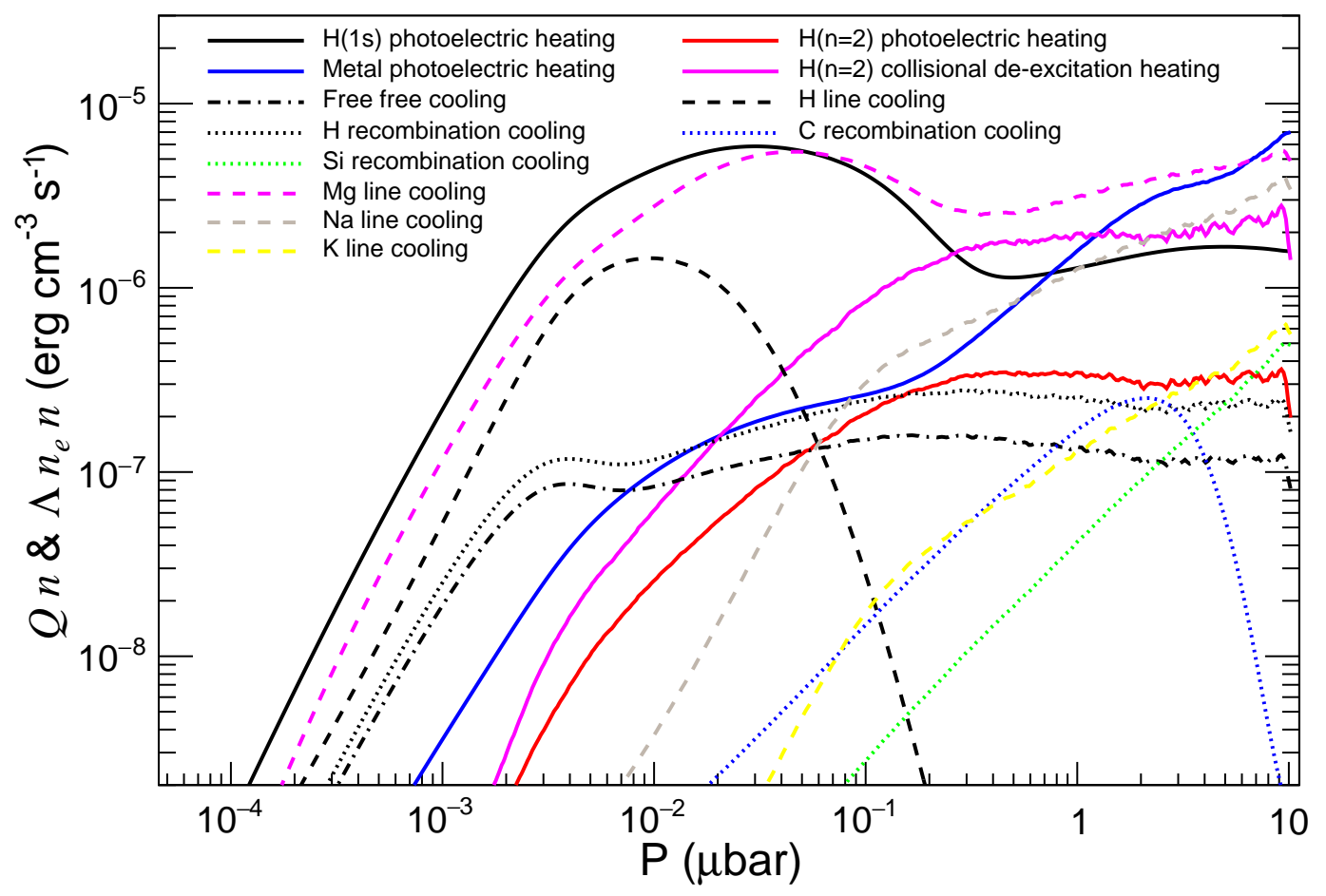

Fig. 2.4. - Major heating $\left(Q n\right.$, solid lines) and cooling $\left(\Lambda n_{e} n\right)$ rates versus pressure $(P)$. The line cooling profiles (dashed lines) present the radiative cooling of Ly $\alpha$ and the metal lines listed in Table 2.3. 


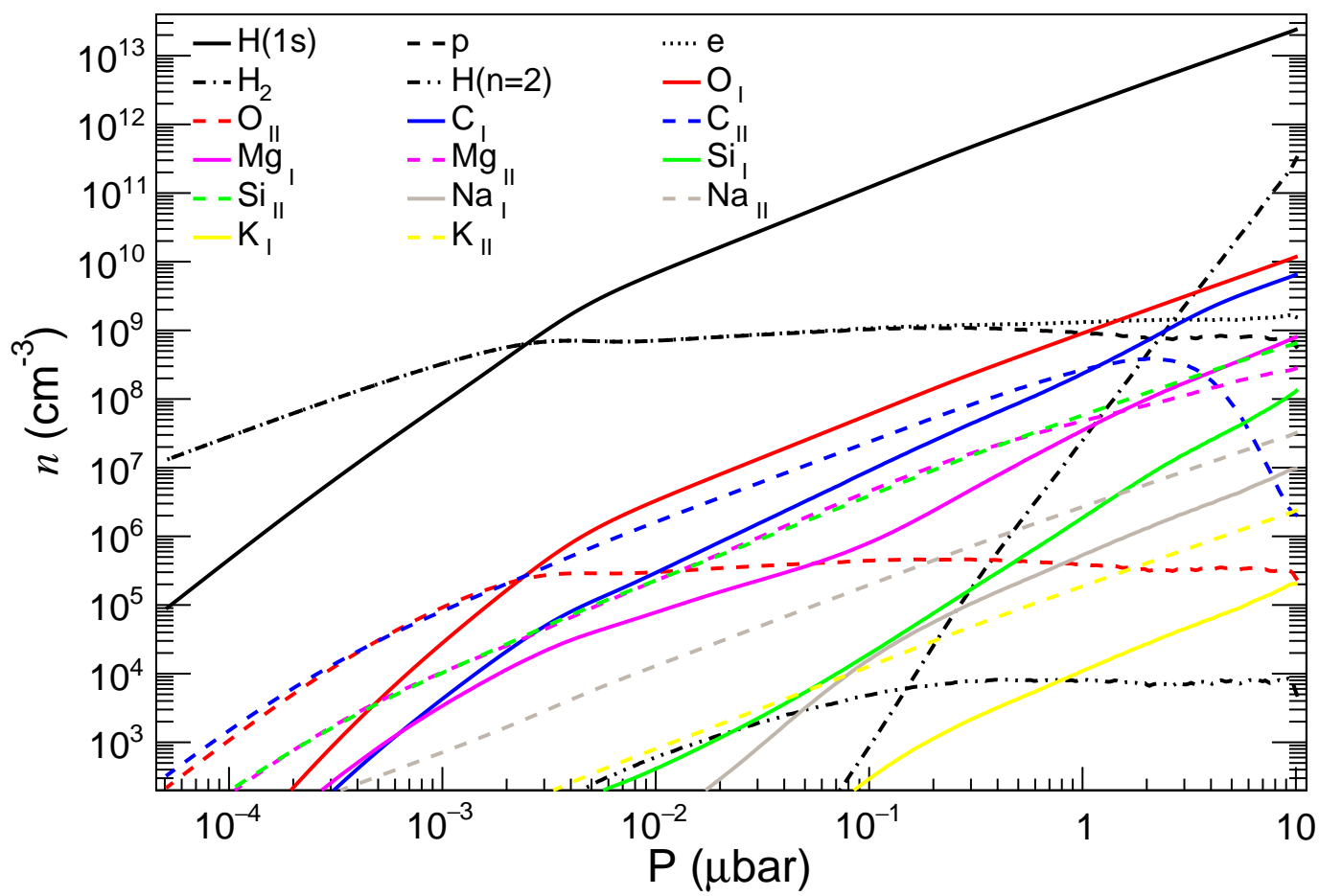

Fig. 2.5. - Number density $(n)$ of main species against the pressure $(P)$. The solid lines present the profiles for neutral atoms and the dashed lines for first ionized ions. The combination of large Ly $\alpha$ intensity $\bar{J}_{L y \alpha} \propto P^{-1}$ and increasing $\mathrm{H}(1 \mathrm{~s})$ density with depth give rise to a nearly flat $\mathrm{H}(2 \ell)$ density around $10^{4} \mathrm{~cm}^{-3}$ over two decades in pressure near the base of thermosphere. The number densities of $\mathrm{Na} I$ and $\mathrm{H}(2 \ell)$ are comparable in the pressure region between $10^{-2} \mu$ bar and $10^{-1} \mu$ bar, where the line center optical depths of $\mathrm{H} \alpha$ and $\mathrm{NaD}$ are near unity (see Figure 2.10). 
In this region $n_{1 s} \approx n_{e}^{2} \alpha_{B} / \Gamma_{1 s} \propto P^{2}$. The gas temperature is set by the balance of $H(1 s)$ photoelectric heating and strong line cooling by Mg and Ly $\alpha$. The cooling rate $n_{\mathrm{Mg}} n_{e} \Lambda_{\mathrm{Mg}} \approx n_{1 s} Q_{1 s} \propto P^{2}$. So the gas temperature is regulated to $T \propto 1 / \log P$ near temperatures $T=9,000-13,000 \mathrm{~K}$, shown in Figure 2.3.

Note that the ionized region of the hydrostatic model at $P \lesssim 3 \times 10^{-4} \mu$ bar may have an unphysically high temperature, as the inclusion of a hydrodynamic outflow and adiabatic cooling may be important in this region, as in the well-studied case of

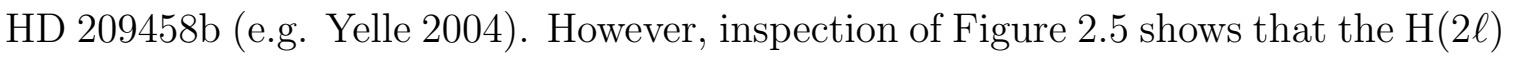
and $\mathrm{Na}$ I densities are negligible in this region, and hence errors in the temperature profile there will not affect the $\mathrm{H} \alpha$ and $\mathrm{Na}$ transit depth.

$\mathrm{H}(1 \mathrm{~s})$ is the main absorber of the stellar LyC flux over the majority of the energy range. Besides $\mathrm{H}(1 \mathrm{~s})$, the 2s-shell of $\mathrm{O}$ I absorbs most photons with energy above $538 \mathrm{eV}$, and $\mathrm{C}$ and $\mathrm{Si}$ are the main absorbers of the photons below $13.6 \mathrm{eV}$ in the atomic layer. Photoelectric heating from ionization of $\mathrm{H}(1 \mathrm{~s})$ contributes the large peak in Figure 2.4 over the pressure range $P=10^{-3}-10^{-1} \mu$ bar. Below that, it continues to be an important source of heating, with absorption of successively higher energy photons with depth, and ionization by secondary electrons becoming important. There is a narrow region near $P=0.1-1 \mu$ bar where heating due to electron impact de-excitation of $\mathrm{H}(2 \ell)$ dominates. Ultimately the heating in this region is due to the $\operatorname{Ly} \alpha$ radiation, which excites the atoms to the $n=2$ state. Near the base, at $P=1-10 \mu$ bar, metal photoelectric heating from ionization of Si, O, and $\mathrm{C}$ dominates the heating. Mg line cooling is dominant over the entire model, with Ly $\alpha$ making an important contribution at $P<10^{-2} \mu$ bar, and Na line cooling below $P=1 \mu$ bar.

Hence near the base of the model, both heating and cooling are controlled by metal 
species, and the temperature is not sensitive to an overall shift in metallicity. Above $P=1 \mu$ bar, the temperature is sensitive to metallicity, and eliminating a single important coolant could make a difference, with $\mathrm{Mg}$ and $\mathrm{Na-poor}$ atmospheres expected to be hotter and more extended. Since this region is important for the $\mathrm{H} \alpha$ and $\mathrm{Na}$ transmission spectrum, the temperature, with it's dependence on the metallicity, may be an important parameter in understanding the transmission spectrum.

Ly $\alpha$ line center optical depth versus height for the fiducial model is also shown in Figure 2.3. Near the base of the atomic layer, $\tau \propto P \propto n(H(1 s))$, since ground state hydrogen is the dominant species. However, above $P=2 \times 10^{-3} \mu$ bar, hydrogen is predominantly ionized, and the optical depth decreases outward roughly as $\tau \propto$ $n(H(1 s)) \propto n_{p}^{2} \propto P^{2}$ in ionization equilibrium. At the outer boundary of the model, $\tau$ drops abruptly to zero due to the $\tau=0$ boundary condition there.

The most abundant species in Figure 2.5 are electrons and protons above $P=$ $3 \times 10^{-3} \mu$ bar, and $\mathrm{H}(1 \mathrm{~s})$ below. Recall that ionization of He is ignored in this paper, and that He is assumed to be neutral and have solar abundance. The electron number density stays roughly constant in the deeper part of the atomic layer because both the ionization and the recombination rates are insensitive to altitude and temperature. The ionization is dominated by the photoionization from $\mathrm{H}(2 \ell)$ in this region and a flat $\mathrm{H}(2 \ell)$ number densities lead to a flat ionization rate (see Figure 2.6). From the near equality $n_{e} \simeq n_{p}$, ionization of hydrogen supplies most of the electrons down to $1 \mu$ bar, and first ionized $\mathrm{Mg}$ and $\mathrm{Si}$ are important below. The ionization state of $\mathrm{O}$ closely follows that of $\mathrm{H}$ because of the very large $\mathrm{O}$ and $\mathrm{H}$ charge exchange rate. The atmosphere becomes opaque to the stellar flux above $10.4 \mathrm{eV}$ due to the absorption by S I and C I. The atmosphere is transparent to stellar flux below the S I ionization threshold throughout the model, which keeps metal species with lower 
ionization energy mostly ionized. The $\mathrm{Mg}$ switches to neutral because its especially small ionization cross section near the ionization threshold. The ionization of $\mathrm{Na}$ I and $\mathrm{K} \mathrm{I}$ is dominated by collisional ionization at the level above $0.1 \mu$ bar. The Na reaches $50 \%$ ionized at $\sim 1$ mbar.

Near the base of the model, the density of $\mathrm{H}_{2}$ rises rapidly, and is only slightly less abundant than $\mathrm{H}(1 \mathrm{~s})$. In a more complete model it is expected that the inclusion of strong molecular cooling due to e.g. $\mathrm{H}_{3}^{+}$and $\mathrm{H}_{2} \mathrm{O}$ would cause the temperature near the base to be even lower and $n_{\mathrm{H}_{2}}$ to be even larger.

The combination of large Ly $\alpha$ intensity $\bar{J}_{L y \alpha} \propto P^{-1}$ (see Figure 2.7) and increasing $\mathrm{H}(1 \mathrm{~s})$ density with depth gives rise to a nearly flat $\mathrm{H}(2 \ell)$ densities around $10^{4} \mathrm{~cm}^{-3}$ over two decades in pressure near the base of atomic layer.

The number densities of $\mathrm{Na} \mathrm{I}$ and $\mathrm{H}(2 \ell)$ are similar in the pressure region between $10^{-2} \mu$ bar and $10^{-1} \mu$ bar. In Section 2.6, it will be shown that $\tau \sim 1$ for $\mathrm{NaD}$ and $\mathrm{H} \alpha$ in this pressure region. This leads to similar transit depths for the $\mathrm{H} \alpha$ and $\mathrm{NaD}$ transmission lines, in agreement with the observations of Cauley et al. (2016) and Wyttenbach et al. (2015).

Shown in Figure 2.6, the photoionization rate from $\mathrm{H}(1 s)$ dominates the $\mathrm{H}$ ionization rate in the top layer of the atmosphere, and becomes constant after the atmosphere becomes optically thick to the LyC photons. The photoionization from $\mathrm{H}(2 \ell)$ takes over for the region $P>10^{-2} \mu$ bar. The rate of charge exchange between $\mathrm{O}$ and $\mathrm{H}$ can be very high, but they almost cancel each other and leave a small net effect. The collisional ionization by secondary $e$ generated by the photoionization of metals $\left(\sum_{m} \Gamma_{m}^{2 \text { nd }} n_{m}\right)$ becomes large at $P>1 \mu$ bar.

The $\mathrm{H}$ radiative recombination cascade process dominates the Ly $\alpha$ photon production throughout the simulation domain. The radiative decay after a thermal $e$ 
collisional excitation from $1 s$ state has a narrow peak in creating Ly $\alpha$ photon near $10^{-2} \mu$ bar. The collisional excitation by $e$ generated by the photoionization of $\mathrm{H}(1 \mathrm{~s})$ $\left(\Gamma_{1 s}^{2 n d} n_{1 s}\right)$ and metals $\left(\sum_{m} \Gamma_{m}^{2 n d} n_{m}\right)$ becomes important in creating Ly $\alpha$ photons near the base of the atomic layer.

The stellar Ly $\alpha$ photons incident through the surface and the photons generated above $10^{-2} \mu$ bar can mostly escape through the top boundary. In contrast, the photons emitted below $0.1 \mu$ bar are mostly absorbed during the resonant scattering processes due to the high optical depth. The Ly $\alpha$ flux at the bottom boundary is about 10 erg $\mathrm{cm}^{-2} \mathrm{~s}^{-1}$.

The total LyC flux absorbed inside the simulation domain, is $F_{\mathrm{LyC}}=1.4 \times$ $10^{4} \mathrm{erg} \mathrm{cm}^{-2} \mathrm{~s}^{-1}$. Besides the $2.0 \times 10^{4} \mathrm{erg}^{-2} \mathrm{sm}^{-1}$ stellar Ly $\alpha$ flux that mostly

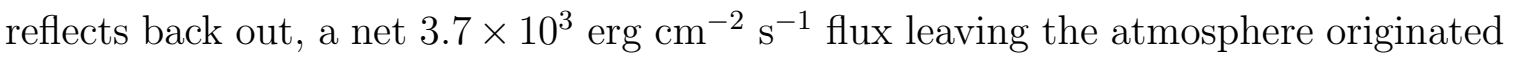
from the Ly $\alpha$ emission inside the atmosphere. In comparison, integrating the source function in Equation 2.35 over height, solid angle and frequency, the total column Ly $\alpha$ internal emission is $1.3 \times 10^{4} \mathrm{erg} \mathrm{cm}^{-2} \mathrm{~s}^{-1}$. The large internal Ly $\alpha$ emission rate is because the Ly $\alpha$ photons generated deep in the atmosphere are absorbed locally, rather than the heating efficiency is very high.

The local balance of sources and sinks in region below $0.2 \mu$ bar, as well as the small flux hitting the bottom boundary, imply that the Ly $\alpha$ photons in this region reach local equilibrium between sources and sinks. Because the photoionization of an $\mathrm{H}(2 \ell)$ is followed by a radiative recombination cascade, emitting another Ly $\alpha$ photon, these two processes taken together can be thought of not as sources or sinks, but as a redistribution in photon energy. The absorption of a photon on the wing, and its subsequent re-emission at line center make it harder for the photon to escape the atmosphere. The most important remaining "real" photon sources are excitation by 
secondary $e$, and radiative recombination cascade of a $p$ which was collisionally ionized by high energy photoelectron. The "real" photon sinks are collisional de-excitation, photoionization of metals by Ly $\alpha$, and 2 photon decay.

A breakdown of line profile weighted mean intensity, $\bar{J}_{L y \alpha}$, in terms of the different sources is given in Figure 2.7. The external Ly $\alpha$ directly from star dominates above $0.05 \mu$ bar and the large intensity stays roughly constant. Because the incident stellar Ly $\alpha$ is peaked at the frequency that is more than 5 Doppler widths away from the line center, the cross section to these photons in the Lorentzian wing is about $10^{5}$ smaller than the cross section in the line center. Most stellar Ly $\alpha$ photons can directly penetrate to the $P \sim 10^{-2} \mu$ bar level, which leads to a roughly constant intensity above this layer. Radiative recombination cascades are more important below that, with secondary $e$ excitation becoming the second largest source deeper than $1 \mu$ bar.

The Ly $\alpha$ mean intensity near the base can be estimated by assuming a local balance of frequency-integrated sources and sinks, giving $J \simeq S L$. Here $J$ is the frequency integrated mean intensity, $S$ is the frequency integrated source function, and $L$ is the total path length traversed by the photon before it is destroyed. The photon source is insensitive to altitude as shown in Figure 2.6. For a sink given by true absorption due to photoionization of metals, the mean intensity is

$$
J \simeq S L=\frac{S}{n_{m} \sigma_{m, \mathrm{pi}}\left(\nu_{L y \alpha}\right)}
$$

The photoionization cross section of metals by Ly $\alpha$ photons, $\sigma_{m, \mathrm{pi}}\left(\nu_{L y \alpha}\right)$, is independent of altitude and frequency. The number densities of Mg I, Si I, Na I, and K I scale proportional or slightly steeper than $P$. As a result, the $J \propto P^{-1}$. Next consider 
collisional de-excitation and 2 photon decay, for which

$$
\begin{aligned}
J & \simeq S \epsilon^{-1} l_{\mathrm{mfp}} \\
& =S\left(\frac{3 A_{2 p \rightarrow 1 s}}{4 n_{e} C_{2 \rightarrow 1 s}(T)+A_{2 s \rightarrow 1 s}}\right)\left(\frac{1}{n_{1 s} \sigma_{1 s}\left(\nu_{0}\right)}\right)
\end{aligned}
$$

where $\epsilon$ is the probability of excitation not followed by de-excitation, and $l_{\mathrm{mfp}}$ is the line center mean free path. Because $n_{e}, C_{2 \rightarrow 1 s}(T)$ are insensitive to altitude, $J \propto P^{-1}$ in this case also.

To show the reliability of the approximations given in Equation 2.14, which are used in the hydrostatic model, the results for the $\mathrm{H}(1 s)$ number density and temperature were plugged back to the full hydrogen level population code described in Section 2.2. Figure 2.8 compares the approximate result and the full calculation for the $n_{e}, n_{2 s}$, and $n_{2 p}$. The approximation holds for the whole simulation region. The $n_{2 s}$ and $n_{2 p}$ obtained from both methods are almost identical except the approximate method slightly underestimates the $n_{2 s}$ at the very top of the atmosphere, due to the fact that the contribution from collisional excitation is not completely negligible there. The $n_{e}$ obtained from the hydrogen level population calculation is slightly larger, because recombinations are only included up to $n=6$ there, while the case $\mathrm{B}$ recombination rate is summed over all levels.

The number density of $\mathrm{H}(n=3)$ obtained from the level population is also shown in the Figure 2.8. Because an optically thin stellar $\mathrm{H} \alpha$ intensity is applied and Ly $\beta$ radiation transfer is not carefully considered, the number density of $\mathrm{H}(n=3)$ shown here is only a roughly estimate. Nevertheless, the low number density indicates that the Paschen series absorption features are unlikely to be observed. 


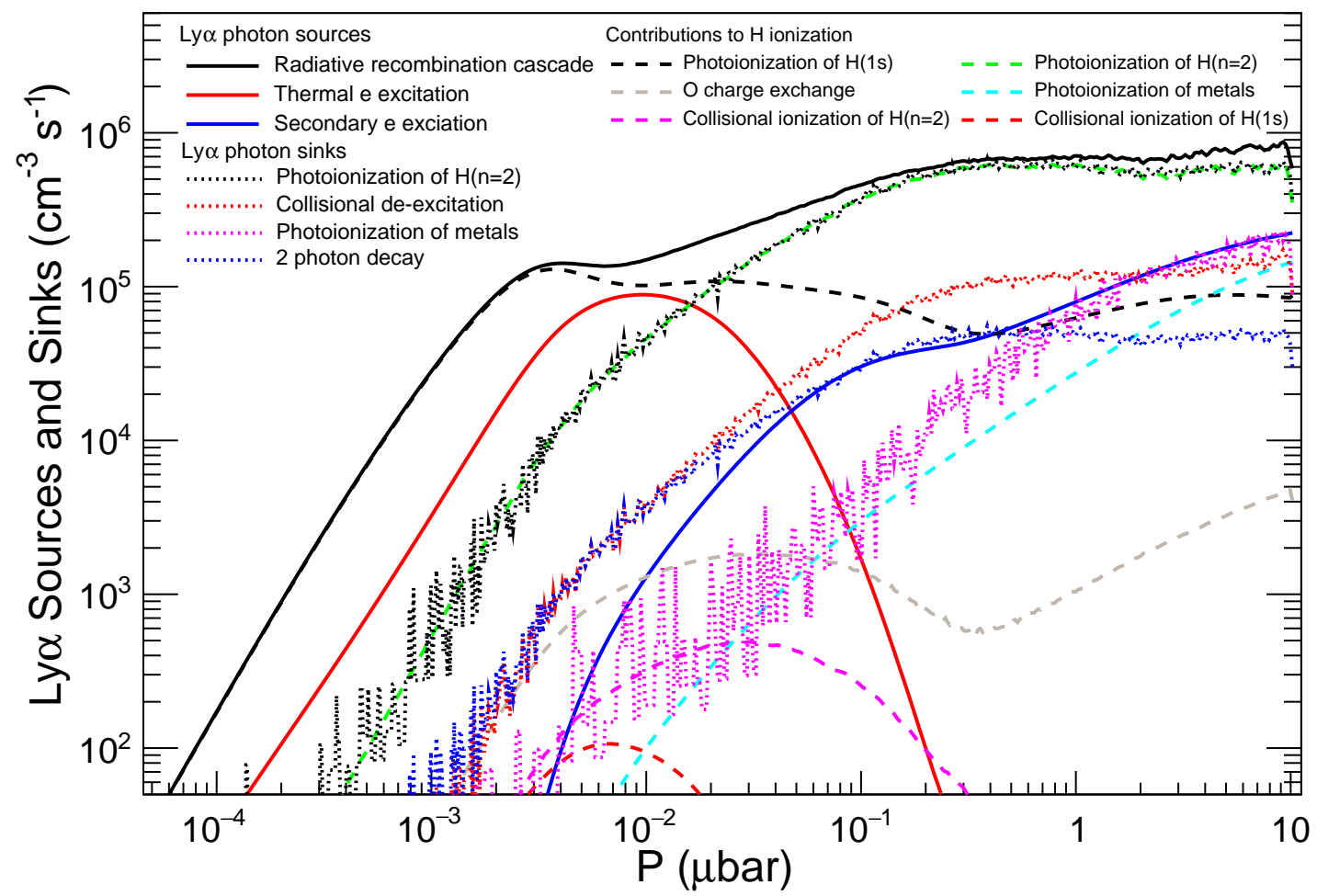

Fig. 2.6. - Ly $\alpha$ photon sources, sinks, and $\mathrm{H}$ ionization rates per unit volume as a function of pressure $(P)$. Each Ly $\alpha$ photon source in Equation 2.35 is plotted with a solid line. The secondary $e$ excitation stands for the collisional excitation by $e$ generated by the photoionization of $\mathrm{H}(1 s)\left(\Gamma_{1 s}^{2 n d} n_{1 s}\right)$ and metals $\left(\sum_{m} \Gamma_{m}^{2 n d} n_{m}\right)$. The sink rates are the output from the Ly $\alpha$ Monte Carlo simulation. The process that a Ly $\alpha$ photon photoionizes a low ionization potential metal atom is referred as the photoionization of metals under the Ly $\alpha$ photon sinks section. A breakdown of the $\mathrm{H}$ radiative recombination cascade rate into individual $\mathrm{H}$ ionization processes based on Equation 2.21 is also plotted. The output rate of photoionization of $\mathrm{H}(2 \ell)$ in the Ly $\alpha$ Monte Carlo simulation as a photon sink recovers the rate of the same process as a $\mathrm{H}$ ionization given by the ionization equation. $\mathrm{O}$ charge exchange stands for the difference between recombination of an $\mathrm{O}$ II and ionization an $\mathrm{O}$ I by charge exchange with $\mathrm{H}\left(k_{\mathrm{rec}}^{(\mathrm{O})} n_{\mathrm{O} \text { II }} n_{1 s}-k_{\text {ion }}^{(\mathrm{O})} n_{\mathrm{OI}} n_{p}\right)$. The photoionization of metals listed here stands for the $\mathrm{H}$ collisional ionization by $e$ generated by the photoionization of metals $\left(\sum_{m} \Gamma_{m}^{2 \text { nd }} n_{m}\right)$. 


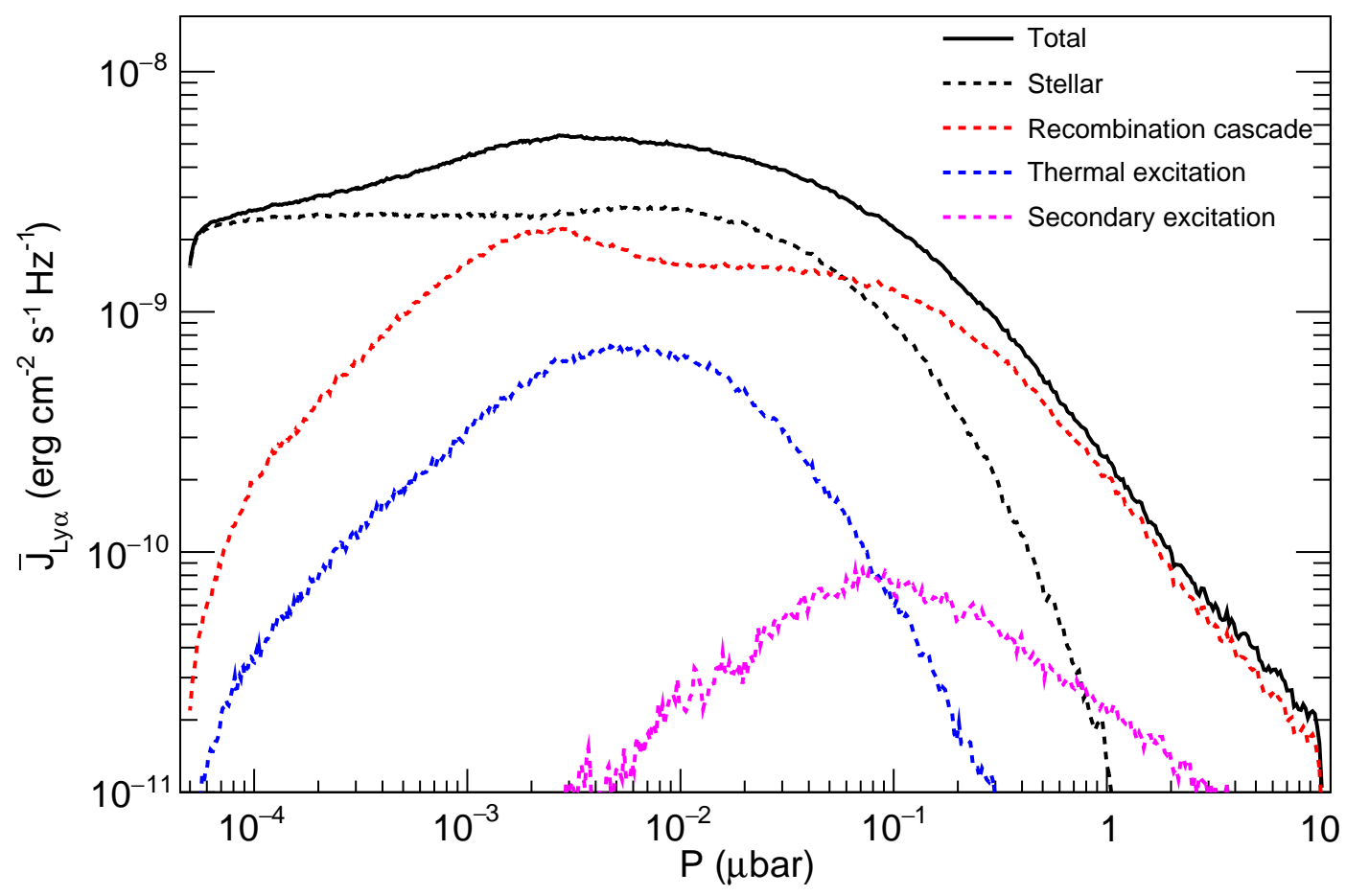

Fig. 2.7.- Line profile weighted mean intensity $\bar{J}_{L y \alpha}$ from different initial Ly $\alpha$ photon generation mechanisms against the pressure $(P)$. The large $\bar{J}_{L y \alpha}$ stays roughly constant deep into the atmosphere and $\bar{J}_{L y \alpha} \propto P^{-1}$ near the base of the atomic layer. Both Ly $\alpha$ photons created inside the atmosphere and incident from the star are important in the $\mathrm{H} \alpha$ line formation region.

\subsection{Transmission Spectrum}

\subsection{1 $\mathbf{H} \alpha$ Transmission Spectrum}

From the discussion in Section 2.4, the Ly $\alpha$ intensity is small in the molecular layer at $r<R_{b}$. As a result, the $\mathrm{H}(2 \ell)$ density there is small, and the region between $R_{p}$ and $R_{b}$ is transparent to $\mathrm{H} \alpha$.

Figure 2.9 shows the model $\mathrm{H} \alpha$ transmission spectrum and the data from Cauley et al. $(2015,2016)$. The fiducial model discussed in Section 2.5 is given by the black line labeled " $\xi=1, P_{\mathrm{b}}=10 \mu$ bar". Given the noise in the data, the fiducial model 


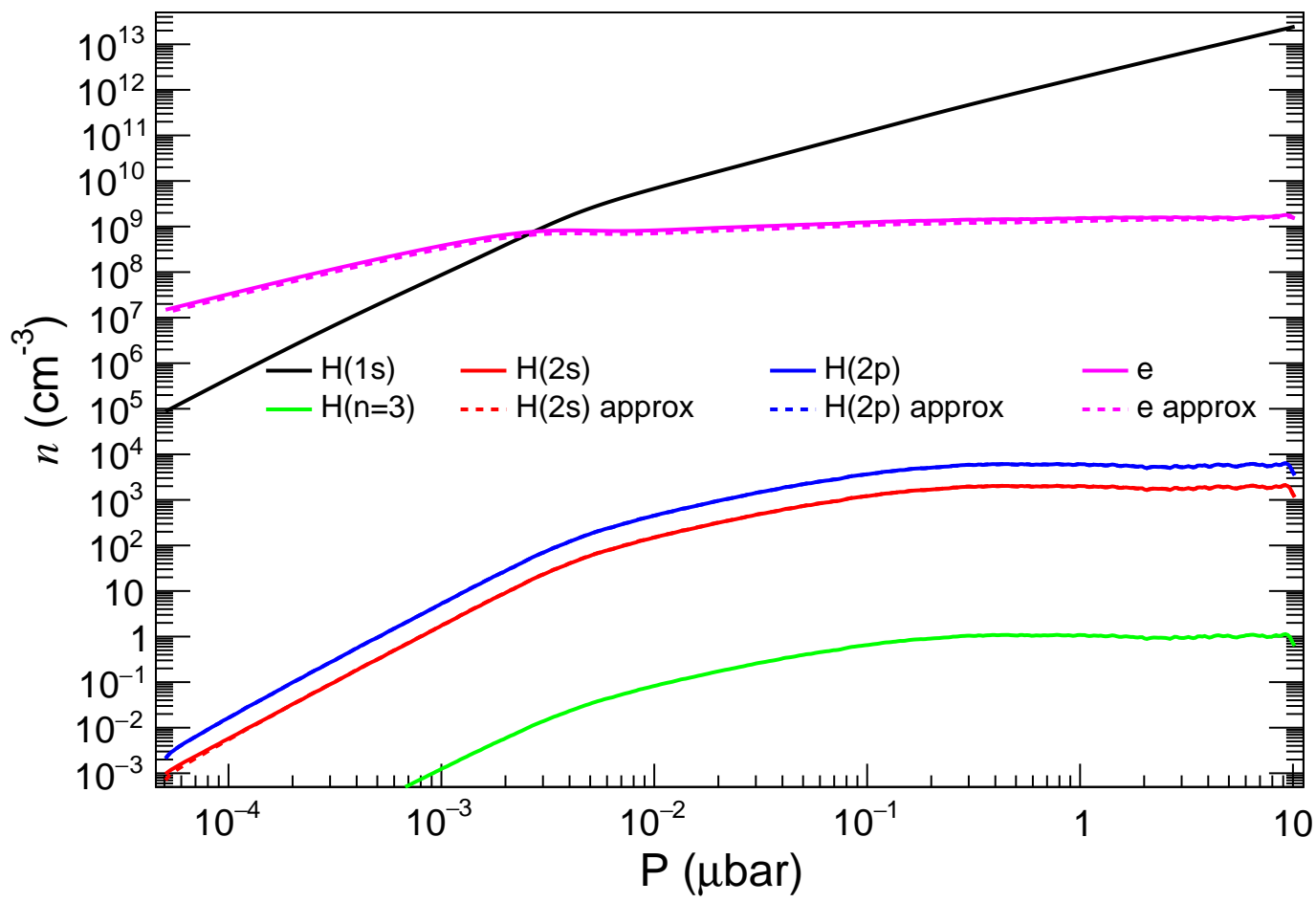

Fig. 2.8. - Comparison of the hydrogen sub-states and electron number densities $(n)$ obtained from the full hydrogen level population code (solid line) and the approximation in Equation 2.14 (dashed line), which mostly overlaps with the solid lines. A rough estimate of the $\mathrm{H}(n=3)$ number density is also shown in green.

is in broad agreement for both the line center absorption depth and the line width. The wavelengths have been corrected for the index of refraction of air at "standard condition", $n_{\mathrm{H} \alpha}=1.0002762$, according to Cox (2000). The plot also shows the effect of a different LyC flux, as denoted by lines with a different value of the factor $\xi$ and the metallicity, which will be discussed in the Section 2.6.4 and 2.6.5, respectively.

The base pressure, $P_{\mathrm{b}}$, is not self-consistently determined in this study. In order to investigate the dependence of the transmission spectrum on this parameter, the blue solid line labeled " $\xi=1, P_{\mathrm{b}}=1 \mu$ bar" shows a model with the base of the atomic layer at $P_{b}=1 \mu$ bar. The line center transit depth is smaller by $\simeq 20 \%$ for $P_{b}=1 \mu$ bar. 


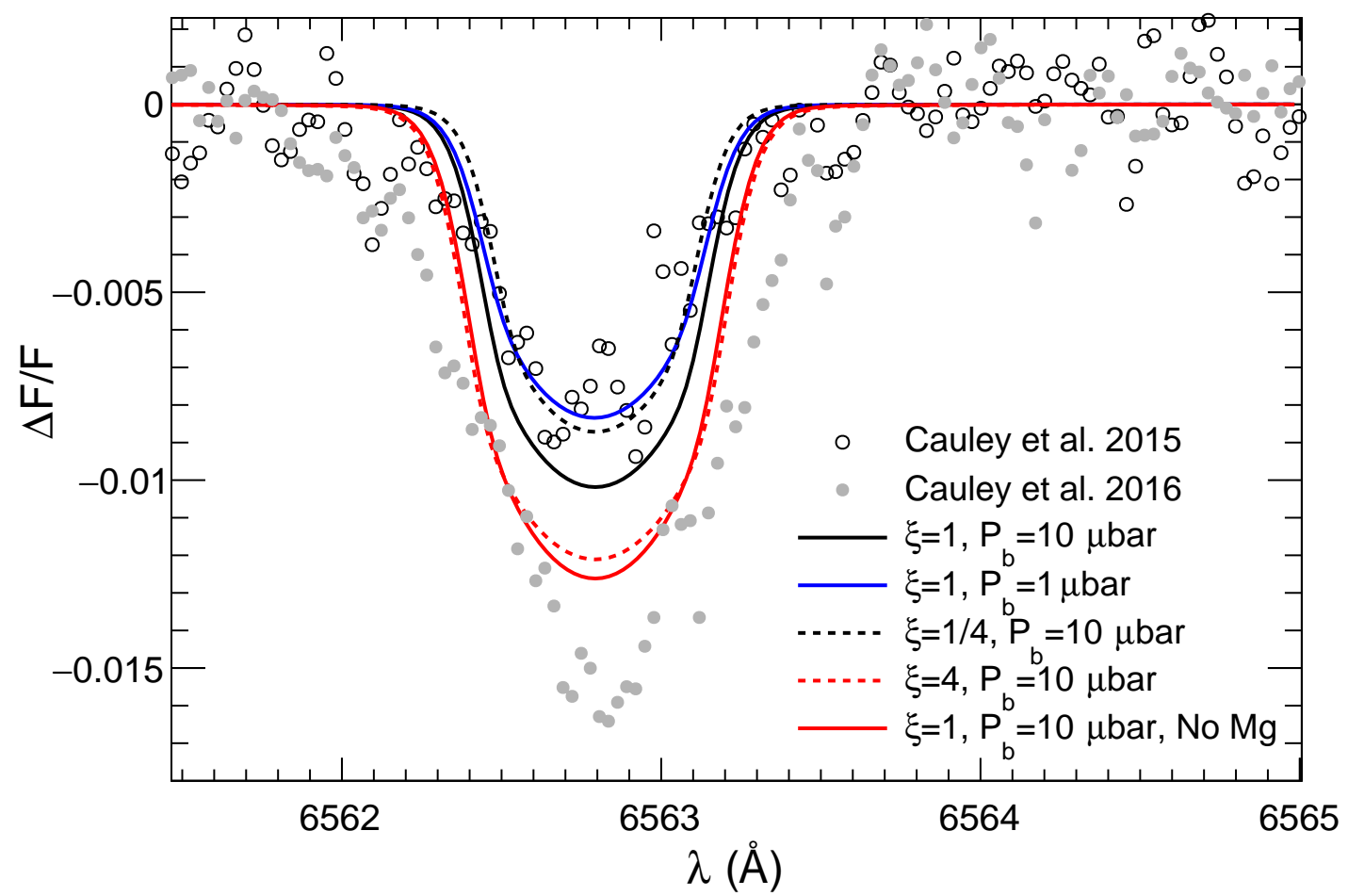

Fig. 2.9.- $\mathrm{H} \alpha$ transmission spectrum. The black solid line shows the transmission spectrum of the fiducial model. The blue solid line shows the model with atomic layer base pressure $P_{b}=1 \mu$ bar. Black and red dashed lines show the model with stellar LyC multiplier factor $\xi=1 / 4$ and $\xi=4$ respectively. The red solid line shows the model without $\mathrm{Mg}$. Circles in the plot are observational data from Cauley et al. (2015, 2016).

Changing this boundary causes only small changes the atmosphere properties, so the $\mathrm{H} \alpha$ becomes optically thick at roughly the same pressure. However, because the scale height between $1 \mu$ bar and $10 \mu$ bar significantly decreases after switching to lower temperature and larger mean molecular weight, the radius in the atomic layer that corresponds to the same pressure becomes smaller, which leads to a smaller transit depth. Although we do not expect the transition from atomic layer to molecular layer to be as high up as $1 \mu$ bar based on Figure 2.5, a more physical molecular model is required to produce a more precise transmission spectrum. 


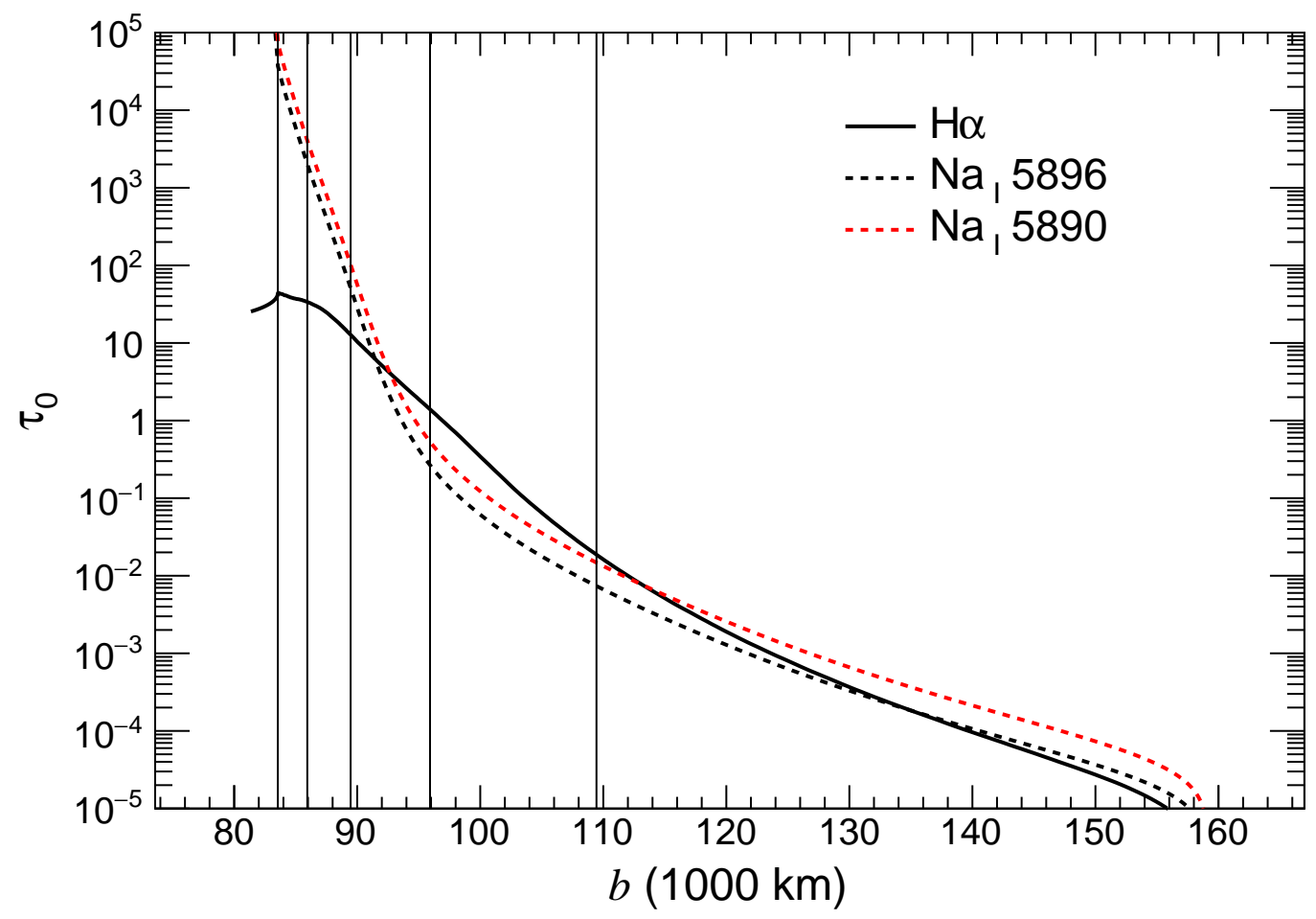

Fig. 2.10.- $\mathrm{H} \alpha$ and $\mathrm{NaD}$ doublet line center optical depth $\left(\tau_{0}\right)$ versus impact parameter $(b)$. The vertical solid lines show the location of pressure levels $10 \mu \mathrm{bar}, 1 \mu \mathrm{bar}$, $0.1 \mu$ bar, $0.01 \mu$ bar, and $0.001 \mu$ bar from left to right.

Figure 2.10 shows the line center optical depth of the fiducial model versus impact parameter $b$. The $\mathrm{H} \alpha$ line center optical depth reaches the maximum value $\sim 40$ for $b=R_{\mathrm{b}}$, the base of the atomic layer. Although the optical depth of $\mathrm{H} \alpha$ slightly decreases inward, due to the (assumed) transparent molecular layer, the optical depth is still much larger than 1 all the way to the continuum radius $b=R_{\mathrm{p}}$. Lecavelier Des Etangs et al. (2008) showed that the optical depth at the effective radius is $\tau_{e q} \simeq 0.56^{1}$, and is not sensitive to the details of the atmospheric structure. In the

\footnotetext{
${ }^{1}$ With the approximation of the uniform mixing ratio and isothermal thin atmosphere, the integral in Equation 1.5 may be expanded in a series as $\int_{0}^{\infty} \mathrm{d} u\left(1-e^{-\beta e^{-u}}\right) \simeq \ln (\beta)+\gamma+\mathcal{O}\left(\beta^{-2}\right)$, where $\gamma \simeq 0.577$ is the Euler-Mascheroni constant and $\beta \simeq n\left(R_{p}\right) \sigma \sqrt{2 \pi R_{p} H}$. This formula is valid for $\beta \gg 1$. This expansion then gives $\tau_{\text {eq }}=e^{-\gamma} \simeq 0.561$, in good agreement with Lecavelier Des Etangs
} 


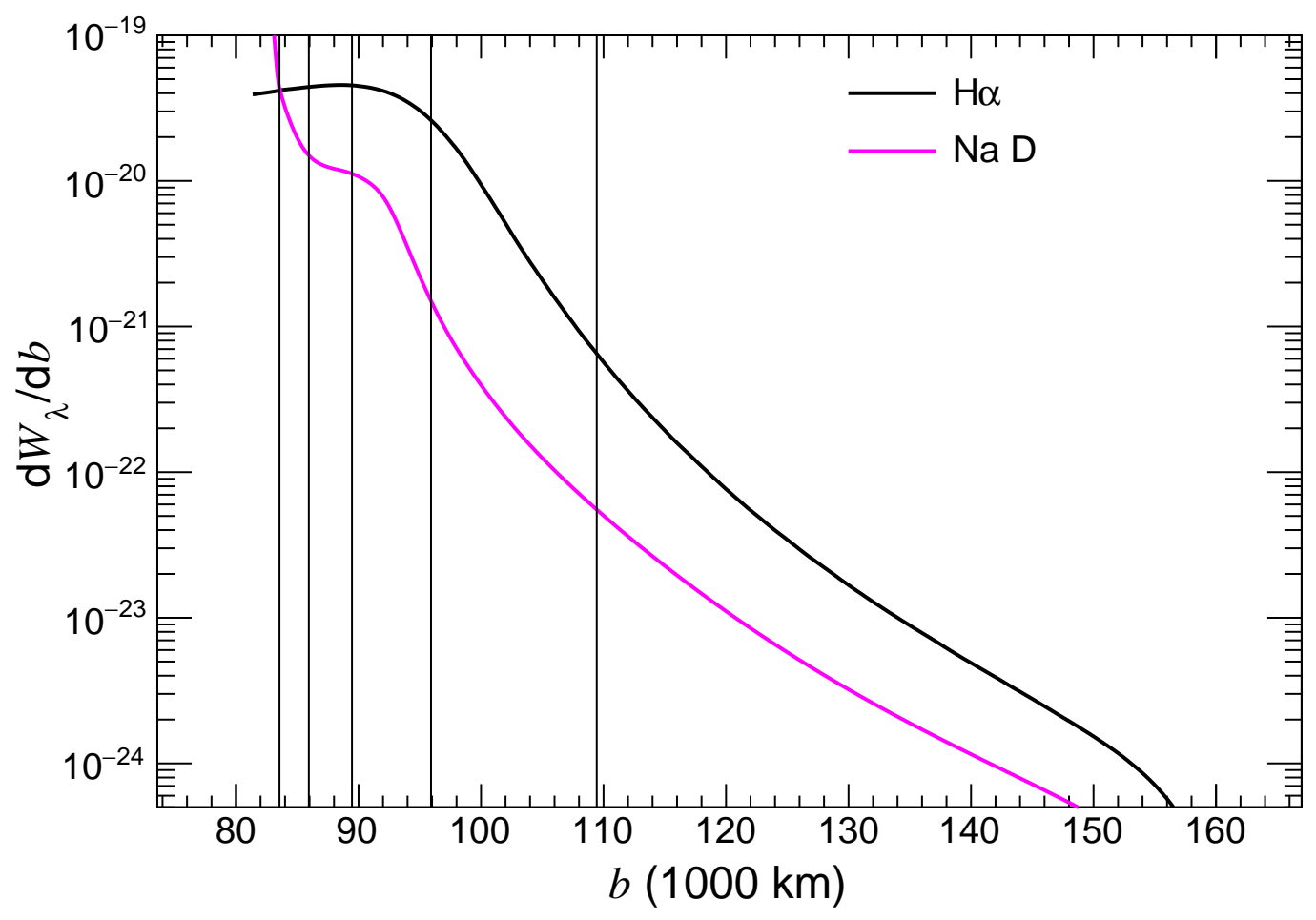

Fig. 2.11. - $\mathrm{H} \alpha$ and $\mathrm{NaD}$ transmission spectrum equivalent width per unit impact parameter $\left(\mathrm{d} W_{\lambda} / \mathrm{d} b\right)$ (defined in Equation 2.39) versus b. The contribution to $W_{\lambda}$ by $\mathrm{NaD}$ doublet are summed together. The vertical solid lines show the location of $10 \mu$ bar, $1 \mu$ bar, $0.1 \mu$ bar, $0.01 \mu$ bar, and $0.001 \mu$ bar from left to right.

fiducial model, the effective radius is $9.87 \times 10^{9} \mathrm{~cm}$, corresponding to an $\mathrm{H} \alpha$ optical depth $\tau=0.54$ and pressure $P=5.1 \times 10^{-3} \mu$ bar. The optical depth drops to below $\sim 10^{-2}$ at a pressure $10^{-3} \mu$ bar, which means the contribution to $\mathrm{H} \alpha$ absorption from the atmosphere above this level is small.

To indicate the vertical distribution of $\mathrm{H} \alpha$ and $\mathrm{NaD}$ absorption by the atmosphere, Figure 2.11 shows the equivalent width contributed by an annulus of atmosphere with et al. (2008). 
radius $b$, defined as

$$
\frac{\mathrm{d} W_{\lambda}}{\mathrm{d} b}=\frac{2 b}{R_{\star}^{2}-R_{p}^{2}} \int\left(1-e^{-\tau_{l, \nu}(b)}\right) \mathrm{d} \lambda .
$$

For impact parameters in the range $R_{\mathrm{p}}<b<R_{\mathrm{b}}$, which go through the molecular layer, the $\mathrm{H} \alpha$ line center optical depth is $\simeq 40$, and the absorption in the Lorentzian damping wing is negligible. Therefore, the base of the atomic layer is in the flat portion of the curve of growth (Draine 2011). The contribution to the equivalent width decreases slowly inward in this part of the atmosphere because of the smaller annulus radius and the lower temperature. It is also shown that the $\mathrm{H} \alpha$ absorption is dominated by impact parameters below $P \sim 5 \times 10^{-3} \mu$ bar, where the line center optical depth is larger than 0.5 .

\subsubsection{NaD Transmission Spectrum}

Unlike $\mathrm{H} \alpha$, the $\mathrm{NaD}$ doublet lines are absorbed by ground state $\mathrm{Na}$, which has high density deep in the atmosphere. The molecular layer is not treated in detail here, rather a simple model with constant (solar abundance) mixing ratio and temperature $T=1140 \mathrm{~K}$ is used. For simplicity, $\mathrm{Na}$ is assumed to be neutral in the molecular layer, even though $\mathrm{Na}$ I is expected to have slightly smaller abundance than Na II until $\sim$ mbar pressure. Similar to $\operatorname{Ly} \alpha, \mathrm{NaD}$ photons undergo a resonant scattering process in the atmosphere, at least at pressures sufficiently low that collisional deexcitation and collisional broadening are negligible. An accurate model of the NaD transmission spectrum requires treatment of resonant scattering by $\mathrm{Na} \mathrm{I}$, as well as true absorption and emission by the atmosphere, which is beyond the scope of this

paper. Instead, as was done for $\mathrm{H} \alpha$ in this work, a simple $e^{-\tau_{l, \nu}(b)}$ absorption will be used to compute the transmission spectrum. Because the line wings of the NaD 


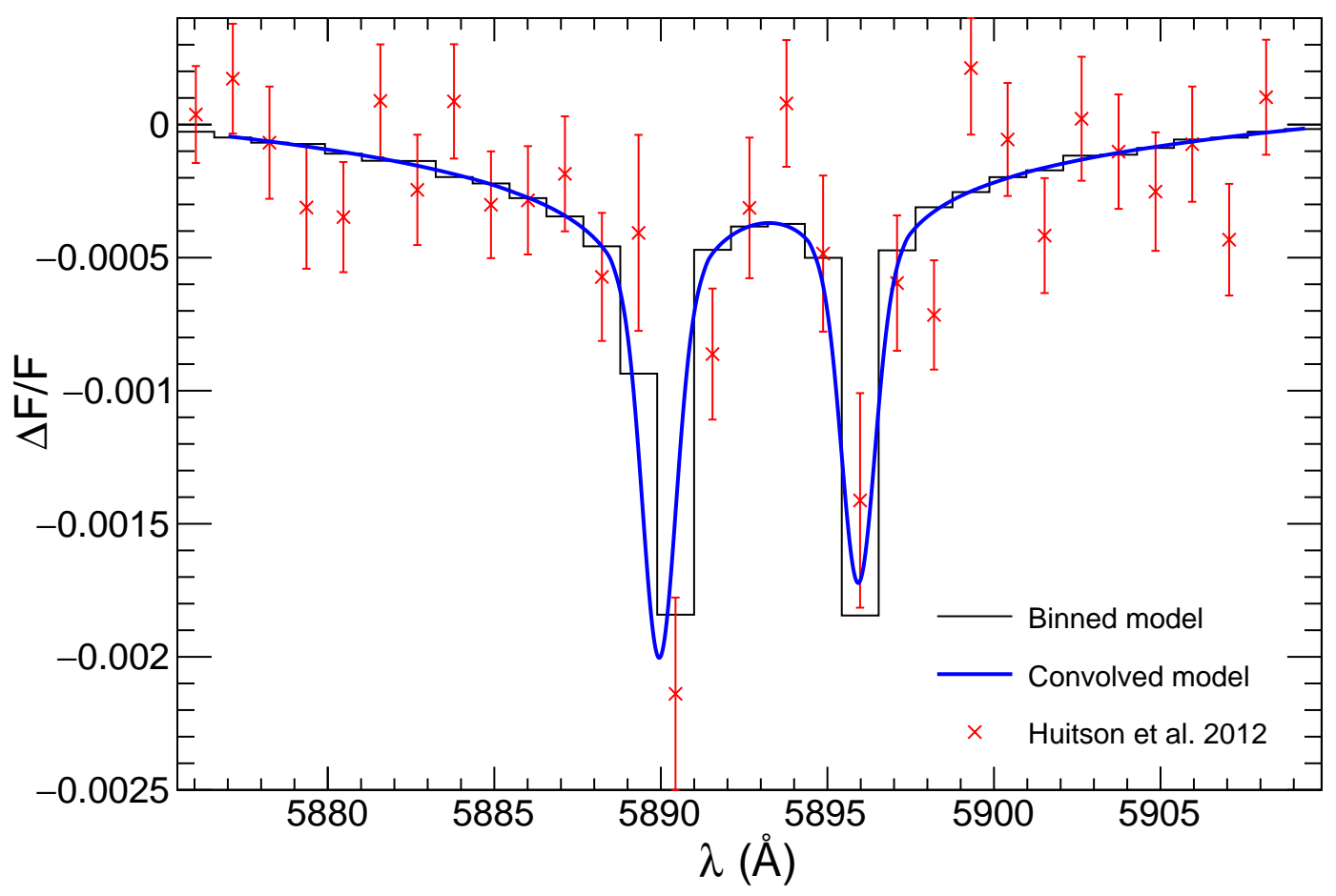

Fig. 2.12. - Comparison of the $\mathrm{NaD}$ doublet transmission spectrum observed by Huitson et al. (2012) to the transmission spectrum of the fiducial model $\left(\xi=1, P_{b}=\right.$ $10 \mu$ bar $)$. The histogram shown as a black line is the modeled spectrum binned to the instrument resolution. The blue curve shows the result of the modeled spectrum convolved with a Gaussian profile with FWHM matching the instrument resolution.

doublet are overlapping, $\tau_{l, \nu}(b)$ uses the sum of the cross sections for each line of the doublet, evaluated at frequency $\nu$.

Figure 2.10 shows that the optical depths of the $\mathrm{NaD}$ doublet lines reach $\tau \sim 0.5$ at $P \sim 10^{-2} \mu$ bar, comparable to that of $\mathrm{H} \alpha$, agreeing with the inference made previously based on the similar transit depth of $\mathrm{H} \alpha$ and $\mathrm{NaD}$. At this altitude, the temperature is $\sim 8000 \mathrm{~K}$, comparable to the analytic estimate using the difference between the NaD doublet transit depths discussed in Section 2.1. The optical depth of the NaD doublet become much larger than unity below $1 \mu$ bar. Thus NaD absorption 


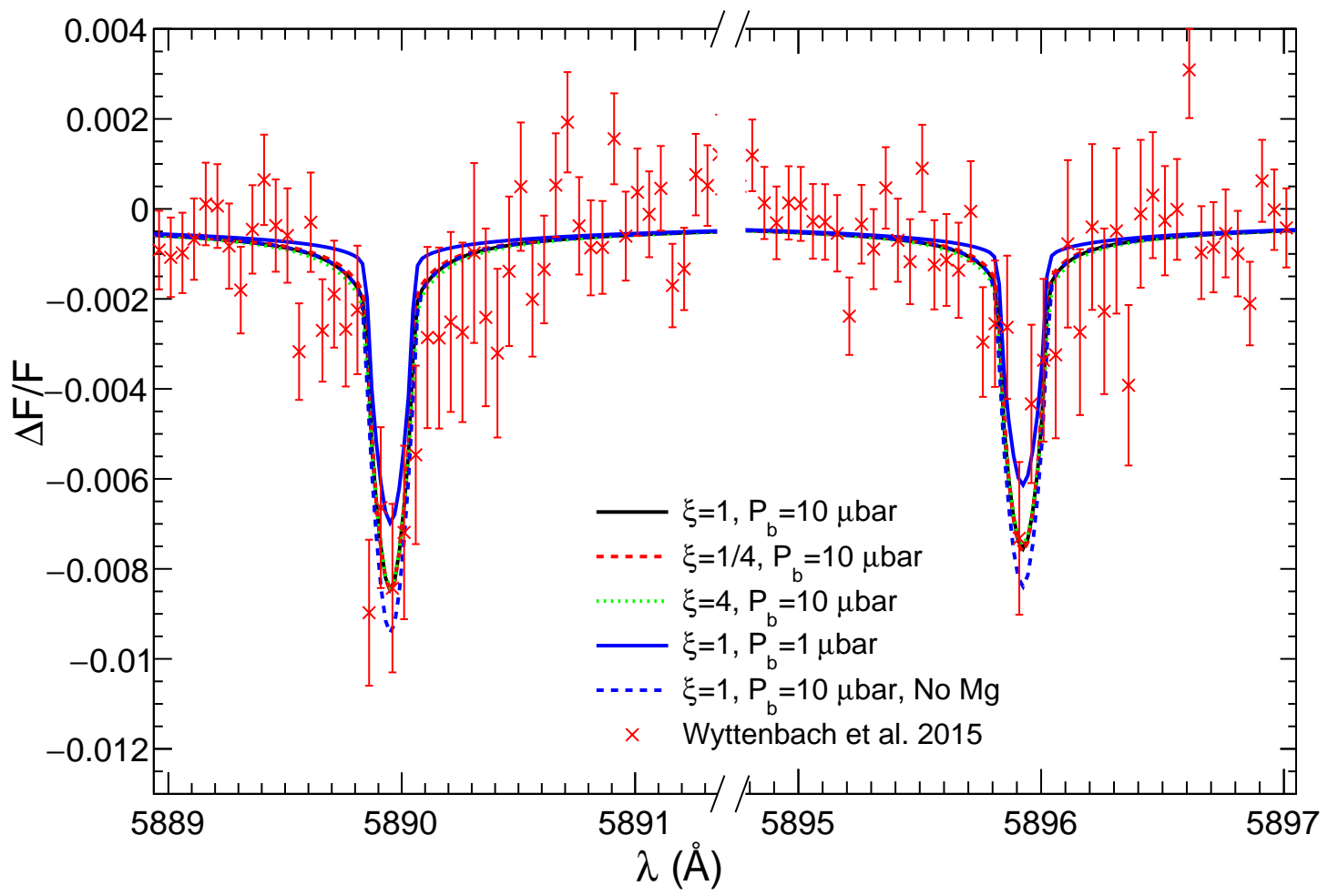

Fig. 2.13. - Comparison of the $\mathrm{NaD}$ doublet transmission spectrum observed by Wyttenbach et al. (2015) binned by $5 \times$, to the calculated transmission spectrum of the fiducial model, as well as models with different atomic layer base pressures $P_{b}$, LyC boost factor $\xi$ and metallicity. Note the break in the x-axis. The spectra of the three models with $P_{b}=10 \mu$ bar and solar abundance are nearly overlap on each other.

by the atmosphere near the base of the atomic layer is in the damped portion of the curve of growth, which explains the large contribution to the equivalent width shown in Figure 2.11. However, because of the slow transit depth variation with frequency on the damping wing, it is difficult to distinguish the $\mathrm{NaD}$ damping wings from possible additional sources of continuum opacity due to the observational error bars. The presence of clouds or hazes would further complicate the detection of this lower portion of the atmosphere, in spite of its large equivalent width contribution.

Figure 2.12 compares the observed NaD transmission spectrum from Huitson et al. 
(2012) and the fiducial model. The wavelength has been corrected for the index of refraction of air, $n_{\mathrm{NaD}}=1.0002771$ according to Cox $(2000)$. The spectral resolution of the Space Telescope Imaging Spectrograph (STIS) G750M grating aboard the HST used in this observation is $\sim 6$ times broader than the full width at half maximum (FWHM) of each line in the NaD doublet. Two methods are used to compare the model spectrum with the low spectral resolution observation. The first method is described in Huitson et al. (2012), and is shown as a black histogram. The model spectrum is binned to the STIS instrument resolution, 2 pixels, since the G750M grating gives a resolution $\sim 2$ pixels at $5893 \AA$. Care is required since the absorption depth of the binned spectrum near each line center depends on the wavelength range used for binning. In view of this, the second method, shown as a blue solid curve, convolves the model spectrum with a Gaussian profile with FWHM matching the instrument resolution (2 pixel widths). For both methods, to imitate the process of normalization to the continuum outside the regions of interest which been done in observational data reducing, the $\mathrm{Na}$ absorption depth at $5912 \AA$ is subtracted out and treated as the continuum.

Figure 2.13 compares the high resolution $\mathrm{NaD}$ transmission spectrum observed by Wyttenbach et al. (2015) with the fiducial model, as well as models with different atomic layer base pressures $P_{b}, \xi$ and metallicity, equivalent to the models shown in Figure 2.9. In comparing to the $\mathrm{H} \alpha$ transmission spectrum, recall that the line width of $\mathrm{Na}$ is narrower as compared to $\mathrm{H} \alpha$ due to the larger mean atomic weight of $\mathrm{Na}$. To reduce the noise, the data plotted are binned by $5 \times$. The resulting $0.05 \AA$ bin width is equal to the FWHM of the average spectrograph line spread function, and is $\sim 3$ times narrower than the NaD FWHM. On top of the $2.3 \mathrm{~km} \mathrm{~s}^{-1}$ shift to the red which accounts for the systemic velocity, the data were shifted by $10 \mathrm{~km} \mathrm{~s}^{-1}$ to the red 
to cancel the observed blueshift from an unknown source described in Wyttenbach et al. (2015). Similar to the treatment in Figure 2.12, the correction from the index of refraction and continuum flux are made to the simulated spectrum. No binning or convolving is required because the spectral features are well resolved.

The line center absorption depths generated by the models agree with the NaD spectrum in both observations roughly to the level of the observational error bars. Note that there is a strong absorption feature on the red side of the line center which cannot be explained by the model.

\subsubsection{Retrieval of the Temperature Profile from NaD Trans- mission Spectra}

To measure the temperature versus altitude profile from the NaD transmission spectrum, $r(\lambda)$, Lecavelier Des Etangs et al. (2008) applied the analytic model

$$
r(\lambda)=H \ln (\sigma(\lambda))+\text { constant }
$$

which is derived for a plane-parallel isothermal atmosphere with uniform mixing ratio of $\mathrm{Na}$ I and scale height $H=k_{\mathrm{B}} T / \mu m_{p} g$. Here $\sigma(\lambda)$ is the summed cross section from each line of the $\mathrm{NaD}$ doublet, and a Voigt profile at the local temperature is used. The wavelength-independent constant term is determined by the radius at continuum wavelengths. If the temperature and abundance vary slowly with altitude, an approximate scale height $H$ of the atmosphere at a certain radius can be derived from $H=(\mathrm{d} r / \mathrm{d} \lambda) /(\mathrm{d} \ln \sigma / \mathrm{d} \lambda)$. Then, applying a mean molecular weight $\mu$, the local temperature $T(r)$ at $r(\lambda)$ can be computed from the fitted value of $H$.

Methods similar to this were applied by Huitson et al. (2012) and Wyttenbach 
et al. (2015) to measure the upper atmosphere temperature from their observed $\mathrm{Na}$ transmission spectra. The atmosphere was assumed to be molecular with $\mu=2.3$ in both studies. To decrease the uncertainty of the temperature measurement due to noise in the observed $r(\lambda)$ profile, Huitson et al. (2012) broke the spectrum into small wavelength intervals, and fit $r(\lambda)$ in each interval by varying $H$ and the constant. Wyttenbach et al. (2015) also broke the spectrum into intervals, and fit for $H$ in each spectral region, but with a fixed value for the constant term in Equation 2.40 in each interval. As a result, if connecting the fitting curves from separate wavelength ranges together, the joined curve is not continuous in both the slope and value of the transit depth at the boundary between adjacent wavelength ranges.

The measured temperatures near line center in both studies are more than a factor of 2 lower than the model temperature here (see Figure 2.15) over the relevant region of the atmosphere. This is in spite of their using a mean molecular weight $\mu=2.3$, which assumes molecular hydrogen, while here the mean molecular weight in the atomic layer is closer to $\mu \simeq 1.3$, smaller by a factor of 2 . Such a large difference in temperature cannot be explained by the abundance variations due to ionization seen in Figure 2.5.

One indication that higher temperatures than found in Huitson et al. (2012) and Wyttenbach et al. (2015) are required comes from the inferred range of density between line center and line wing. A lower temperature means a larger density difference between the base of the atmosphere, where the continuum forms, and higher altitudes where the line center forms. An underestimate of this density decrease can be found by using the highest fitted temperature used in Wyttenbach et al. (2015), $T=3270 \mathrm{~K}$, and mean molecular weight $\mu=2.3$. Assuming the pressure is large, 1 bar at the continuum altitude, also errs on the side of high density higher in the atmosphere. With 


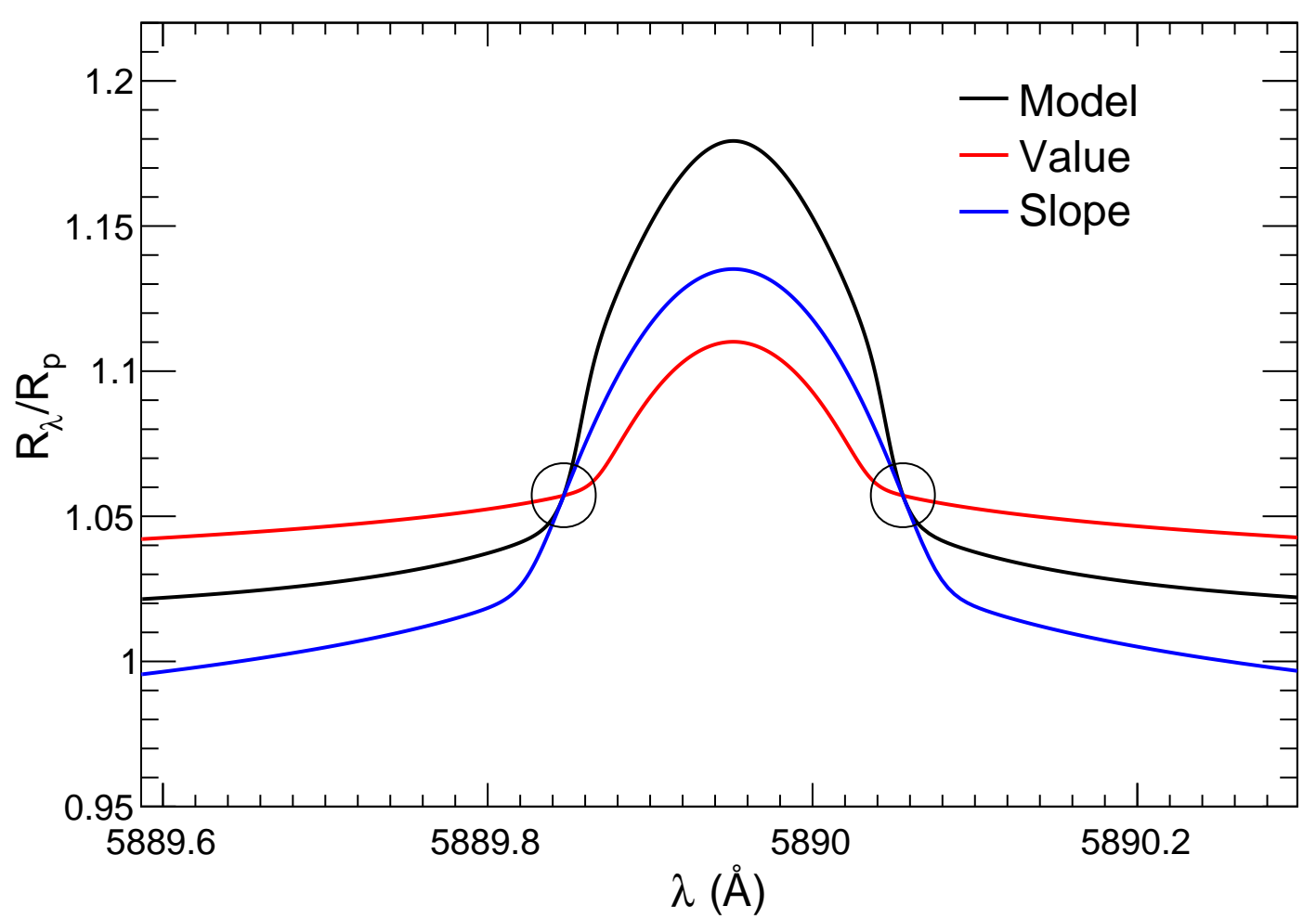

Fig. 2.14. - Comparison of the fiducial model transmission spectrum and two different fits at a single point (circled) using the isothermal transit radius approximation in Equation 2.40. The black line shows the transit radius of the Na I 5890 line for the fiducial " $\xi=1, P_{\mathrm{b}}=10 \mu$ bar" model (see Figures 2.12 and 2.13). The red curve is chosen to agree with the value of the transit radius at $r(\lambda)=1.057 R_{p}$, and with the continuum $r(\lambda)$ on the line wing. This requires a temperature $T=2830 \mathrm{~K}$, assuming $\mu=2.3$. The blue curve is chosen to have the same slope as the black curve at the circled point, which requires $T=6010 \mathrm{~K}$ for $\mu=2.3$.

these two assumptions, an isothermal atmosphere gives the pressure $5 \times 10^{-5} \mu \mathrm{bar}$ at the line center altitude $1.27 \times 10^{4} \mathrm{~km}$. To be optically thick to the Na I 5890 line, the ground state density must be $n_{\mathrm{Na}_{\mathrm{I}}} \gtrsim\left(\sigma_{\mathrm{NaD} 2} \sqrt{2 \pi r H}\right)^{-1} \simeq 10^{2} \mathrm{~cm}^{-3}$, where $\sigma_{\mathrm{NaD} 2}$ is the line center cross section of $\mathrm{Na}$ I 5890. This requires a $\sim 10^{-6}$ mixing ratio of $\mathrm{Na}$ I, which means $\mathrm{Na}$ has to be mostly neutral at this altitude if the atmosphere is in solar abundance. However, because the atmosphere is optically thin to the stel- 


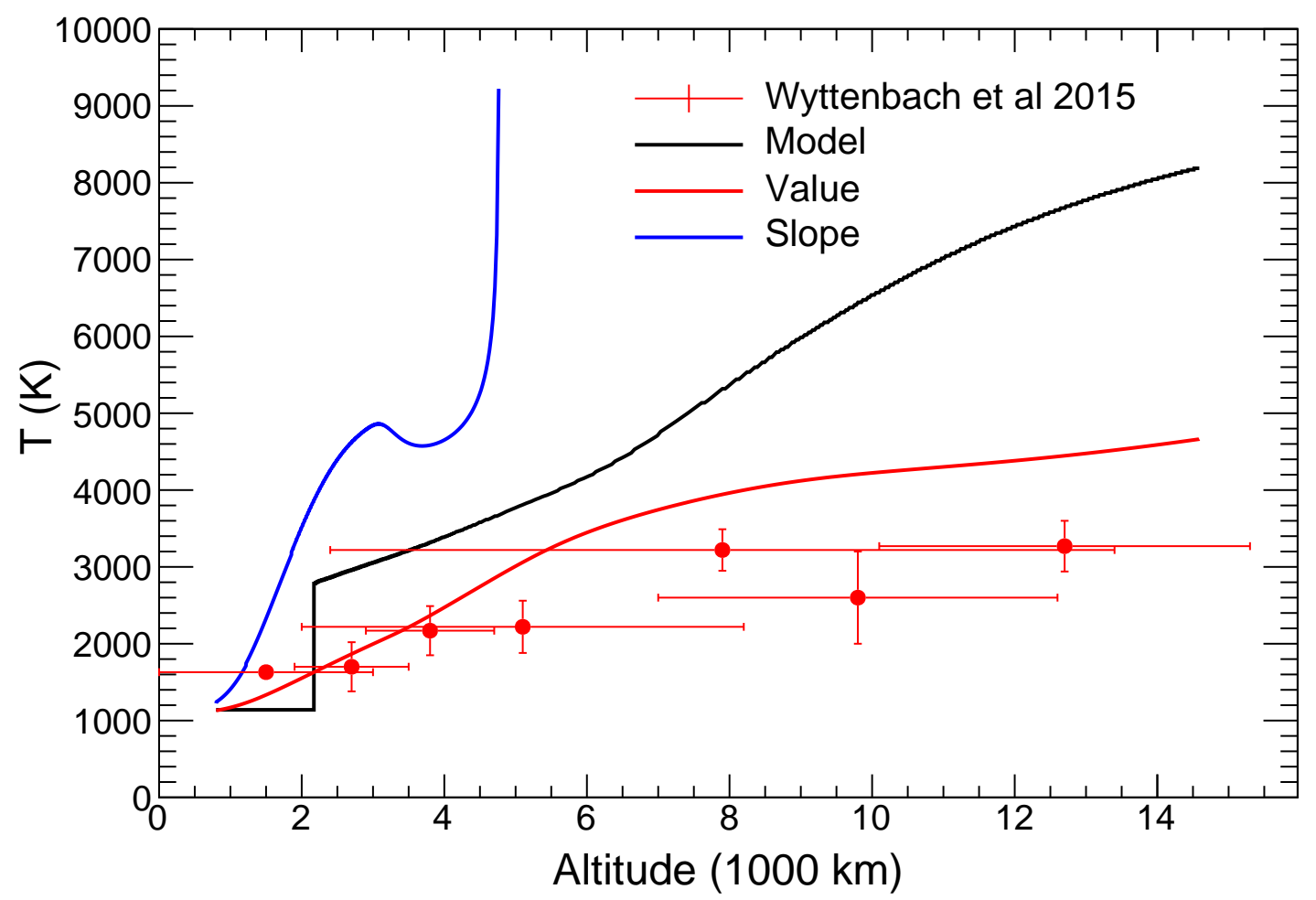

Fig. 2.15. - Retrieved temperature profile using two different fitting strategies shown in Figure 2.14. Temperature profile fitted from the observed NaD transmission spectrum obtained by Wyttenbach et al. (2015) is shown with red circles.

lar flux at this pressure, $\mathrm{Na}$ is significantly ionized (see the left hand side of Figure 2.5). Therefore, the highest temperature measured in Wyttenbach et al. (2015) may underestimate the line center temperature formed high in the atmosphere.

For sufficiently high spectral resolution data, $r(\lambda)$, with high signal to noise for each data point, and for an atmosphere which is nearly isothermal and with small abundance gradients, the temperature of the atmosphere will be accurately recovered using Equation 2.40 in the plane-parallel limit. However, in an atmosphere where temperature increases upward rapidly, this method tends to underestimate the temperature (Wyttenbach et al. 2015). The problem is exacerbated when the opacity is 
provided by the line's Doppler core at the altitudes of interest.

To better understand the retrieval of a temperature profile for the non-isothermal, non-constant abundance case, an example is given here to fit the fiducial model $r(\lambda)$ (see Figures 2.12 and 2.13) with the isothermal profile in Equation 2.40. This eliminates measurement errors in the data, and a fine enough grid of points is used so that numerical error is negligible. The black curve in Figure 2.14 shows the fiducial model for the Na I 5890 absorption profile, the same as in Figure 2.13. Two methods are used to fit Equation 2.40 to the fiducial model. "Method 1" is equivalent to that in Wyttenbach et al. (2015). The constant term in Equation 2.40 is chosen in order that the absorption depth is 0 at $5912 \AA$, on the line wing. The temperature $T(r)$ at each radius $r(\lambda)$ is determined by matching the value of the transit radius using Equation 2.40 to the fiducial model. A mean molecular weight $\mu=2.3$ is used, as in Wyttenbach et al. (2015). Figure 2.14 shows an example of a Method 1 fit with $T=2830 \mathrm{~K}$, which matches the value of the absorption depth at $R=1.057 R_{p}$. When the value is fitted, the slope will be smaller than that of the fiducial model. "Method 2" is equivalent to that in Huitson et al. (2012). By adjusting $H$ and the constant term at each $r(\lambda)$, Equation 2.40 is used to match the slope of the fiducial model transit radius. Again $\mu=2.3$ is used. Figure 2.14 shows an example of a Method 2 fit with $T=6010$ that is tangent to the fiducial model $r(\lambda)$ curve at $R=1.057 R_{p}$.

The retrieved temperature profiles for Method 1 and Method 2 are compared to the fiducial model temperature profile in Figure 2.15. For comparison, the Method 2 temperature profile estimated from the data by Wyttenbach et al. (2015) is shown as the points with error bars. Given that there is no numerical noise in this example, as the isothermal $r(\lambda)$ is fit to a theoretical model, the disagreement between the Method 1 and Method 2 fits and the true temperature profile is quite large. The 
disagreement would be even larger if the more appropriate $\mu \simeq 1.3$ was used near line center. Method 1 can reasonably retrieve the temperature in the molecular layer, where the fiducial model temperature is constant and $\mu=2.3$. However, the retrieved temperature in the atomic layer is lower than the fiducial model where the temperature increases outward. Although the retrieved temperature is still higher than the points from Wyttenbach et al. (2015), this example partially explains the lower inferred temperature in that work as compared to the fiducial model in this work.

In the Doppler core, Equation 2.40 gives

$$
r(\lambda)-r\left(\lambda_{0}\right)=-H\left(\frac{\Delta \nu}{\Delta \nu_{D}}\right)^{2}=-6000 \mathrm{~km}\left(\frac{\Delta \lambda}{0.1 \AA}\right)^{2},
$$

independent of temperature. By contrast, the fiducial model $r(\lambda)$ is steeper than Equation 2.41 because the absorption by a higher and hotter atmosphere layer produces a broader absorption than if the temperature is constant. This effect leads to an overestimate in temperature at the base of the atomic layer. Because of the combination of these two effects, the slope of a section of the fiducial model is too steep and Equation 2.40 cannot produce such a steep slope for any temperature. Near the line core, the slope of $r(\lambda)$ for the fiducial model also depends on the Na I abundance gradient. Collisional ionization by thermal electrons and Ly $\alpha$ photoionization decrease the $\mathrm{Na}$ ionization fraction at level above $0.1 \mu$ bar (see Figure 2.5), which can decrease the slope near the line center.

These results suggest that the isothermal model may not accurately retrieve a rapidly rising temperature profile. In comparison, fitting the whole wavelength range with an atmosphere model contains several isothermal layers, or a single layer with a continuous temperature profile may better constrain the atmosphere temperature. 


\subsubsection{Impact of LyC Flux on Transit Depth}

HD 189733 is known to be an active star (Boisse et al. 2009; Pillitteri et al. 2014, 2015). To show the dependence of the transmission spectrum on the stellar EUV/Xray flux, two more models with an extra LyC flux multiplier factor $\xi=1 / 4$ and $\xi=4$ are applied. Figure 2.9 shows that a stronger LyC flux will make the $\mathrm{H} \alpha$ transit depth deeper. This is consistent with the conclusion in Cauley et al. (2017) that the $\mathrm{H} \alpha$ transit showing the largest absorption value occurs when the star is the most active. In comparison, Figure 2.13 shows that LyC flux has no effect on the $\mathrm{NaD}$ transmission spectrum. The fact that the maximum transit depth suggested by Wyttenbach et al. (2015) and Cauley et al. (2016) for NaD are similar when binned to the same $0.2 \AA$ resolution agrees with Figure 2.13.

The reason of this difference is that the NaD transmission spectrum only depends on the temperature or scale height of the atmosphere below $10^{-2} \mu$ bar, which is mostly heated by photoelectric heating of metals by photons with energy less than $13.6 \mathrm{eV}$ and not sensitive to the LyC flux. In contrast, $\mathrm{H} \alpha$ not only depends on the atmosphere temperature, but also on the $\operatorname{Ly} \alpha$ intensity in this region, which is larger for a strong LyC flux environment. The $\mathrm{H} \alpha$ transmission spectrum also becomes slightly broader in the strong LyC flux case, because the $\mathrm{H} \alpha$ becomes optically thick at higher and hotter part of the atmosphere in this case. Comparing the variability of the transit depths of the $\mathrm{H} \alpha$ and $\mathrm{NaD}$ lines is a possible method to break the degeneracy between the transit depth variability due to blocking an active region on the star surface and the change in the atmosphere due to stellar activity. 


\subsubsection{Impact of Metallicity on Transit Depth}

The metallicity is crucial in the model presented in this paper, but its value is uncertain. Since $\mathrm{Mg}$ is the dominant coolant in the model, an atmosphere with no $\mathrm{Mg}$ is calculated to assess the effect of metallicity on the transmission spectrum. Because the atmosphere is warmer and more extended without $\mathrm{Mg}$ cooling, a deeper $\mathrm{H} \alpha$ and NaD transit depth result, as shown in Figures 2.9 and 2.13. The relatively large transit depth difference between two models with and without $\mathrm{Mg}$ indicates that a high precision $\mathrm{H} \alpha$ and $\mathrm{NaD}$ transmission spectrum measurement can constrain the metallicity in the upper atmosphere.

\subsection{Discussion}

\subsubsection{Other Possible Cooling Mechanisms}

Adiabatic cooling is another potential cooling mechanism discussed in the literature. Koskinen et al. (2013a) constructed an atmosphere model for a similar hot Jupiter HD 209458b. In their model, the stellar heating is mainly balanced by adiabatic cooling. Compared with HD 189733b system, the LyC flux of HD 209458 is weaker and the orbit of HD 209458b is further away from the star. They also introduced a factor of 1/4 reduction on stellar flux to account for uniform day-night heat redistribution. As a result of these differences, the heating rate of Koskinen et al. (2013a) HD 209458b atmosphere model is more than 20 times smaller than the rate in our HD 189733b model. On the other hand, the adiabatic cooling does not differ much in two systems because of the similar mass loss rate (Murray-Clay et al. 2009). Therefore, the adiabatic cooling is unlikely to be the answer in the case of HD 189733b.

The effect of the adiabatic cooling of the model here can be estimated with an 
assumed mass loss rate. Including the adiabatic cooling in the pressure coordinate system according to Bildsten (1998), the entropy equation takes the following form,

$$
\sum H-\sum C=-C_{p} \frac{\dot{M} \rho g_{p}}{4 \pi R_{p}^{2}} \frac{\mathrm{d} T}{\mathrm{~d} P}+\frac{C_{p} \dot{M} T \rho g_{p}}{4 \pi R_{p}^{2} P} \nabla_{\mathrm{ad}}
$$

where $\sum H$ and $\sum C$ stand for the sum of heating and cooling rates respectively as shown in the right and left hand side of Equation $2.24, \nabla_{\mathrm{ad}}=(\mathrm{d} \ln T / \mathrm{d} \ln P)_{S}$ is the adiabatic temperature gradient at constant entropy, $\dot{M}$ is the mass lost rate, and $C_{p}$ is the specific heat per unit mass at constant pressure. In the fiducial model, the temperature gradient is about $\mathrm{d} T / \mathrm{d} r=7 \times 10^{-7} \mathrm{~K} \mathrm{~cm}^{-1}$ at the radius that $13.6 \mathrm{eV}$ photon becomes optically thick, where most LyC photons get absorbed. Applying the mass-loss rate $\dot{M}=4 \times 10^{9} \mathrm{~g} \mathrm{~s}^{-1}$ suggested by Salz et al. (2016) using a hydrodynamic escaping atmosphere model, the adiabatic cooling rate is $1.1 \times 10^{-8} \mathrm{erg} \mathrm{cm}^{-3} \mathrm{~s}^{-1}$ and the second term in the Equation 2.42 is the dominant source. Comparing to the heating and cooling rates shown in Figure 2.4, the adiabatic cooling is more than two orders of magnitude smaller in the region where $\mathrm{H} \alpha$ mostly absorbed, and may only become important in the region above $P \sim 3 \times 10^{-4} \mu$ bar. The mass-loss rate may be model dependent. An upper bound for the mass loss rate can be found using the energy-limited escape rate (Murray-Clay et al. 2009), which assumes all LyC flux converts to unbinding the atmosphere. The LyC flux $F_{\mathrm{LyC}}=1.4 \times 10^{4} \mathrm{erg} \mathrm{cm}^{-2} \mathrm{~s}^{-1}$ corresponds to the energy-limited mass-loss rate $\dot{M}=1.6 \times 10^{11} \mathrm{~g} \mathrm{~s}^{-1}$. In this case, the adiabatic cooling rate is $4 \times 10^{-7} \mathrm{erg} \mathrm{cm}^{-3} \mathrm{~s}^{-1}$ and still has a less than $10 \%$ effect in the region mainly concerned. 


\subsubsection{Comparing with Other Hot Jupiter Upper Atmosphere Models}

Christie et al. (2013) The present study agrees with the conclusion in Christie et al. (2013) that the $2 p$ occupation is set by radiative excitation and de-excitation, and as an improvement, we include a Ly $\alpha$ radiation transfer inside the atmosphere instead of applying a constant solar Ly $\alpha$ intensity. Because of the $\sim 30$ times stronger stellar Ly $\alpha$ intensity of HD 189733 comparing to the Sun and considering the Ly $\alpha$ photons generated inside the atmosphere due to collisional excitation and recombination cascades, the Ly $\alpha$ mean intensity should be $\sim 100$ times larger for the majority of the atomic layer. In addition, because Christie et al. (2013) underestimates the $n_{2 p}$ by a factor of 20 due to a math error, the $n_{2 p}$ should be significantly larger in the whole simulation domain, and thus the atmosphere is optically thick to $\mathrm{H} \alpha$ mainly due to the absorption of $\mathrm{H}(2 p)$. The observed $\mathrm{H} \alpha$ absorption width agrees well with an optically thick atmosphere model.

Because of the much larger $n_{2 \ell}$, the photoionization of $\mathrm{H}(2 \ell)$ is larger compared to photoionization of the ground state in the atomic layer. Hence the $n_{e}$ and $n_{p}$ in this work is $\sim 10$ times larger.

Considering the proton collisional $\ell$-mixing process with rate $\sim 10$ times larger than electron collisional process, as well as the large $\mathrm{H}(2 \mathrm{p})$ population, the creation of $2 \mathrm{~s}$ hydrogen is dominated by $\ell$-mixing rather than collisional excitation considered in Christie et al. (2013). As a result, $2 s$ and $2 p$ reach collisional equilibrium.

In addition, it is shown that the metal lines are crucial in cooling the atmosphere. Assuming solar abundance, lines of $\mathrm{Mg}$ and $\mathrm{Na}$ can cool the atomic layer by $\simeq 2000-$ $3000 \mathrm{~K}$. 
Menager et al. (2013) Menager et al. (2013) investigate the Ly $\alpha$ emission and reflection by the atmosphere of HD 189733b. The temperature and electron, hydrogen, and helium number density profiles of HD 189733b from the Koskinen et al. (2011) unpublished model were applied. The temperature, $n_{e}, n_{p}$, and $n_{\mathrm{H}}$ are in broad agreement with the profiles presented in this paper in the corresponding pressure range. According to a similar model of HD 209458b presented in Koskinen et al. (2013a), it should be a one-dimensional hydrodynamic model of the upper atmosphere considering hydrogen and helium constructed on top of a full photochemical model of the lower atmosphere. They chose the average solar flux as their stellar spectrum, which is $\sim 10$ times smaller than the synthetic spectrum from X-exoplanets used in the present model in EUV/X-ray. Different from the photoelectron heating efficiency $\eta(E)$ calculated at the fixed ionization fraction $x_{e}=0.1$ throughout the model, or a constant $\eta$ applied in the Koskinen et al. (2013a), a $\eta$ based on the local $x_{e}$ is used in this work.

The temperature in the HD 189733b model of Koskinen et al. (2011) reachs a peak of about $13000 \mathrm{~K}$ at a pressure of $3 \times 10^{-4} \mu \mathrm{bar}$. The adiabatic cooling lowers the temperature at higher altitude. Their temperature at pressure range $10^{-3}$ to 1 $\mu$ bar is higher by about $3000 \mathrm{~K}$. Two possible reasons of this difference are Koskinen et al. (2013a) do not consider metal lines cooling, which are the dominant cooling mechanisms in our model, and conduction is not included in this work, which is a net heating in this pressure range according to their result. Their temperature decreases much faster with pressure above $1 \mu$ bar comparing to Figure 2.3. The lack of molecular cooling in this work is the possible reason. Their $n_{e}$ decreases slowly with pressure in the atomic layer and is $\sim 10$ times smaller at $10 \mu$ bar compared to this work. This difference is the result of missing the photoionization from $\mathrm{H}(2 s)$, 
which is the dominant ionization mechanism in this region.

In the Ly $\alpha$ radiation transfer simulation of Menager et al. (2013), the Ly $\alpha$ photons emitted by the star and by the planetary atmosphere are considered. However, when considering the Ly $\alpha$ photon emitted by the planetary atmosphere, the Ly $\alpha$ photon created through recombination cascades is not included. In order to calculate the Ly $\alpha$ thermal excitation in the atmosphere, the number densities of $2 s$ and $2 p$ states hydrogen are modeled with a level population study. In the $2 p$ state level equation, Ly $\alpha$ excitation, which completely dominates the $2 p$ state, is not included. In addition, because the $p$ collisional $\ell$-mixing process is missing in their model, the $2 \ell$ state number densities result shown are affected.

Menager et al. (2013) claimed that the thermal emission of HD 189733b contributes to $6 \%$ of the total intensity of the Ly $\alpha$ line. In the fiducial model of this work, this ratio is $3.7 \times 10^{3} \mathrm{erg} \mathrm{cm}^{-2} \mathrm{~s}^{-1} /\left(2.0 \times 10^{4} \mathrm{erg} \mathrm{cm}^{-2} \mathrm{~s}^{-1}+3.7 \times 10^{3} \mathrm{erg} \mathrm{cm}^{-2} \mathrm{~s}^{-1}\right)=$ $16 \%$. This ratio strongly depends on the metallicity as well as the stellar LyC to Ly $\alpha$ flux ratio.

\subsection{Conclusion}

A detailed one-dimensional hydrostatic atmosphere model is constructed over the region dominated by atomic hydrogen and comparison of model transmission spectra to the data has been made. An atomic hydrogen level population calculation and a Monte-Carlo Ly $\alpha$ radiation transfer are done to model the abundance of $2 \ell$ state hydrogen. The model transmission spectra are in broad agreement with the HD 189733b data for both the line center absorption depth and the line width, although the comparison is complicated by the observed variability.

The Ly $\alpha$ radiation transfer shows that the Ly $\alpha$ has a very broad line width with 
a flat top due to the resonant scattering process. The line profile weighted mean intensity $\bar{J}_{L y \alpha}$ is large and roughly constant down to the $P=0.1 \mu$ bar level of the atmosphere. Ly $\alpha$ photons created inside the atmosphere and incident from the star are both important. The Ly $\alpha$ source function extends deep into the atmosphere due to ionization from progressively higher energy stellar LyC photons. The stellar Ly $\alpha$ photon can penetrate into very large line center optical depth because the stellar Ly $\alpha$ intensity is much boarder than the Doppler width inside the atmosphere. The stellar Ly $\alpha$ photons incident through the surface and the photons generated above $10^{-2} \mu \mathrm{bar}$ can mostly escape through the top boundary. In contrast, the photons emitted below $0.1 \mu$ bar are mostly absorbed during the resonant scattering processes due to the high optical depth. For $P \gtrsim 0.1 \mu$ bar, $\bar{J}_{L y \alpha} \propto P^{-1}$.

The $n_{2 p}$ is determined by the radiative rates between $1 s$ and $2 p$ throughout the simulation domain because of the large Ly $\alpha$ intensity. The $2 s$ and $2 p$ states reach collisional equilibrium by the large $p$ collisional $\ell$-mixing rate, which was overlooked in this context. The combination of the decreasing Ly $\alpha$ excitation rates and the increasing hydrogen density gives rise to a nearly flat $n_{2 \ell}$ over two decades in pressure. This layer is optically thick to $\mathrm{H} \alpha$, and the temperature is in the range $T \simeq 3000-$ 8500 K. Both $\mathrm{H} \alpha$ and $\mathrm{NaD}$ are optically thick up to the altitude with $P \sim 10^{-2} \mu$ bar, which corresponds to the atomic layer of the atmosphere. Assuming solar abundance, radiative cooling due to metal species dominates over the entire model, with $\mathrm{Mg}$ and $\mathrm{Na}$ being the two most important species.

Additional models computed for a range of the stellar LyC flux find transit depth of $\mathrm{H} \alpha$ changes with LyC level, suggesting that the variability in $\mathrm{H} \alpha$ transit depth may be due to variability in the stellar LyC. In contrast, the $\mathrm{Na}$ absorption profile is insensitive to the LyC level. Since metal lines provide the dominant cooling of this 
part of the atmosphere, the atmosphere structure is sensitive to the density of species such as Mg and Na, which may themselves be constrained by observations. Lastly, since the $\mathrm{H} \alpha$ and $\mathrm{NaD}$ lines have comparable absorption depths for the same spectral resolution, we argue that the center of the $\mathrm{NaD}$ lines are also formed in the atomic layer where the $\mathrm{H} \alpha$ line is formed.

The present model is in agreement with the observed NaD transmission spectrum by Huitson et al. (2012) and Wyttenbach et al. (2015), although the inferred atmospheric temperature is significantly larger than that found assuming an isothermal profile and molecular composition. It is shown that the temperature achieved by fitting each wavelength interval in the observed transmission spectrum with an isothermal atmosphere model may not accurately retrieve the original temperature profile, if the temperature increases rapidly with the altitude. 


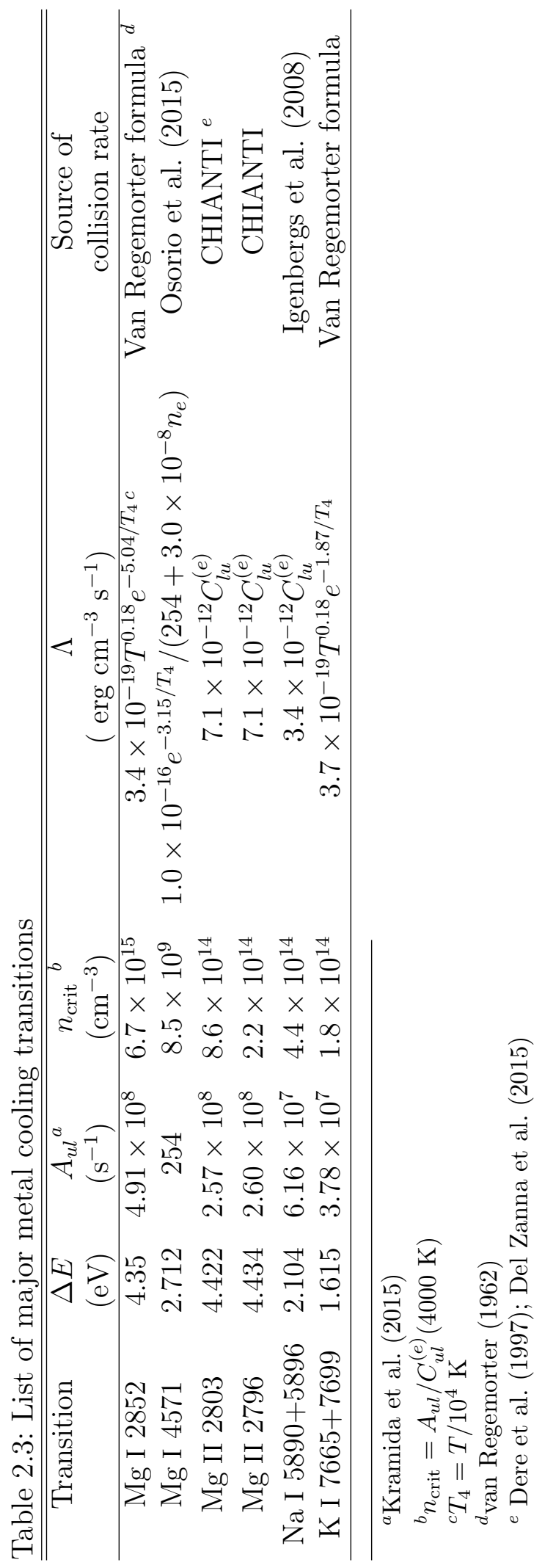




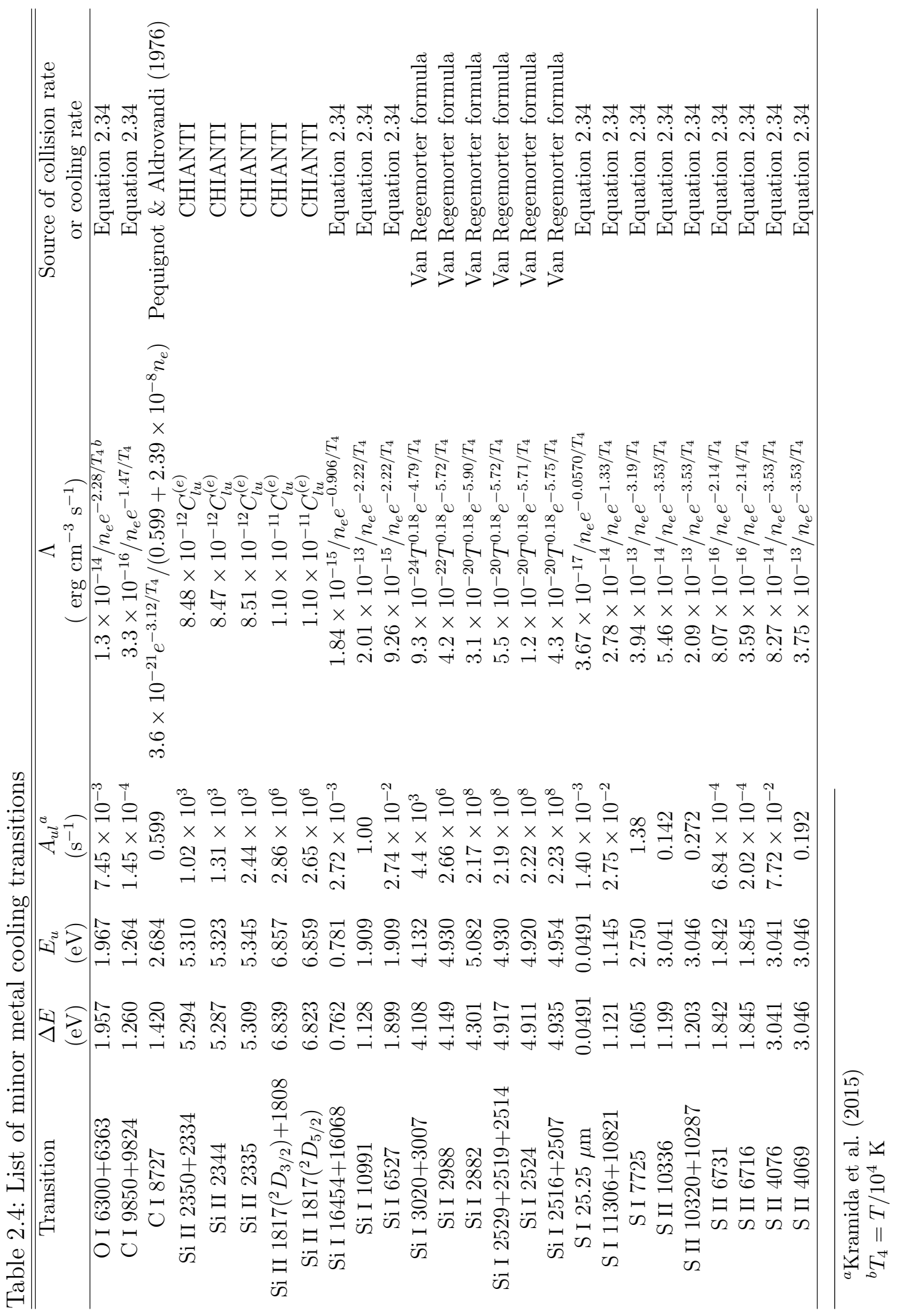




\section{Chapter 3}

\section{Electron Scattering Wings on Lines in Interacting Supernovae}

This chapter is based on the paper draft, Huang \& Chevalier (In preperation).

\subsection{Introduction}

The effects of electron scattering on emission lines have been discussed in various contexts. One is an explanation for the broad emission lines observed in Seyfert galaxies (Weymann 1970; Laor 2006), although this is not currently the preferred explanation for broad lines. In an expanding medium, electron scattering is expected to produce to produce a wing on the red side of an emission line. Auer \& van Blerkom (1972) noted the possible relevance of this process to Wolf-Rayet stars and Seyfert galaxies (see also Hillier 1991). In the context of supernovae, Fransson \& Chevalier (1989) examined the effect of electron scattering on lines formed in the

freely expanding ejecta during the nebular phase. As above, scattering in the radially expanding gas gives a red wing to the line. In the case where the thermal velocities 
of electrons dominate, the scattering primarily has a symmetric broadening effect about zero velocity. This is the case studied by Chugai (2001) for application to early spectra of the Type IIn supernova SN 1998S. In this scenario, after the supernova shock wave has broken out of the progenitor star and ionizing radiation from the shock region is able to ionize the surroundings, the circumstellar medium around the supernova shock has substantial optical depth to electron scattering. This situation can occur in a supernova with a dense circumstellar medium because a viscous shock is expected to form when the optical depth to the shock is $\sim c / v_{s}$, where $c$ is the speed of light and $v_{s}$ is the shock velocity (e.g., Chevalier \& Irwin 2011; Katz et al. 2011)

The observational signature of electron scattering is broad wings (1000's of km $\mathrm{s}^{-1}$ ) on a narrow line feature with velocities that are characteristic of the circumstellar medium. Electron scattering line profiles have been calculated and applied to a number of observed supernovae, including SN 1998S (Chugai 2001), SN 2005gj (Aldering et al. 2006), SN 2011ht (Humphreys et al. 2012), and SN 2010jl (Fransson et al. 2014; Borish et al. 2015; Dessart et al. 2015). In addition, electron scattering has been mentioned as probably important for other supernovae, such as SN 2008am (Chatzopoulos et al. 2011). Supernovae with narrow spectral lines that have electron scattering wings are naturally classified as Type IIn (narrow line). However, electron scattering is probably not a factor in all Type IIn supernovae because they have a range of circumstellar densities, and at late times the electron scattering optical depth is expected to become small as the supernova shock wave sweeps up the scattering circumstellar gas. The mass motions then become the dominant factor in the line profiles.

Our primary aim here is to treat the line wings outside of the unscattered line 
emission. We assume that the gas is optically thin in the unscattered line, as did Auer \& van Blerkom (1972) and Chugai (2001), recognizing that there may be significant optical depth in the line, especially for $\mathrm{H} \alpha$. Calculations including these effects were carried out for Wolf-Rayet stars (Hillier 1991), SN 1994W (Dessart et al. 2009, 2016) and SN 2010jl (Dessart et al. 2015). This calculation requires the treatment of the non-equilibrium level populations in the radiation field of the object. There is considerable uncertainty in these quantities for supernovae. The physics of the scattered line wings is relatively straightforward and is the case studied here. The aim is to find diagnostics provided by observations of the broad line component that are relatively model independent.

Although electron scattering has frequently been invoked for broad lines in interacting supernovae, there has not been a systematic investigation of the line properties. We investigate here the dependence of the line profiles on various parameters, including the optical depth, the density distribution of the circumstellar gas, and the velocity profile of the circumstellar gas. These properties provide potential diagnostics of the supernova interaction. The basic theory and results are presented in Section 3.2.2. Applications to observed supernovae are in Section 3.2.3. The results are discussed in Section 3.4.

\subsection{Scattering in a Circumstellar Medium}

As in many previous treatments of electron scattering, we used a Monte Carlo scheme to calculate the effects of scattering. Because our primary application is to the optical spectra of Type IIn supernovae, we assume scattering in the Thomson limit, using the Thomson scattering differential cross section relation for each photon scattering. The effect of polarization is ignored; our models are spherically symmetric. To make 
the simulation more efficient, statistical weights were assigned to the photons as described in section 9.3 of Pozdnyakov et al. (1983). The simulations were carried out in spherical symmetry, with the scattering medium between an inner radius $R_{1}$ and an outer radius $R_{2}$ (Fig. 3.1). The optical depth $\tau$ through the medium is along the radial direction. A test of the code was provided by the analytical solution of Weymann (1970); we found good agreement of the Monte Carlo calculations with this solution.

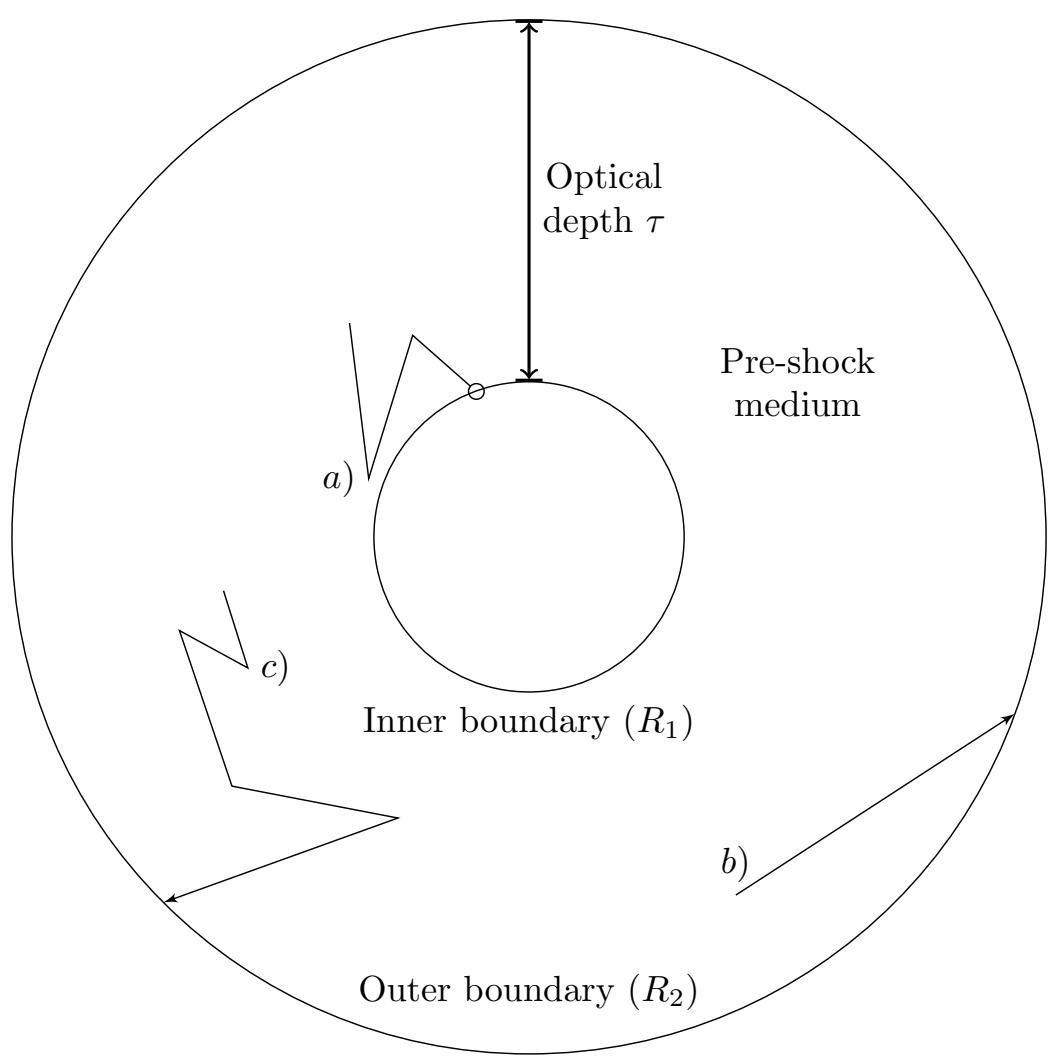

Fig. 3.1. - Illustration of the scattering region, which extends from $R_{1}$ (inner boundary) to $R_{2}$ (outer). A photon generated in the wind gas can a) be absorbed by the inner boundary, $b$ ) escape the medium from the outer boundary without any scattering, contributing to the narrow component of the spectrum, and $c$ ) escape from the outer boundary after scattering a certain number of times, contributing to the broad component. 


\subsubsection{The Single Scattering Limit}

As an initial case, we calculated the situation where there is only single scattering by a thermal distribution of electrons. This case depends only on the broadening by thermal electrons and does not depend on the parameters for the circumstellar gas other than the temperature. In a realistic situation, the broad line profile should approach this case in the low optical depth limit. Fig. 3.2 shows the resulting distribution of scattered photons for an assumed electron gas temperature of 20,000 K. It can be seen that the profile over the first factor $\sim 4$ in flux is approximately exponential and steepens from an exponential beyond that. The full width at half maximum (FWHM) of the line can be expressed as $\Delta v=913(T / 20,000 \mathrm{~K})^{1 / 2} \mathrm{~km} \mathrm{~s}^{-1}$. To obtain a robust measurement of the FWHM, we fit an exponential to a $\sim 100 \mathrm{~km} \mathrm{~s}^{-1}$ region near the profile peak and at the half maximum bin. This method helped to account for fluctuations in the Monte Carlo results. For comparison, the mean thermal velocity of an electron at $20,000 \mathrm{~K}$ is $954 \mathrm{~km} \mathrm{~s}^{-1}$. The line profile compares well with the single scattering result shown in Fig. 2 of Sunyaev (1980), whose result has some asymmetry because $5.1 \mathrm{keV}$ photons are considered and the situation is not fully in the Thomson limit. In the following discussion, we bin non-scattered and scattered photons (narrow and broad component) separately in order to measure a well defined FWHM of the broad component.

\subsubsection{Stationary Circumstellar Medium}

Our aim is to present physically plausible situations where electron scattering plays a role. We begin by treating simpler situations and proceed to more complex ones. The initial case is an ionized wind with density profile $\rho_{w} \propto r^{-2}$, as expected for a steady wind. The wind velocity is assumed to be negligible so the circumstellar gas is 


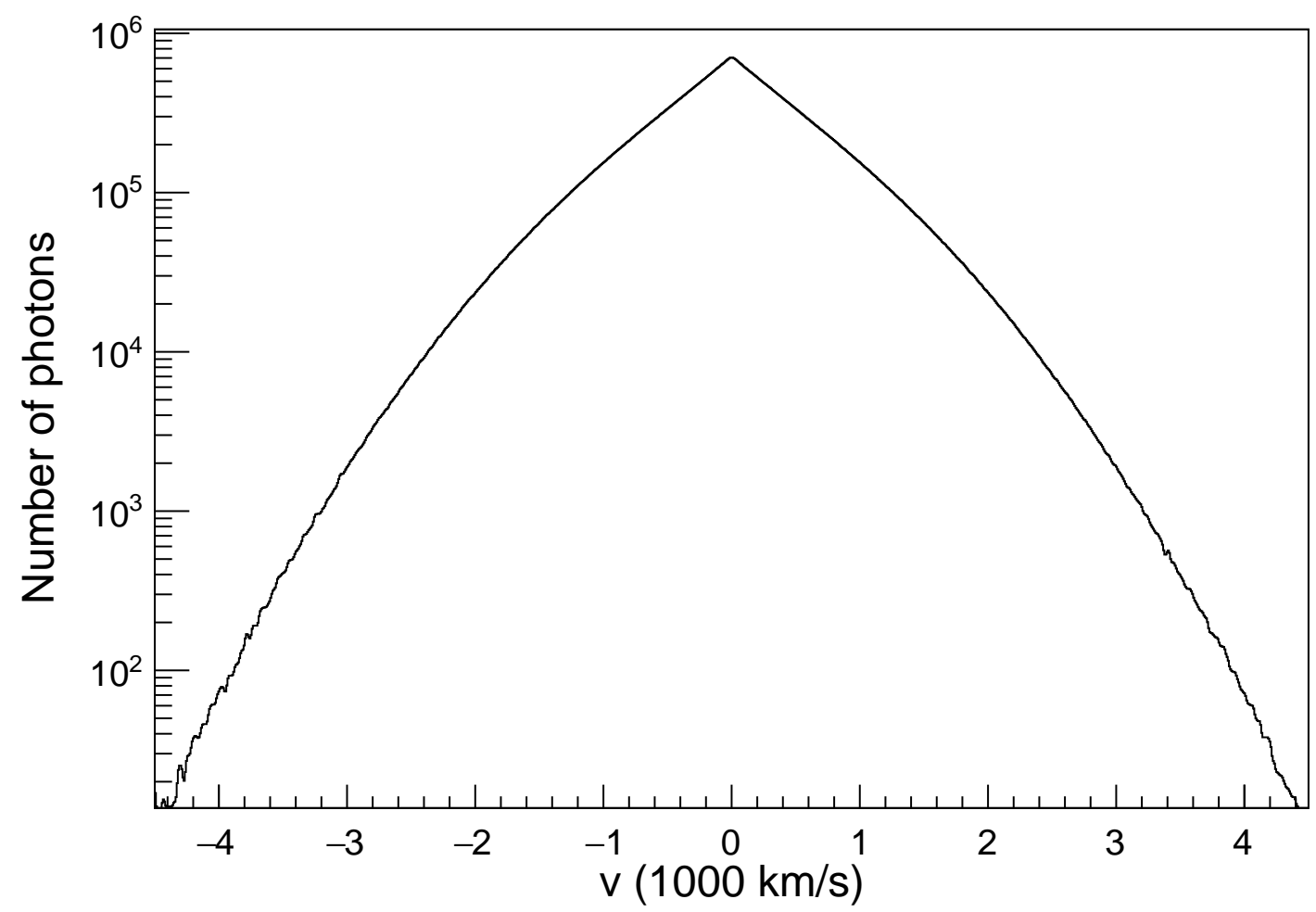

Fig. 3.2. - Line profile for single scattering by thermal electrons with $T=20,000 \mathrm{~K}$.

effectively stationary. The inner boundary is assumed to be an absorbing sphere; this might be a shocked shell that is not radiating. Most of the contribution to the optical depth and emissivity comes from close to the inner boundary for this density profile. We assume that the wind gas is responsible for both the emission of line photons and the subsequent scattering of these photons. The line emissivity is taken to be $\propto n^{2}$, where $n$ is the gas density. Under these conditions, the broad component of the line profile depends only on the optical depth to the absorbing sphere, $\tau$, and the radial extent of the scattering electrons.

Fig. 3.3 shows the resulting line profiles for different values of $\tau$. The ratio $R_{2} / R_{1}$ is $10^{3}$. As expected, higher optical depths lead to multiple scattering and a broader broad component than is obtained in the single scattering case. There is also a 
narrow component that is made up of line photons that escape without scattering. To characterize the width of the approximately exponential scattering wing, the full width at half maximum (FWHM) of the broad (scattered) component is used here. Fig. 3.4 shows the normalized line shapes such that the line profiles have the same FWHM. It can be seen that the line profile shapes are similar over the top part of the profile, but that the line wings are relatively stronger at high $\tau$. At low $\tau$, the line profile drops more rapidly than an exponential out in the wings, while at high $\tau$, the profile drops less rapidly than an exponential. At $\tau \approx 10$, the profile remains exponential far out in the wings. The results are shown for a particular temperature $T$, but the electron thermal velocity is $\propto T^{1 / 2}$, so that the FWHM $\propto T^{1 / 2}$. The FWHM of the broad component is shown in Fig. 3.5 as a function of $\tau$. At low optical depth, the FWHM approaches the single scattering value.

One parameter is the radial extent of scattering electrons. Fig. 3.6 shows the line profiles resulting from a number of different extents. The profile is expected to depend only on the ratio $\eta=R_{2} / R_{1}$. It can be seen that, provided that $R_{2} / R_{1} \gtrsim 20$, the line profile depends weakly on the radius ratio. This conclusion also holds for other power law density profiles except a negative power law index $\sim-1$ (see below for more discussion). Fig. 3.6 also shows that the line formed over a narrow radial region, which approximates a planar scattering layer, gives a broader line than in the case of a large radial extent. The result can be understood in that a photon escaping from the vicinity of a spherical region inner boundary sees a similar optical depth in various directions while, in the planar case, the optical depth seen by photons is generally higher than that along a line perpendicular to the planar surface. The planar case thus leads to a higher mean optical depth and broader lines.

Fig. 3.3 shows the presence of the narrow component mentioned above. The ratio 


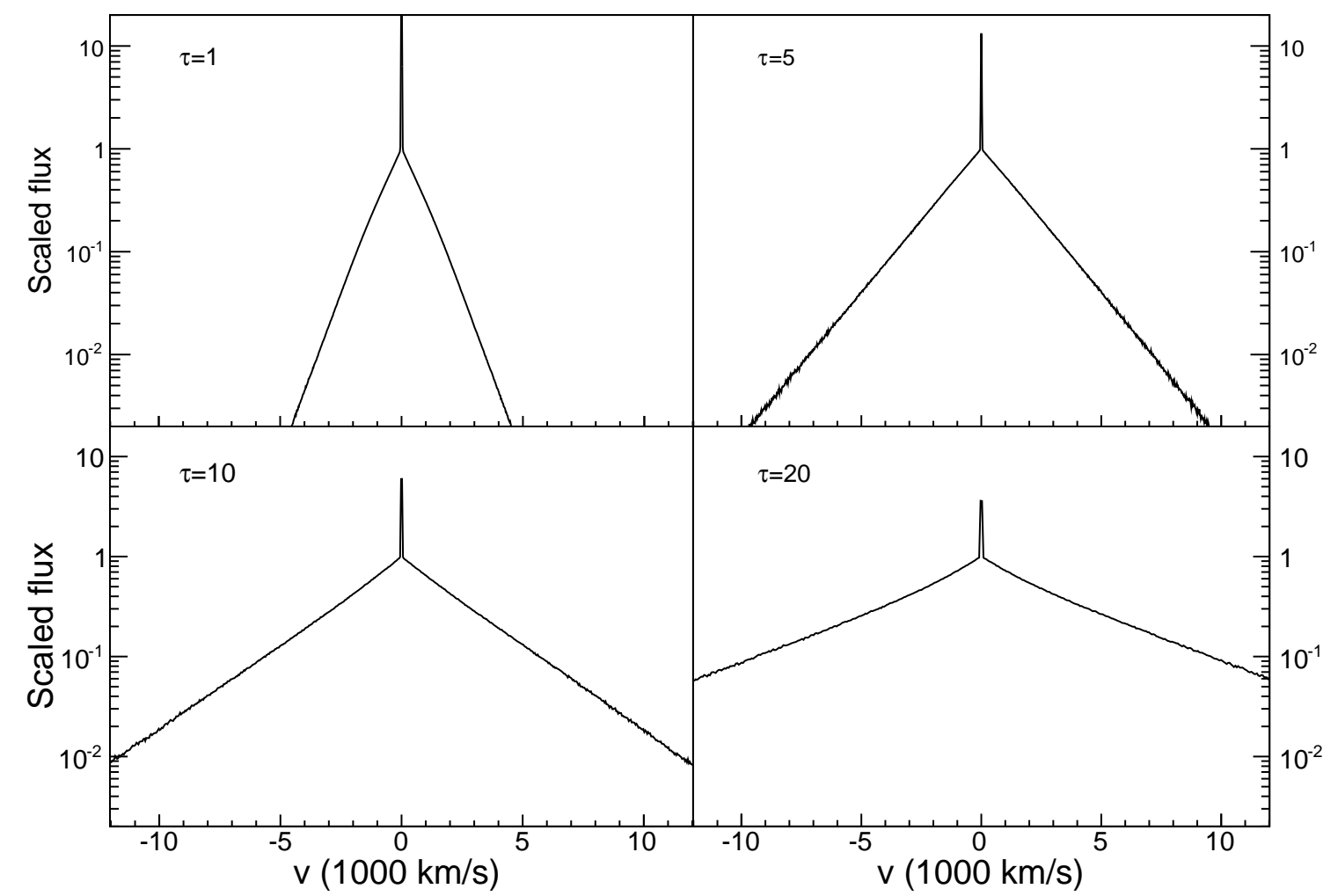

Fig. 3.3. - Line profile results plotted on a log scale, for a circumstellar medium with $T=20,000 \mathrm{~K}, n \propto r^{-2}, R_{2} / R_{1}=10^{3}$, and a static medium. The optical depths $\tau=1,5,10,20$ are shown.

of narrow to broad component depends on the escape probability of the photons and is shown in Fig. 3.7. We also give the approximate expression for the escape probability $[1-\exp (-\tau)] / \tau$; it can be seen that the accurate value is somewhat below this estimate. The approximate expression is only accurate under the assumption that the photons are confined to move in the radial direction. An assumption made in these simulations is that the narrow line component is optically thin to the emission. In Type IIn supernovae, the narrow $\mathrm{H} \alpha$ component frequently shows optical depth effects, i.e. a P Cygni line profile (e.g., Kiewe et al. 2012), so the present considerations do not apply. However, the results for the narrow line are potentially applicable to higher level $\mathrm{H}$ lines and other lines with lower optical depth. 


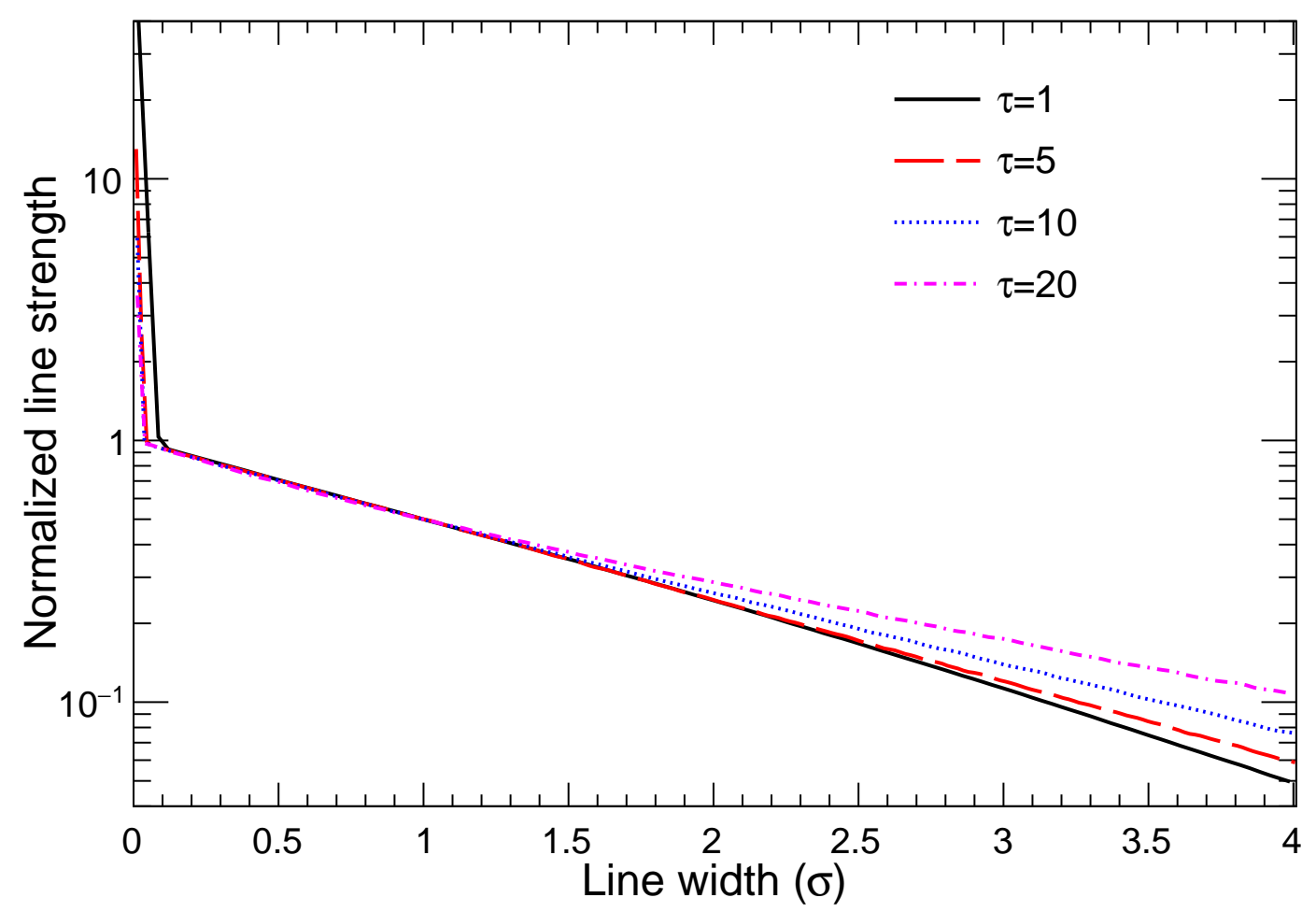

Fig. 3.4.- Normalized line shape, with the lines folded about 0 velocity. An $n \propto r^{-2}$ density profile is assumed and $R_{2} / R_{1}=10^{3}$. The $x$-axis, $\sigma$, is the line half width in units of the half width half maximum, and the $y$-axis is normalized by the peak of the broad component.

We have assumed a density profile that is appropriate to a steady wind from the progenitor star. However, the mass loss processes that give rise to the dense circumstellar medium around a Type IIn supernova are not understood and are likely to be complex. In order to check the sensitivity to the density profile, we undertook simulations with a density profile $\rho \propto r^{-s}$, with $s$ in the range $0-3.7$ and $\tau=5$. The FWHM and narrow to total line ratio are shown as a function of $s$ in Figs. 3.8 and 3.9. It can be seen that, for the narrowest scattering layer, there is the least dependence on $s$. In the limit that the layer is geometrically thin, the results are expected to be independent of $s$. For $s=2$, already discussed, the insensitivity of the results to 


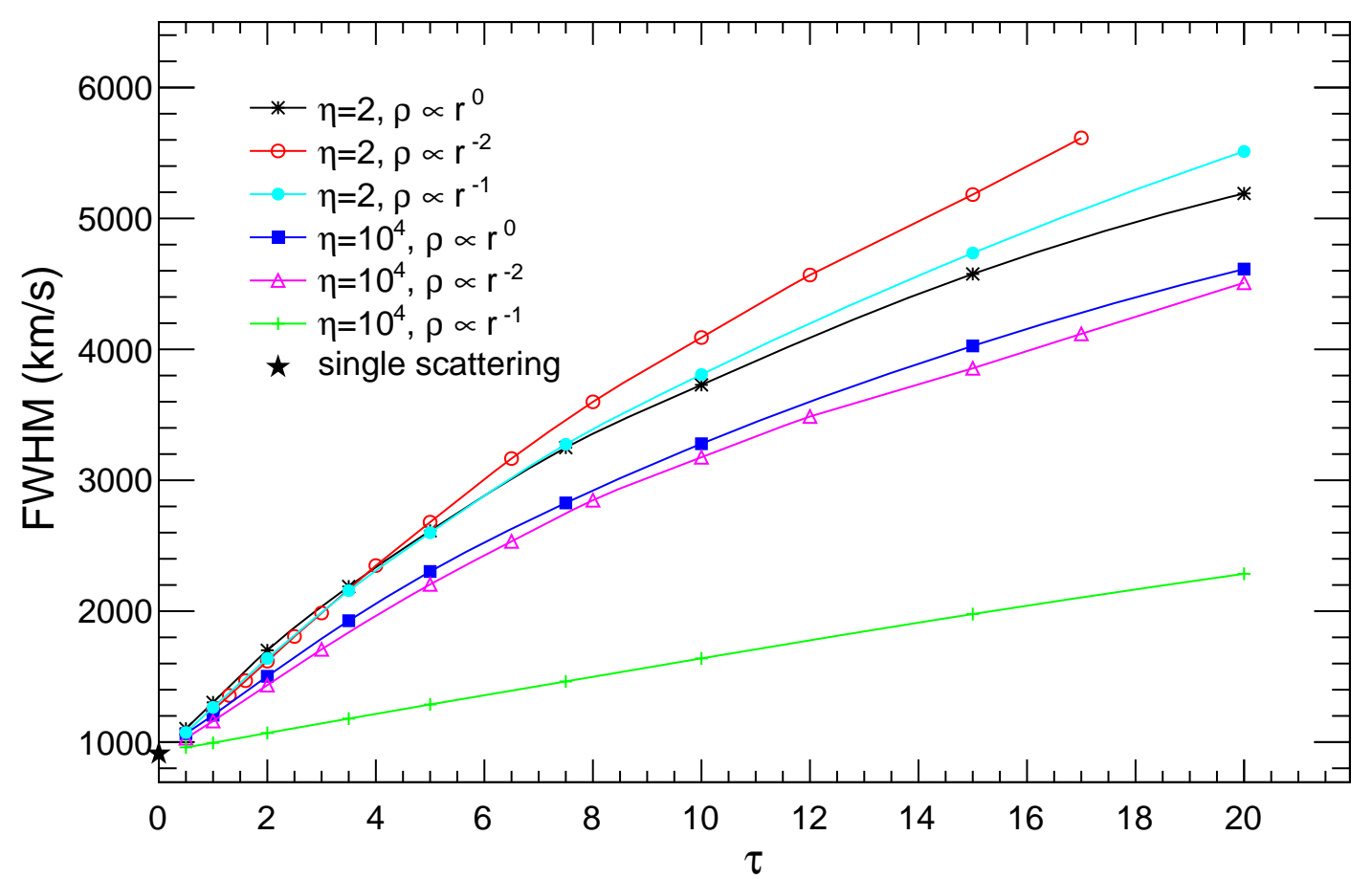

Fig. 3.5.- Relation of FWHM vs. $\tau$, assuming $T=20,000 \mathrm{~K}, n \propto r^{-2}, r^{-1}, r^{0}$, and two different radial extents $\eta=R_{2} / R_{1}$. For comparison, the FWHM of the single scattering case, $913 \mathrm{~km} \mathrm{~s}^{-1}$, is shown as black star.

the radial extent is clear; most of the contribution to scattering comes from layers that are close to $R_{1}$. The same is true for $s>2$. On the other hand, for a radially extended region with $s=0$, most of the scattering occurs in layers close to $R_{2}$. The results do not depend significantly on the position of $R_{1}$ provided that the region is radially extended. In comparing line profiles for the $s=0$ case and the $s=2$ steady wind case, we find that the FWHM approximately agrees for the two cases, but that the outer wings of the line profiles are elevated in the constant density case.

It can be seen in Figs. 3.8 and 3.9 that there are significant deviations from the standard results between $s=0$ and 2, which we interpret as follows. The production of photons in a given logarithmic radial range is $\dot{N} \propto 4 \pi R^{2} \Delta R \epsilon$, where $\Delta R \propto R$ and $\epsilon \propto n^{2}$ is the gas emissivity, so that $\dot{N} \propto R^{3-2 s}$. Thus for $s<1.5$, most of the 


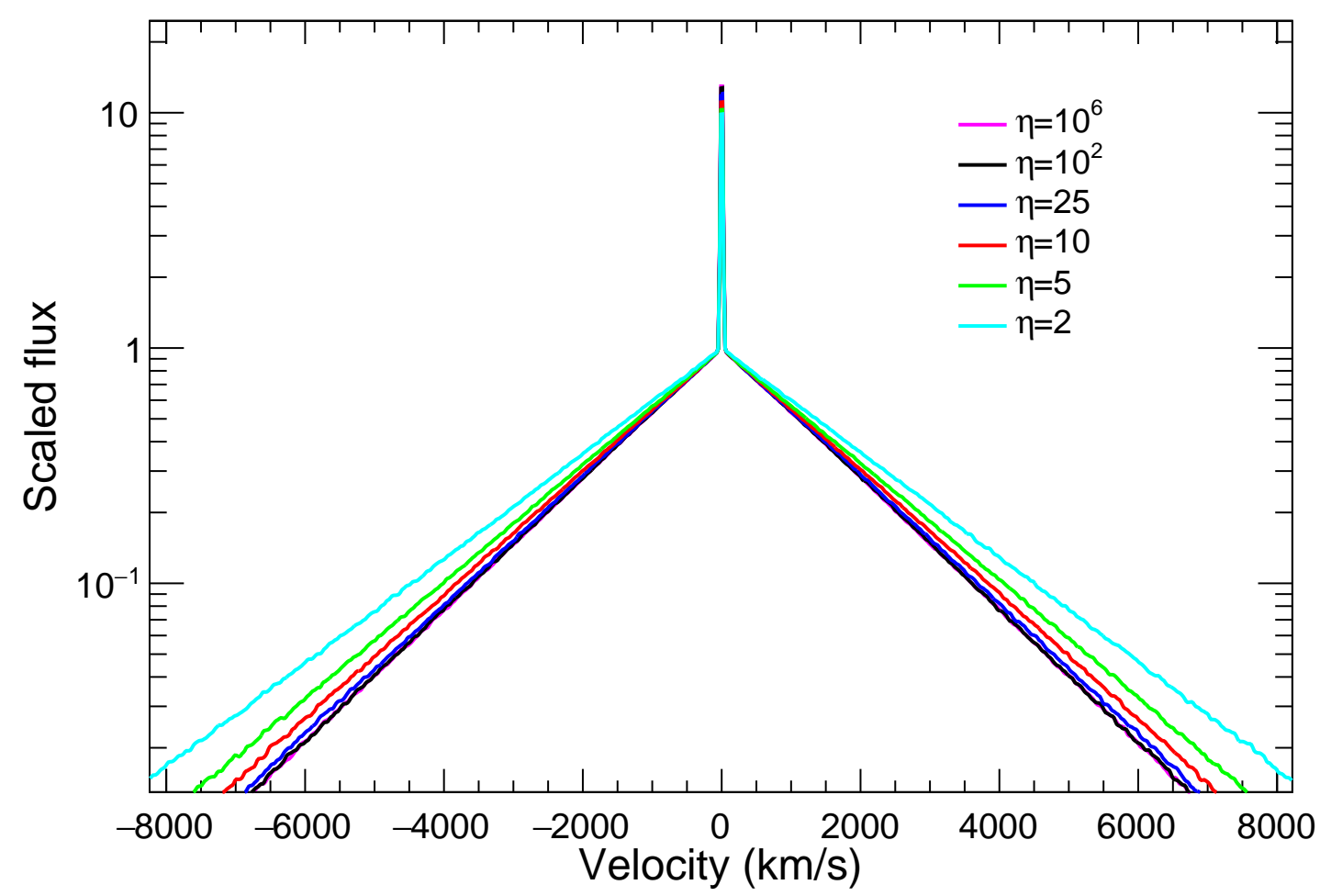

Fig. 3.6. - The line profile for a series of outer to inner radius ratios $\left(\eta=R_{2} / R_{1}\right)$ assuming an $n \propto r^{-2}$ medium.

photons are produced at large radii, while for $s>1.5$, they are produced at small radii. For the optical depth $\tau \sim \int \rho \kappa d r, s=1$ is a critical value above which most of the optical depth is contributed at small radii and below which most is contributed at large radii. The result is that for $s>1.5$ both the photon production and the optical depth effects occur at small radii, while for $s<1$, they both occur at large radii. In the range $1<s<1.5$, most of the photons are produced at large radii, but the electron scattering optical depth occurs at small radii. The result is that the photons can escape more easily without scattering, as is shown in Fig. 3.8. At the same time, the rapid escape of photons leads to a narrower FWHM (Fig. 3.9). 


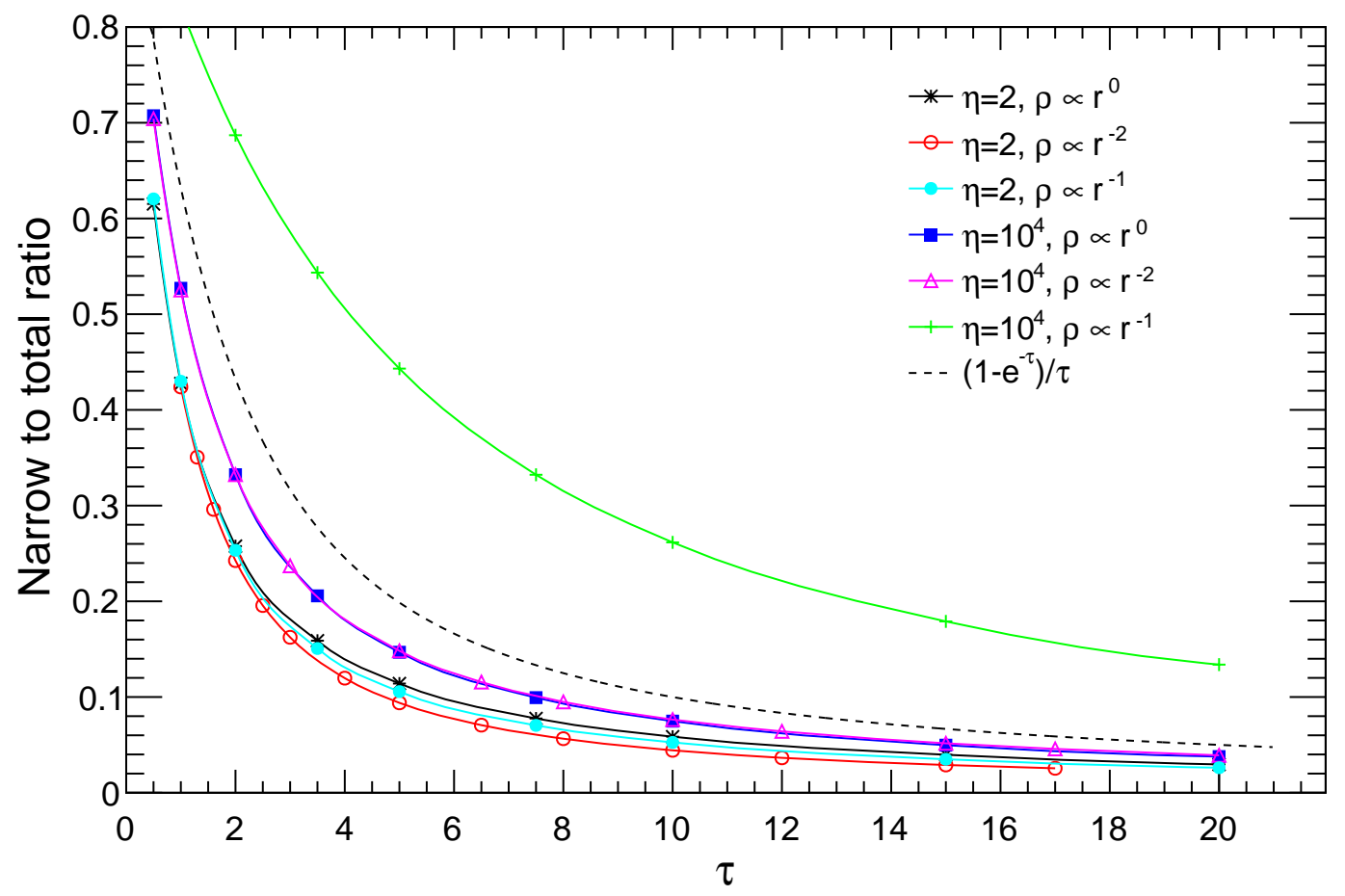

Fig. 3.7.- Relation of narrow to total line ratio vs. $\tau$, assuming $T=20,000 \mathrm{~K}$, $n \propto r^{-2}, r^{-1}, r^{0}$, and two different radial extents $\left(\eta=R_{2} / R_{1}\right)$. The dashed line shows the relation given by the simple analytic formula.

\subsubsection{Presupernova Mass Loss Velocity}

Up to this point, we have assumed that the scattering medium is stationary so that the line broadening is entirely due to the thermal velocities of electrons. In the actual case, the mass loss gas is expected to have some outflow velocity, $v_{w}$, from the parent star. In Fig. 3.10, we show the line profiles for various values of $v_{w}$ for the standard case with $\tau=5, s=2, R_{2} / R_{1}=10$, and $T=20,000 \mathrm{~K}$. It can be seen that even for $v_{w}=200 \mathrm{~km} \mathrm{~s}^{-1}$ there is a some asymmetry introduced into the broad line profile, even though the thermal velocity of the electrons is $954 \mathrm{~km} \mathrm{~s}^{-1}$. We attribute the effect to the fact that the wind velocity gives a systematic redshift to the scattering photons, while the thermal velocities are equally positive and negative resulting in 


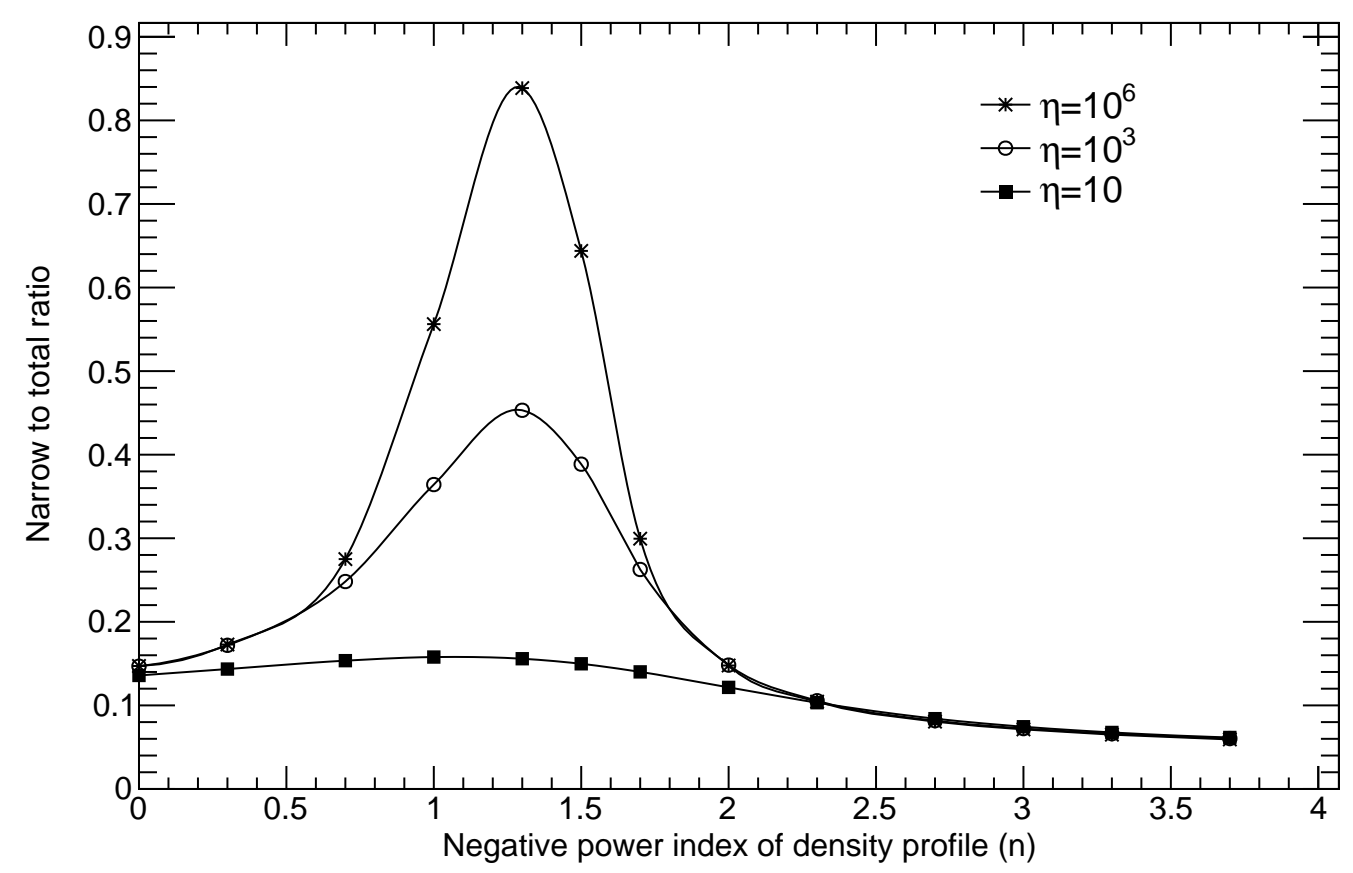

Fig. 3.8. - Narrow to total line ratio vs. the power law of the density profile for 3 different sets of radial ranges $\left(\eta=R_{2} / R_{1}\right)$, assuming $\tau=5$ and $T=20,000 \mathrm{~K}$. Here, $\eta$ is the ratio of outer radius to inner radius for the scattering region.

a diffusion in frequency. The systematic redshift is due to the spherical divergence of the flow. In the radial direction, velocity is constant, so there is no systematic change in photon energy. For scattering in a nonradial direction, there is a velocity component away from the photon source that gives a systematic redshift. A similar situation occurs for electron scattering in the winds of Wolf-Rayet stars, although in this case there may be a region where the wind accelerates in the radial direction to its final velocity (Hillier 1991); the acceleration is expected to give rise to a systematic redshift of scattering photons. As can be seen in Fig. 3 of Hillier (1991), the red wing of the scattered component is considerably stronger than the blue wing.

In the present case, there is no velocity gradient in the radial direction, so that scattering away from a radial line is important for causing an asymmetry. In addition, 


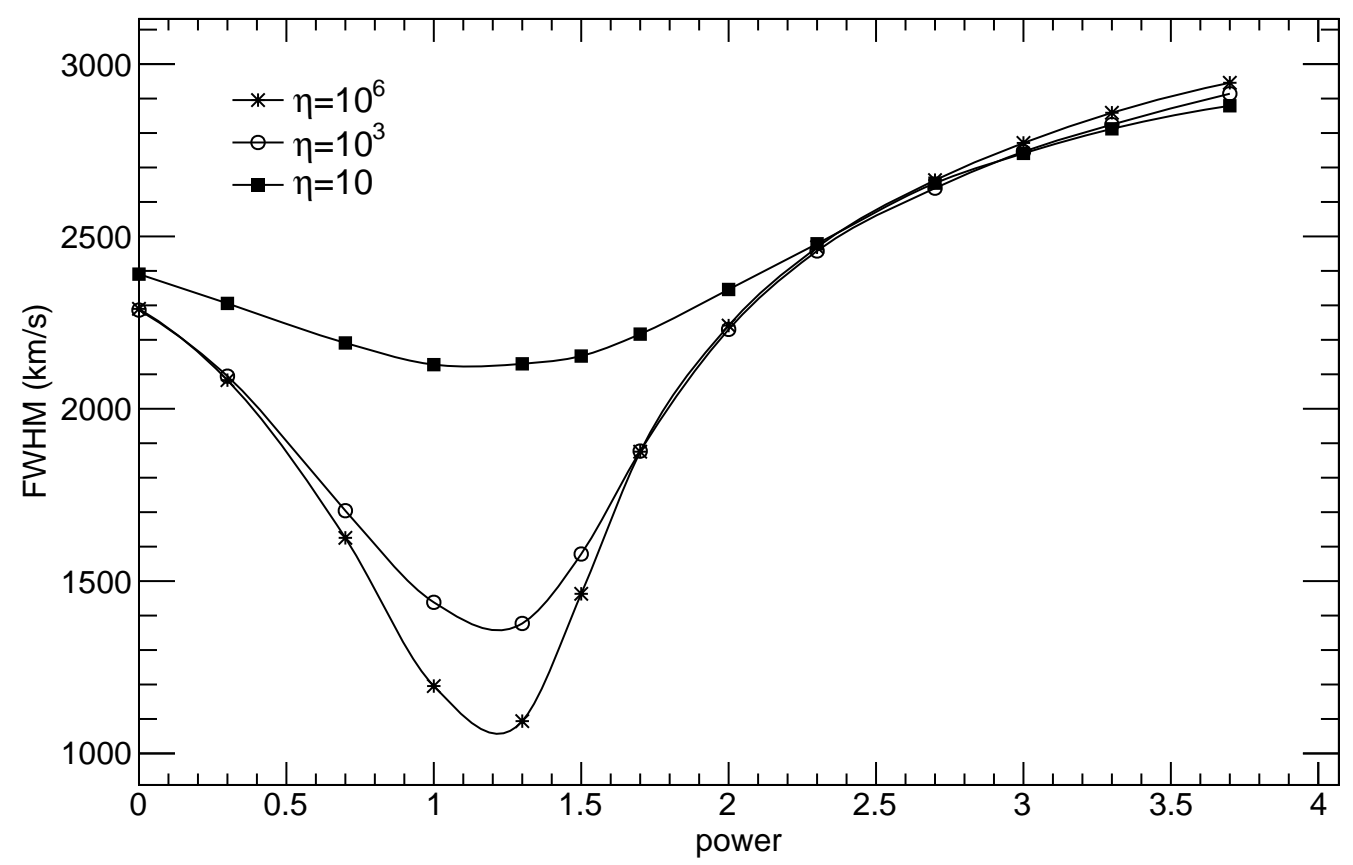

Fig. 3.9.- Same as Fig. 3.8 but for the FWHM.

scattering at a great distance from the photon source gives rise to a greater velocity difference and redshift. Thus the case of $n \propto r^{-2}$ in an extended scattering region is especially favorable for producing an asymmetry. In addition, photons are emitted at a deeper optical depth on average for a thicker scattering region, which could also contribute to the asymmetric profile. If the scattering layer is narrow, the photons do not move far in angle before escaping and thus the asymmetry is small. For the case studied by Chugai (2001), $R_{1}=4 \times 10^{14} \mathrm{~cm}$ and $R_{2}=9 \times 10^{14} \mathrm{~cm}$, so the narrow scattering region gives rise to a negligible asymmetry; the wind velocity was taken to be $40 \mathrm{~km} \mathrm{~s}^{-1}$. The asymmetry of the narrow peak weakens with increasing thickness of scattering layer because fewer non-scattered (and redshifted) photons are absorbed by the inner boundary.

We considered the effect of the density power law index for the scattering gas $(\rho \propto$ 


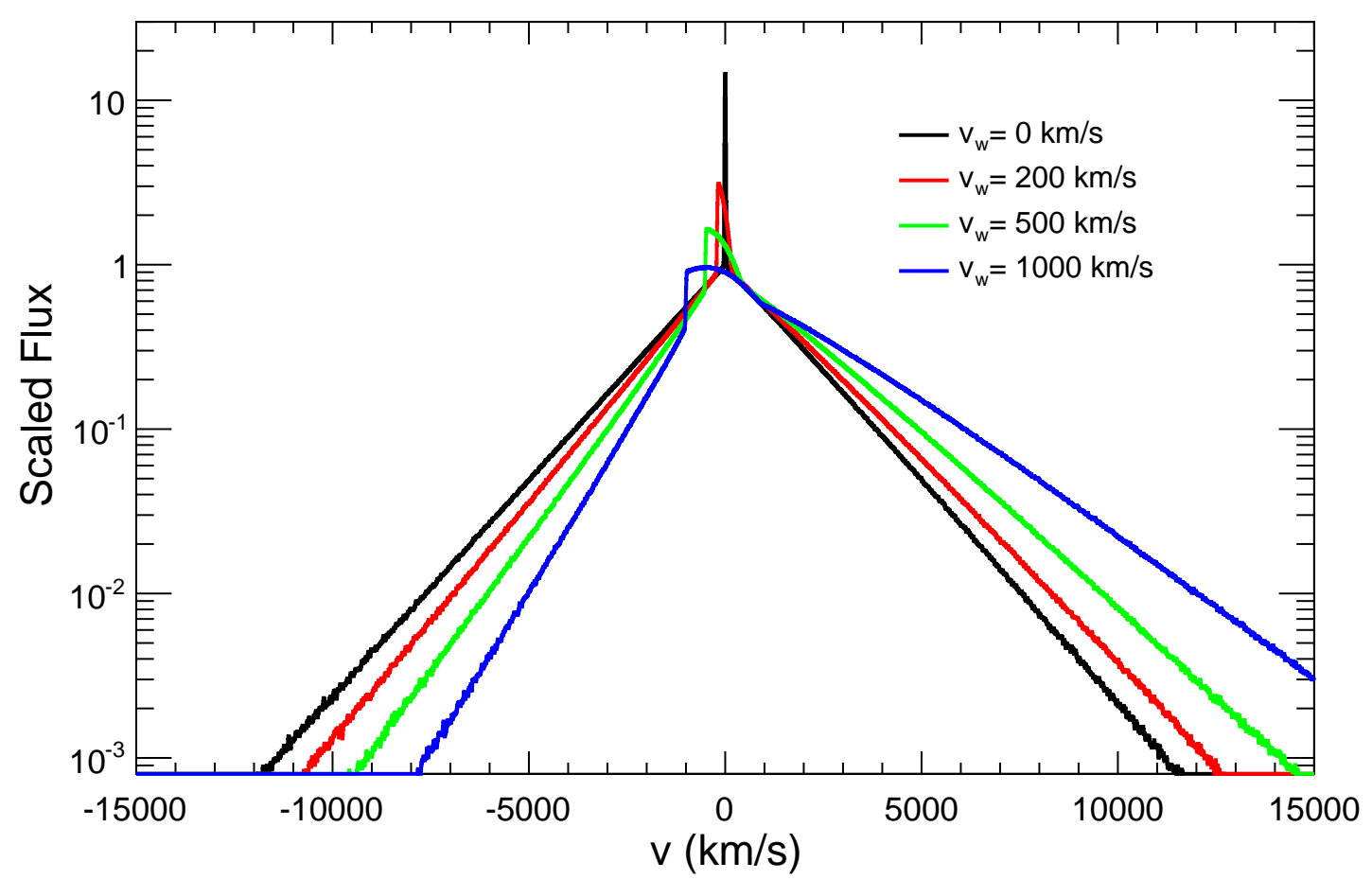

Fig. 3.10.- Line profiles for $n \propto r^{-2}, \tau=5, R_{2} / R_{1}=10, T=20,000$, and various values of the wind velocity $v_{w}$, with constant outflow velocity.

$\left.r^{-2}, r^{-1}, r^{0}\right)$. For the broad component, the degree of asymmetry is comparable for the 3 density profiles. The far blue wing becomes stronger for a flatter density profile. For the uniform density case, most photons are created near the outer boundary. Because of the density profile, even photons that escape horizontally must penetrate a large optical depth, so there are fewer narrow line photons at zero velocity.

We investigated variations in the optical depth. To compare the asymmetry of the profile with different optical depths, different temperatures were chosen in order for the broad components to have the same width. The value of $v_{w}$ was also scaled with $\sqrt{T}$ correspondingly. If the medium is thick, the asymmetry is insensitive to the optical depth. However, if the medium is thin, for a larger optical depth, the asymmetry is slightly smaller because the photon cannot travel far in angle between 
two scatterings.

We also considered the line profiles in the linear velocity profile case where $v_{w}=$ $v_{b}\left(r / R_{2}\right)$, as might occur in explosive mass loss. Here, $v_{b}$ corresponds to the velocity at the outer boundary. This explosive mass loss scenario has been suggested for SN 2006gy (Smith et al. 2010). The profile is similar to a thin layer constant wind velocity case with a smaller optical depth. This is because the profile is dominated by the medium with the largest velocity which is in a relatively thin layer near the outer boundary. Except for the shape of the narrow component, the profile is insensitive to the thickness of the layer.

Because a thinner medium leads to a smaller asymmetry for the same expansion velocity, a larger wind velocity is required in fitting the same spectrum. As a result, the profile of a thinner medium shifts to the blue more than a thicker medium which has the same asymmetry. The sharp jump at the blue edge of the narrow component would be smeared out by the Gaussian convolution in an actual observed profile.

If the scattering medium is stationary, the top of the broad component has a sharp tip, like the shape shown in the single scattering profile (Fig. 3.2). When scattered by an expending medium, the broad component has a rounded top instead, shown as the red dotted line in Fig. 3.13. The rounded top becomes wider and lower as the wind velocity increases. Because the width of the rounded top is always narrower than the narrow component, it does not show up in the overall spectrum. However, when evaluating the FWHM of the broad component, which depends on the maximum value of the broad component; a larger wind velocity leads to a larger FWHM measurement. 


\subsubsection{Radiative Acceleration of Circumstellar Gas}

When a shock wave breaks out of the progenitor star, the radiation dominated shock transition becomes broad as the radiation is able to diffuse away from the star. The strong radiation field is expected to accelerate the circumstellar medium with a velocity profile $\propto r^{-2}$ because of the spherically diverging radiation flux. Chugai (2001) used a velocity profile

$$
v(r)=v_{w}+v_{s h}\left(\frac{R_{1}}{r}\right)^{2},
$$

where $v_{w}$ is the velocity of the presupernova wind and $v_{s h}$ is the velocity from acceleration at the inner boundary $R_{1}$.

Some insight into the effect of $v_{s h}$ can be obtained from examination of the $\tau=1$ case. Fig. 3.11 shows the profile with $\eta=10$ and $T=125,000 \mathrm{~K}$. The reason for choosing this unphysically high temperature is to match its width with the larger optical depth case shown in Fig. 3.10. The line profile is substantially affected out to $v_{s h}$, especially on the blue side because of occultation effects on the red side (see the $v_{s h}=2000 \mathrm{~km} \mathrm{~s}^{-1}$ case). An inflection appears at the connection point between the region dominated by the narrow component and by the broad component. As noted by Chugai (2001), the effects become especially significant for $v_{s h}>1000 \mathrm{~km} \mathrm{~s}^{-1}$. In the velocity range $|v|<v_{s h}$, the main contribution is from unscattered photons and there is an asymmetry to the blue. For $|v|>v_{s h}$, the flux is dominated by scattered photons, whose properties are primarily determined by the thermal velocities of electrons. If the optical depth $\tau>2$, the narrow component smoothly transitions to the broad component and the inflection point disappears because the broad component takes over at a smaller $|v|$.

A larger $v_{s h}$ can tilt the narrow component to the red, because most unscattered photons are emitted in a small optical depth region that is close to the outer boundary 


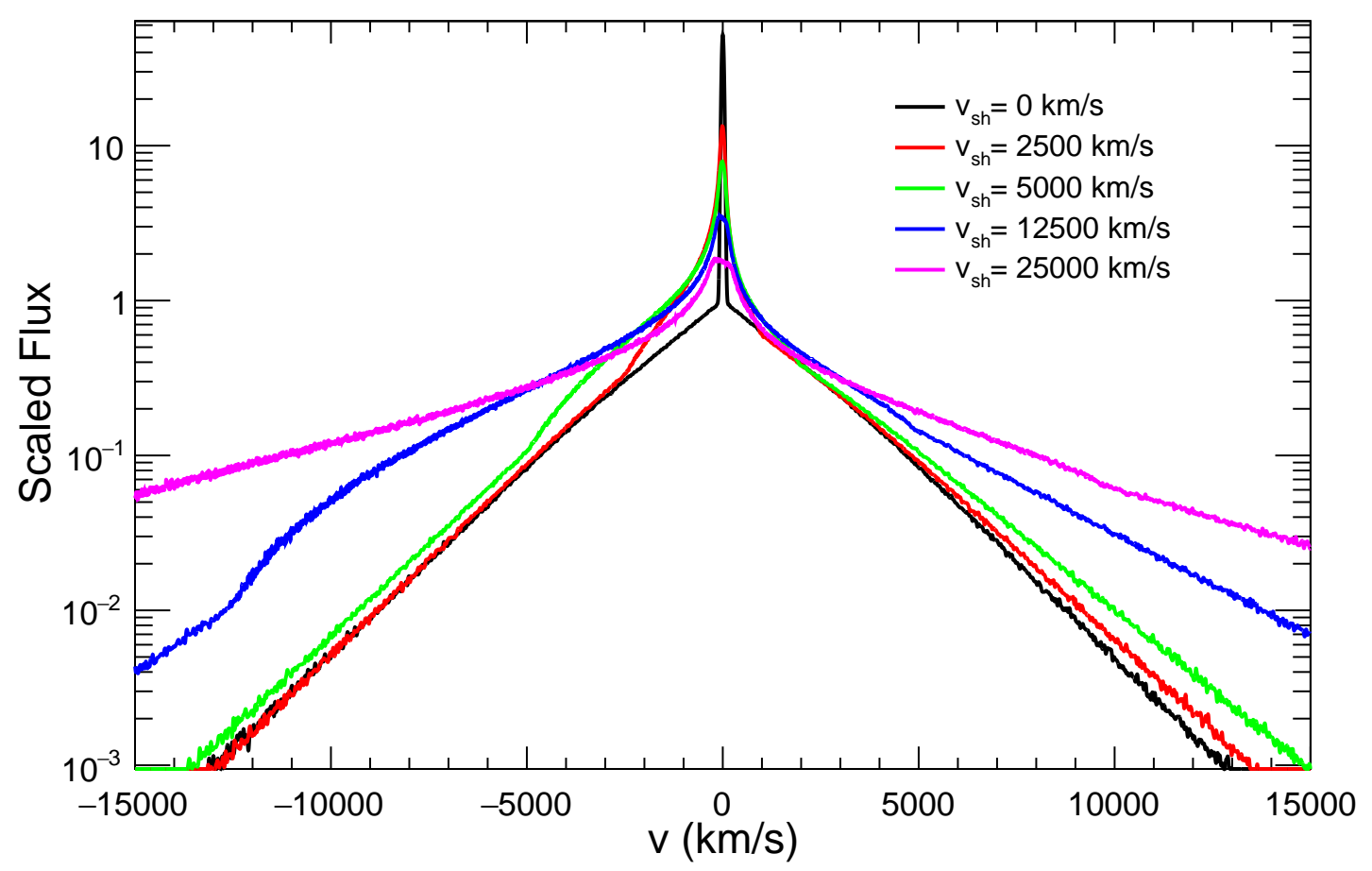

Fig. 3.11. - Line profiles for $n \propto r^{-2}, \tau=1, R_{2} / R_{1}=10, T=125,000$, and various values of the preshock velocity $v_{s h}$ determined by radiative acceleration of the gas. The wind velocity $v_{w}=0$.

where the expansion velocity is small. This effect is less dramatic if the optical depth is small or the radius ratio is small.

For a geometrically thin layer, all the gas is fast moving, so the non-scattered component is broad. For a thick layer, the outer part of the medium is almost static so the narrow component has a sharp peak (Fig. 3.11). Especially when $\tau>1$, few photons near the inner boundary can escape without scattering. The geometrical thickness has a small effect on the broad profile for a thick layer $(\eta \gtrsim 3)$. On the one hand, the outer part of the thick layer moves very slowly; on the other, a thick layer leads to a stronger spherical divergence effect and more photons are emitted at a deeper optical depth. These two effects basically cancel each other.

For a thick layer with uniform density or an $r^{-1}$ density profile, most photons are 
emitted in the outer part of the medium, where the velocity is small if there is no wind velocity. All the profiles are the same. If the medium is not extremely thick (e.g., $R_{2} / R_{1}=10$ ), both blue and red line wings can be stronger, as in the case of a $r^{-2}$ density profile.

\subsubsection{Effects of Continuum Absorption}

The calculations in the previous section assume only scattering, with no absorption. In order to estimate the effect of absorption, we introduced an absorption probability parameter that is defined as the probability that a photon is absorbed for each collision event. Fig. 3.12 shows that the effect of continuum absorption is very similar to a smaller optical depth with no absorption. We note that continuum absorption does not give rise to a weakening of red emission, as typically occurs in the supernova case because of absorption of emission from the receding part of the explosion. Here, the line broadening is due to the thermal velocities of the electrons and not to the overall expansion.

In the absence of dust, the relevant absorption processes are free-free and boundfree absorption. As discussed by Chugai (2001), both of these are expected to be small for the physical conditions of interest.

In principle, dust is also a possible source of continuum absorption and has been suggested as the source of an asymmetric broad line in SN 2010jl (Smith et al. 2012; Gall et al. 2014). As discussed above, this would require that the explosive motions play a role in the line broadening. In addition, at early times the radiation and gas temperatures remain $>5000 \mathrm{~K}$ so that the conditions are not appropriate for the formation of dust. Fransson et al. (2014) have discussed the issues with inferring the presence of dust in the supernova. 


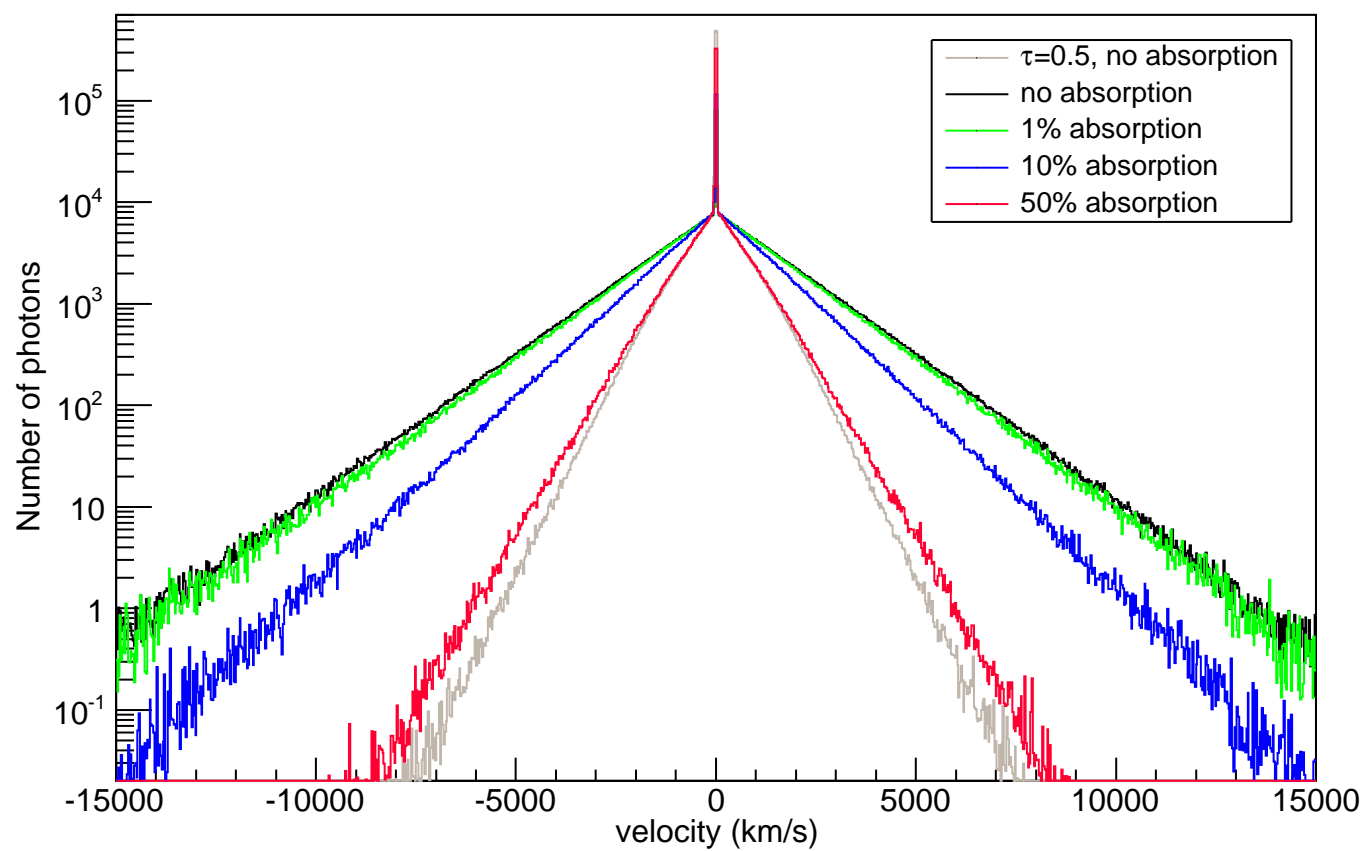

Fig. 3.12.- The variation of the line profile with continuum absorption, assuming $\tau=5, T=20,000 \mathrm{~K}, n \propto r^{-2}$, and a stationary circumstellar medium. The line profile for $\tau=0.5$ is shown for comparison.

\subsection{Comparison with Observations}

The models calculated here were compared to observed supernova line profiles, which were downloaded from the WISeREP repository of supernova spectra (Yaron \& GalYam 2012). Spectra of Type IIn supernovae were chosen that had a well-observed H line. The supernova spectra that were used are listed in Table 3.1 and are shown in Figs. 3.13 - 3.23. The ages given in Table 3.1 are from the time of discovery. We chose the $\mathrm{H}$ lines which have the best data available and a smooth continuum. There can be interference on the red side of the line with the He I $\lambda 6678$ line, which is at a wavelength corresponding to $\sim 5300 \mathrm{~km} \mathrm{~s}^{-1}$ relative to $\mathrm{H} \alpha$. The $[\mathrm{N} \mathrm{II}] \lambda 6611$ and [N II] $\lambda 6482$ emission lines may also present on the red and blue side of the $\mathrm{H} \alpha$ 
respectively, corresponding to $\sim 2200 \mathrm{~km} \mathrm{~s}^{-1}$ and $\sim 3700 \mathrm{~km} \mathrm{~s}^{-1}$ relative to $\mathrm{H} \alpha$; the [N II] lines are probably from the host galaxy. The references in Table 3.1 are to the papers that presented the spectra we are using. Where possible, we use the blackbody temperature estimates at those times to determine the background emission.

An issue with modeling the $\mathrm{H} \alpha$ line is that it commonly shows a narrow $\mathrm{P}$ Cygni line profile in the spectra of Type IIn SNe, showing that there are line optical depth effects in the narrow line. Our models assume an optically thin medium and thus are primarily directed at the broad (scattered) line component. We have chosen supernovae in which the $\mathrm{H} \alpha$ line shows distinct broad and narrow components.

The models were calculated as follows. The scattering occurs in the circumstellar medium outside of the supernova shock wave. The gas is thus heated and ionized by the energetic radiation from the postshock region. We considered a spherically symmetric isothermal circumstellar medium with a $r^{-2}$ density profile and a velocity profile described by Equation (3.1). We preferred a uniform expanding velocity, or $v_{s h} \approx 0$ in the fits, especially for the spectra at late time, because radiative acceleration is important around the time of shock breakout, which occurs at an early phase. The $\mathrm{H} \alpha$ photons are emitted by the same medium due to radiative recombination, so the emissivity is proportional to the density squared.

The fitting parameters considered were gas temperature $T, \tau, \eta, v_{w}, v_{s h}$ and redshift $z$. The calculated emission lines were convolved with Gaussian profiles with FWHM equal to the spectral resolution. The preferred fitting parameters are listed in Table 3.2. The listed FWHMs are the measured values for the broad components of the comparison models. Because the maximum value of the broad component is extrapolated from the line wing following the curvature of the comparison model, the measured value is model dependent. The uncertainty in the FWHM is estimated to 
be about of $200 \mathrm{~km} \mathrm{~s}^{-1}$.

The two main model parameters determining the width of the broad component are the electron scattering optical depth $\tau$ and the temperature $T$ of the gas. The line profile by itself cannot be used to determine $\tau$ because sensitivity to $\tau$ generally occurs far out in the wings where the signal-to-noise ratio of the data is low and the uncertain continuum level plays a role. If the line is optically thin, there is a clear mapping from the model $\tau$ and $T$ to the observed line width and the ratio of narrow to broad line flux. As noted abovee, $\mathrm{H} \alpha$ frequently has a P-Cygni profile, which indicates the line formation region is not optically thin and affects the narrow line flux. Therefore, the fitting was focused on the broad component, and $\tau$ can only be loosely constrained by the physical requirements on the gas temperature. For the level of ionization indicated in SNe IIn, a gas temperature $\sim 10,000-20,000$ $\mathrm{K}$ is expected (Kallman \& McCray 1982). Our method depends on being able to separate the broad component from the narrow component of $\mathrm{H} \alpha$. We found that by plotting the line flux on a logarithmic scale, it was generally possible to separate these components because of an inflection at the transition point in the line profiles. The inflection did not stand out on a linear scale. We plot the spectra here on a log scale.

The thickness of the medium $\eta$, expanding wind velocity $v_{w}$ and $v_{s h}$ determine the asymmetry of the broad component, the shift in the peak of the line and the shape of the narrow component. The value of $v_{w}$ suggested by the P-Cygni profile, if available, was used in the fits. We examine some implications of this assumption.

In addition to the scattering calculation, a critical part of the fitting procedure is choosing the continuum level because it is crucial for determining the outer wings of the line. When possible, our fits extend out to a wavelength region where the scattered 
line does not appear to be playing a role. The observed spectra were corrected for the reddening listed in Table 3.1, where the ratio of total to selective extinction $R_{V}=3.1$. The continuum is fit by a blackbody spectrum. In many cases, the full continuum cannot be represented by a single blackbody continuum. In those cases, a blackbody that matches the continuum near the line was subtracted.

Another parameter of the models is the redshift $z$. Because of the effect of the expanding medium, the redshift of the supernova cannot simply be determined by the peak of the narrow component. If the redshift of the supernova is available, e.g. SN 1998S, this redshift is chosen. In most cases, only the redshifts of the host galaxy are available, which can only loosely constrain the redshift of the supernova. Then $z$ was set by the broad component of the spectrum.

\subsubsection{SN 1998S}

SN 1998S was discovered on 1998 March 2.7, which was probably within a few days of the explosion (Fassia et al. 2001). Spectra taken between March 4 and March 7 (Shivvers et al. 2015; Leonard et al. 2000; Fassia et al. 2001) showed evidence for a narrow $\mathrm{H} \alpha$ line with broad wings (Figs. 3.13 and 3.14). We present spectra from March 4 and 5 separately because there was significant evolution of the broad component over that time. For Table 3.1, we took the redshift $z$ at the position of the supernova from Shivvers et al. (2015) because of the high spectral resolution in their observation. The reddening and blackbody temperature are from Fassia et al. (2001). The narrow component in the spectra implied a wind velocity of $40 \mathrm{~km} \mathrm{~s}^{-1}$ (Fassia et al. 2001; Shivvers et al. 2015).

Chugai (2001) developed an electron scattering model for the broad line component in SN 1998S. He fit the H $\alpha$ line in the spectrum from 1998 March 6 (Fassia et al. 
2001). In his Model A, Chugai (2001) took an electron temperature of 21,700 K, as determined from the shape of the optical continuum. The corresponding scattering optical depth was 3.4. The model has an outer to inner radius ratio of $\sim 2$ for the dense scattering region. The limited extent of the dense region is indicated by the light curve of the supernova.

Shivvers et al. (2015) present a high resolution (0.2 A) spectrum of SN 1998S for 1998 March 4. Their Fig. 7 shows the $\mathrm{H} \alpha, \mathrm{H} \beta$ and $\mathrm{H} \gamma$ lines, described by the sum of a narrow Gaussian and a broad modified Lorentzian, where the exponent is allowed to deviate from 2.0. In Fig. 3.13, we model the high resolution observation from 1998 March 4, within 2 days of discovery (Shivvers et al. 2015); note that there are some gaps in the echelle spectrum where a straight line joins the data points. The model parameters are in Table 3.2. The electron scattering model gives a good fit to the observed spectrum. A modified Lorentzian profile with an exponent 2.5 is also shown. The modified Lorentzian roughly agrees with the observed profile out to $2500 \mathrm{~km} \mathrm{~s}^{-1}$ in the line wing, but it bends up further out the line wing, which is not seen in the observed spectrum, and there is a significant difference with the broad electron scattering case at $v=0$. The observed line profile is symmetric, implying that enhanced red emission due to a wind is not present. This can be attributed to 2 factors: the wind velocity, $40 \mathrm{~km} \mathrm{~s}^{-1}$, is much smaller than the thermal velocities of electrons and the extent of the scattering region is relatively small.

In Fig. 3.14, we show fits to the spectra on 1998 March 6 (Leonard et al. 2000) and on 1998 March 6 (Fassia et al. 2001). The latter one is that used by Chugai (2001), The signal-to-noise ratio is higher in the Keck LRIS spectrum with a spectral resolution of $\sim 8 \AA$ (Leonard et al. 2000). The FWHM of the Gaussian smooth function $\left(\sigma=110 \mathrm{~km} \mathrm{~s}^{-1}\right)$ is consistent with the spectral resolution of the observation. 


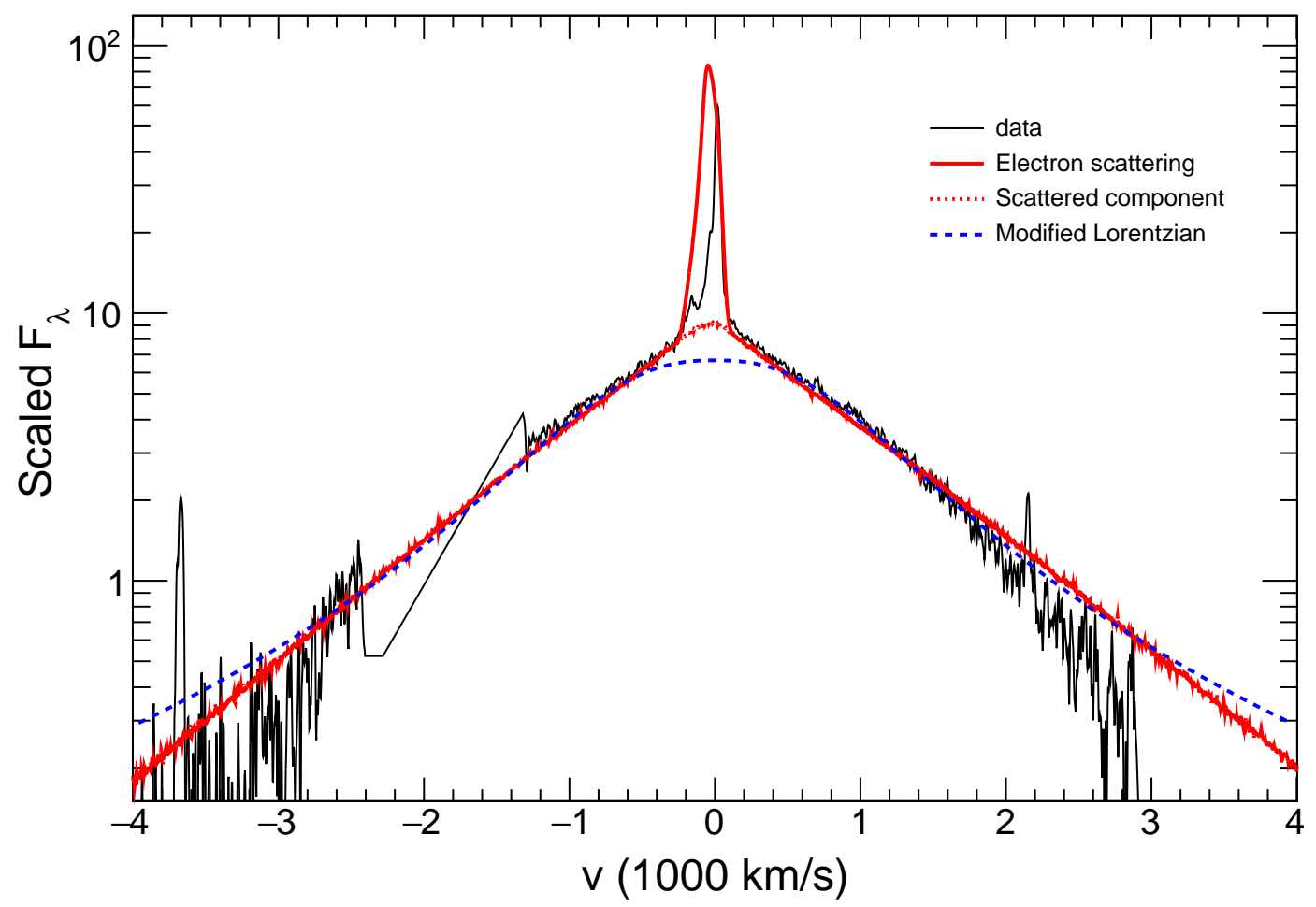

Fig. 3.13. - Comparison of the Monte Carlo electron scattering model result and a modified Lorentzian profile with the SN 1998S H $\alpha$ emission line on 1998 March 4 (Shivvers et al. 2015). The narrow lines near $+2200 \mathrm{~km} \mathrm{~s}^{-1}$ and $-3700 \mathrm{~km} \mathrm{~s}^{-1}$ are due to $[\mathrm{N} \mathrm{II}] \lambda 6611$ and $[\mathrm{N}$ II] $\lambda$ 6482. See the text and Table 3.2 for details.

The figures show that the electron scattering model accounts well for the line profile, as deduced by Chugai (2001). The profiles show the approximate exponential shape that is characteristic of electron scattering, as discussed in Section 2. The results of the fitting are in Table 3.2. Most notable is the large increase in the FWHM of the broad $\mathrm{H} \alpha$ component from day 2 to day 4, which requires an increase in scattering optical depth and/or an increase in electron temperature. Rapid evolution is also present in the He I $\lambda 6678$ line; no broad component is present on March 4, but it is present on March 6 (Figs. 3.13 and 3.14). Shivvers et al. (2015) note that the He I lines show no trace of broad wings on March 4 although they are as strong 


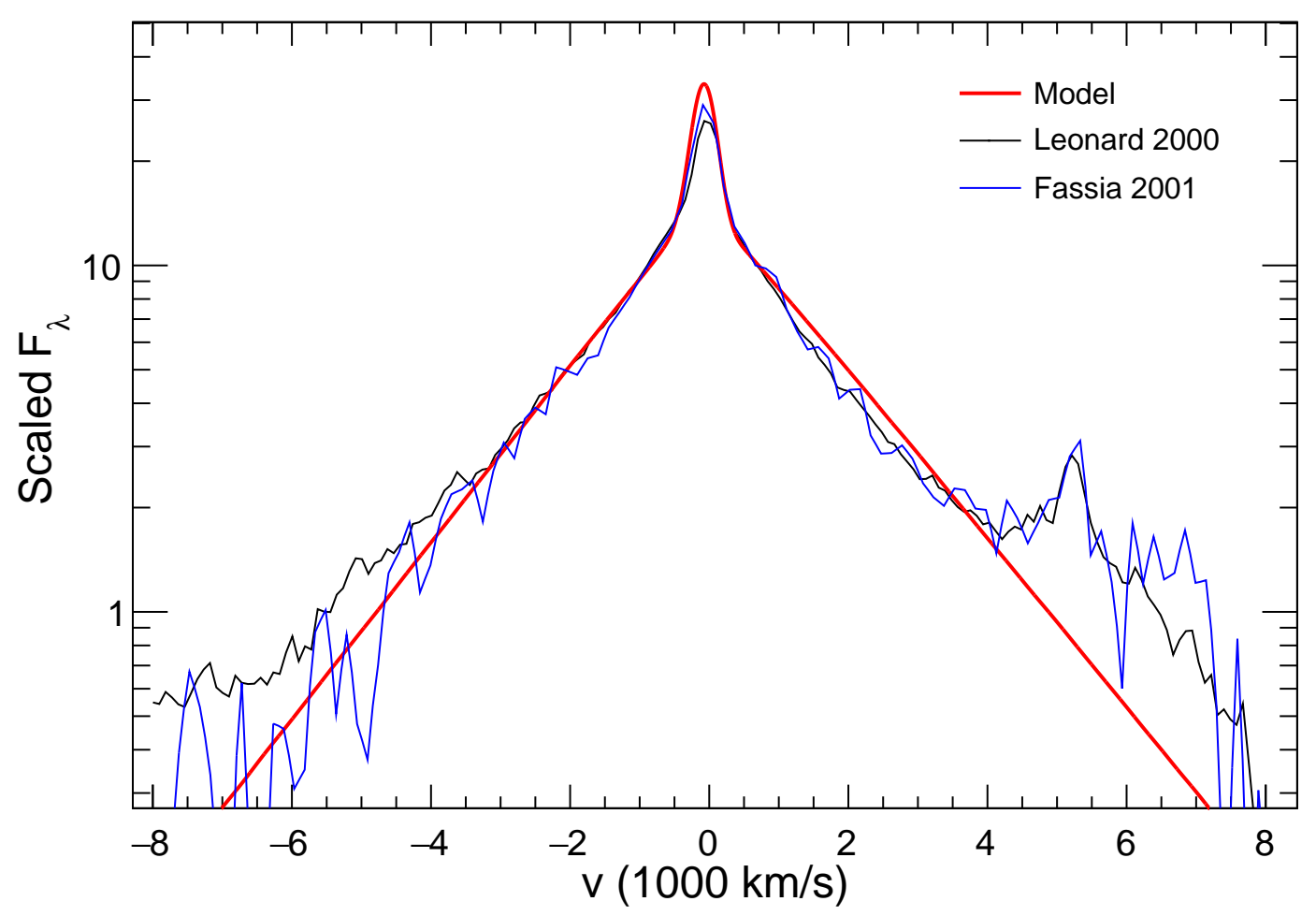

Fig. 3.14. - Comparison of the electron scattering model result with the SN 1998S $\mathrm{H} \alpha$ emission line on 1998 March 6 (Leonard et al. 2000). The He I $\lambda 6678$ line is present, with a broad component.

as the He II line, which does show a broad feature.

Fig. 3.14 shows that the blue wing is somewhat higher in the Leonard et al. (2000) spectrum than in the Fassia et al. (2001) spectrum, although the spectra are close in time. A possibility is that the line profile showed rapid evolution. We found that the excess blue emission could not be fitted in the context of an electron scattering model. As a possible source of the emission, we note that some $\mathrm{H} \alpha$ photons from the $\mathrm{H} \alpha$ shock region of the supernova may be able to escape through the circumstellar envelope. At later times, there is shock emission extending to the blue by $\gtrsim 4,000 \mathrm{~km} \mathrm{~s}^{-1}$ (Fassia et al. 2001). 


\subsubsection{SN 2005cl, SN 2005db, and SN 2012bq}

These events have some properties in common: high FWHM, high wind velocity from P Cygni line, and relatively high redshift. The Type IIn supernovae SN 2005cl and SN 2005db were observed as part of the Caltech Core Collapse supernova Project (CCCP), which followed up on every core collapse supernova observable from Palomar Observatory during the time of the project (Kiewe et al. 2012). The observed events may thus be typical of Type IIn supernovae. We include SN 2012bq in this group because it has similar properties.

A spectrum of SN 2005cl obtained on 2005 July 16, 44 days after discovery (Kiewe et al. 2012), is shown in Fig. 3.15. The spectrum shows a P Cygni profile in the narrow H $\alpha$ line, which Kiewe et al. (2012) take to indicate an unshocked wind velocity of $1318 \pm 223 \mathrm{~km} \mathrm{~s}^{-1}$. Two models for SN 2005cl were developed and are shown in Fig. 3.15. The first one was intended to fit the whole spactrum, including the narrow $\mathrm{H} \alpha$ component. An acceptable fit is obtained but the electron temperature of $80,000 \mathrm{~K}$ is unreasonably high, so we also give a fit with a cool electron temperature. In that case, the narrow component is not well fit, but our model is not intended to fit the narrow component. Also, the electron scattering optical depth is high, $\sim 5$.

A spectrum of SN 2005db was obtained on 2005 Aug 14, 36 days after discovery (Kiewe et al. 2012), see Fig. 3.16. The spectral resolution was $\sim 5 \AA$. The spectrum shows a P Cygni profile in the $\mathrm{H} \alpha$ line, which Kiewe et al. (2012) take to indicate an unshocked wind velocity of $1113 \pm 65 \mathrm{~km} \mathrm{~s}^{-1}$. For SN 2005db, the observed profile does not show a clear turning point at the joint of narrow component and broad component, which introduces some uncertainty into our modeling. SN 2012bq was discovered on 2012 March 30 (Drake et al. 2012). It was studied as part of the PESSTO project SSDR1 (Spectroscopic Survey Data Release 1) and is listed in 


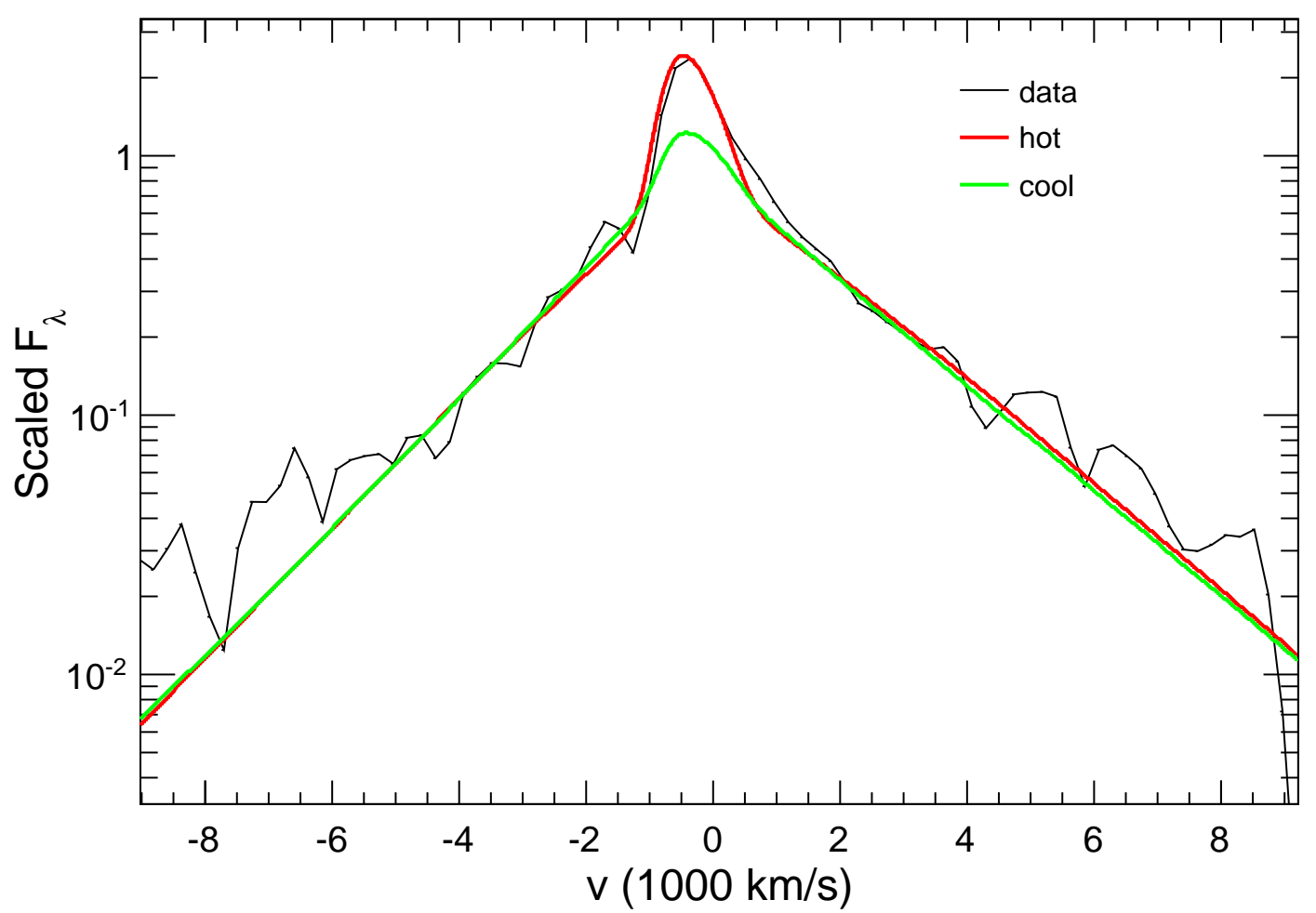

Fig. 3.15. - Comparison of the electron scattering model result with the SN 2005cl $\mathrm{H} \alpha$ emission line on 2005 July 16 (Kiewe et al. 2012). The parameters for the two models are given in Table 3.2.

WISeREP also as LSQ12bry. The observation in Fig. 3.17 is from 2012 Apr 11 with the NNT $3.58 \mathrm{~m}$ telescope, $\sim 18 \AA$ resolution.

The situation is similar for these 3 supernovae. To have a reasonable electron temperature, the narrow component is poorly fit and the value of $\tau \sim 5-6$. In all 3 cases, there is some asymmetry of the broad component, with stronger emission on the red side. As discussed in Section 3.2.3, the asymmetry can be due to scattering in the outflowing wind. In all 3 supernovae there is evidence for a wind with speed $\sim 1000 \mathrm{~km} \mathrm{~s}^{-1}$, which is consistent with what is needed to fit the observations. While there is asymmetry, the line shape of the broad component is still exponential.

A possible problem with this scenario is that of the 4 Type IIn supernovae that 


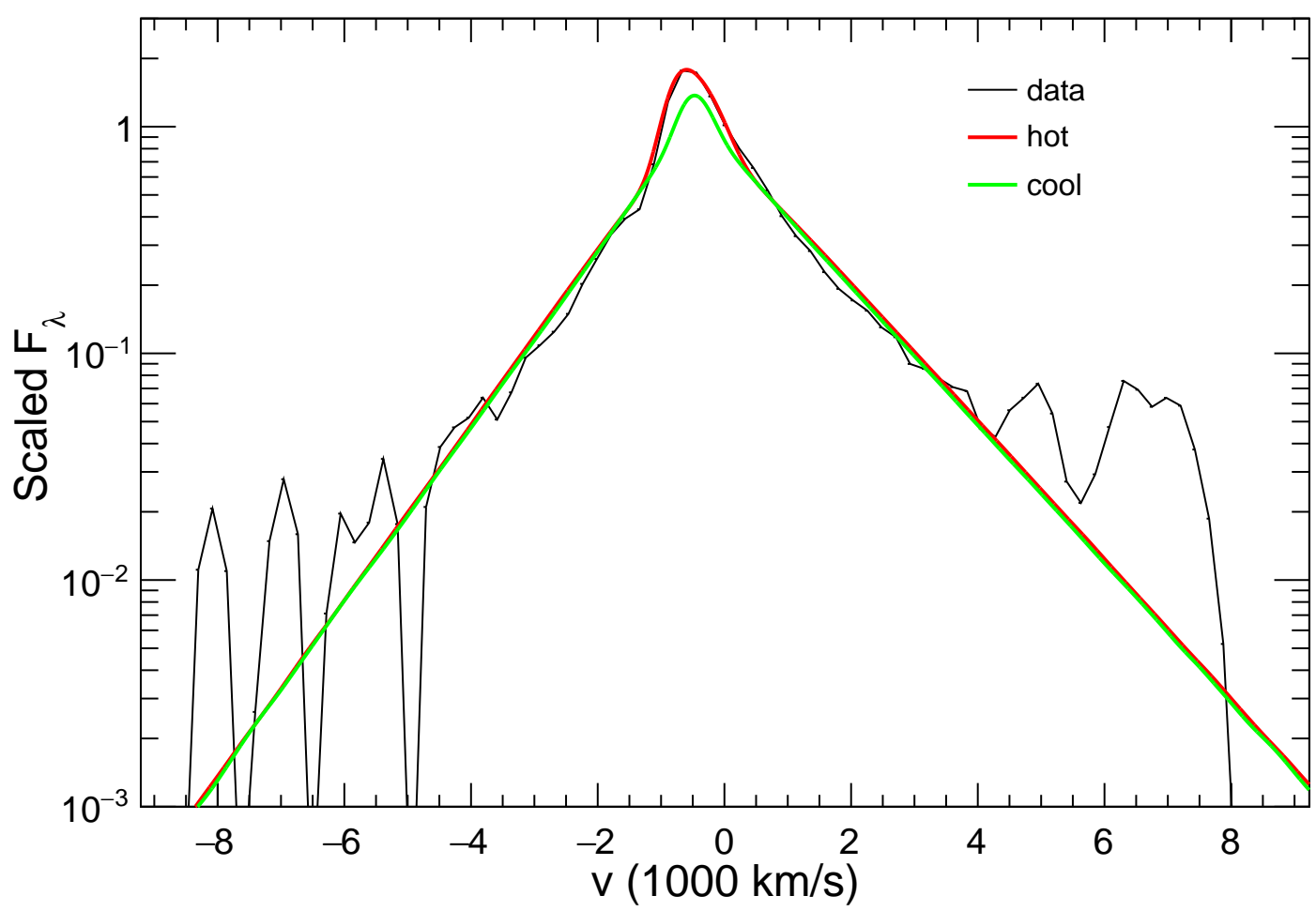

Fig. 3.16. - Comparison of the electron scattering model result with the SN2005db $\mathrm{H} \alpha$ emission line on 2005 August 14 (Kiewe et al. 2012). The parameters for the two models are given in Table 3.2.

Kiewe et al. (2012) observed, one of them, SN 2005cp, had emission that skewed the line profile to the blue, inconsistent with scattering in an outflowing wind. However, the emission is to the blue and the line profile is not consistent with an exponential. In this case, we conjecture that blue emission is due to the systematic Doppler shift of the emission. We did not model spectra of the fourth Type II supernova observed by Kiewe et al. (2012), SN 2005bx, because of the poor signal-to-noise.

\subsubsection{SN 2005gj, SN 2008J, and SN 2008cg}

The supernovae SNe 2005gj, 2008J, and 2008cg belong to the class of Type Ia-CSM supernovae, which show clear features of a Type Ia spectrum, as well as IIn charac- 


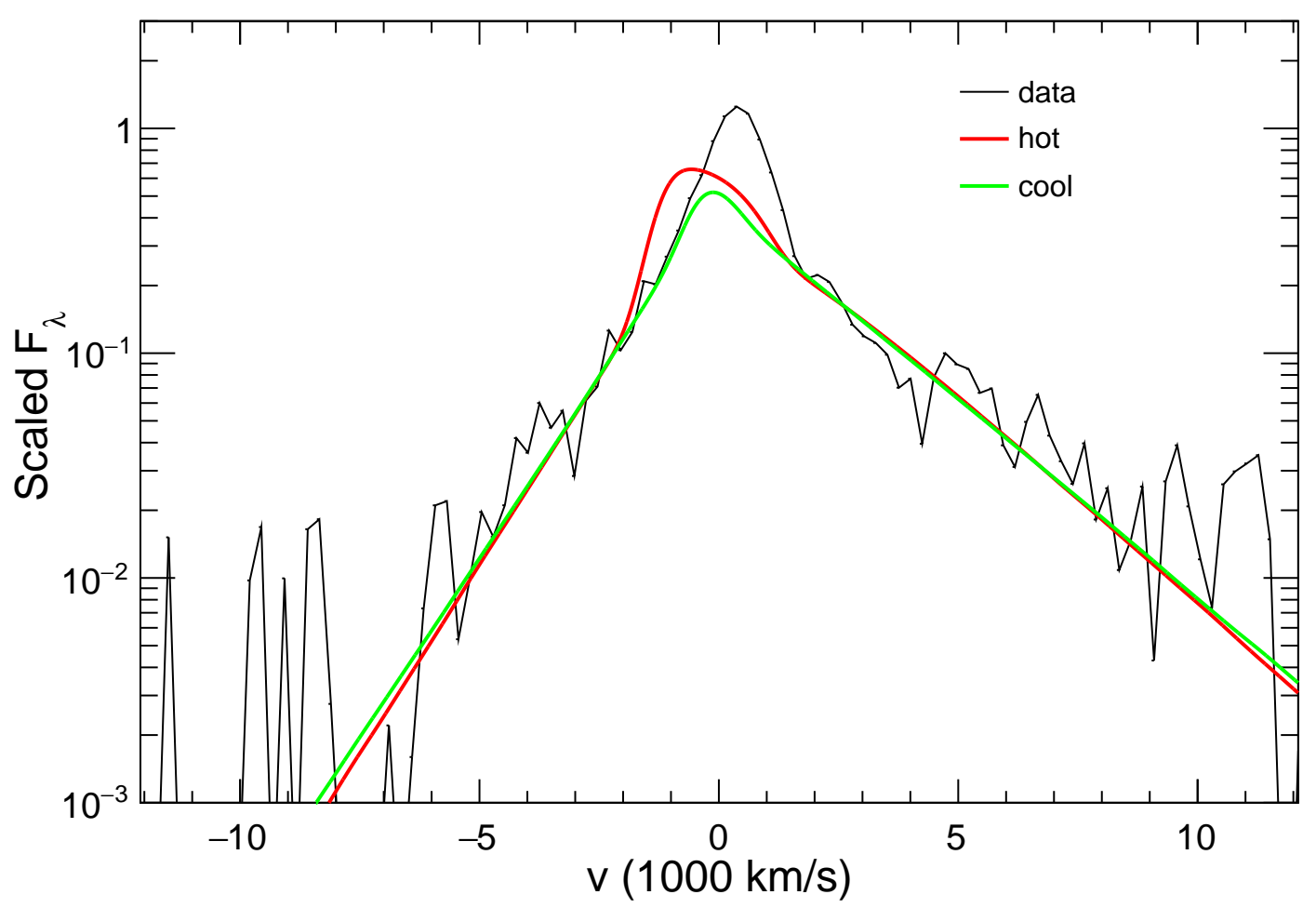

Fig. 3.17. - Comparison of the electron scattering model result with the SN 2012bq $\mathrm{H} \alpha$ emission line on 2012 April 12 (see text for details). The parameters for the model are given in Table 3.2

teristics (Hamuy et al. 2003; Silverman et al. 2013). The Type Ia features typically grow stronger with age of the supernova.

SN 2005gj was discovered on 2005 September 26 when it had an estimated age of 4 days since explosion (Aldering et al. 2006). Aldering et al. (2006) show fits of an electron scattering model to spectra on days 11, 64, and 71 after the explosion date; the fits are reasonable. Fig. 3.18 shows a spectrum taken with Keck II on 2005 December 2 with a resolution of $\sim 3 \AA$ (Silverman et al. 2013); the age is 67 days after discovery, or 71 days after explosion. The spectrum at that time shows a very broad feature roughly centered on $\mathrm{H} \alpha$ that cannot be explained with the electron scattering model. Fig. 7 of Aldering et al. (2006) makes clear that the feature is connected with 
a SN 1991T-type spectrum, a luminous subclass of Type Ia supernovae. Fig. 3.18 shows a comparison with our Monte Carlo electron scattering model. In addition to the blackbody spectrum, a Gaussian modeling the Type Ia feature near the $\mathrm{H} \alpha$ line has been subtracted off. Check The model parameters are in Table 3.2. The wind velocity from the $\mathrm{P}$ Cygni feature is estimated to be $\sim 300 \mathrm{~km} \mathrm{~s}^{-1}$ (Aldering et al. 2006; Prieto et al. 2007), and we used that value in our model. There is a small asymmetry in the observed broad line profile that is captured by the model with a wind velocity.

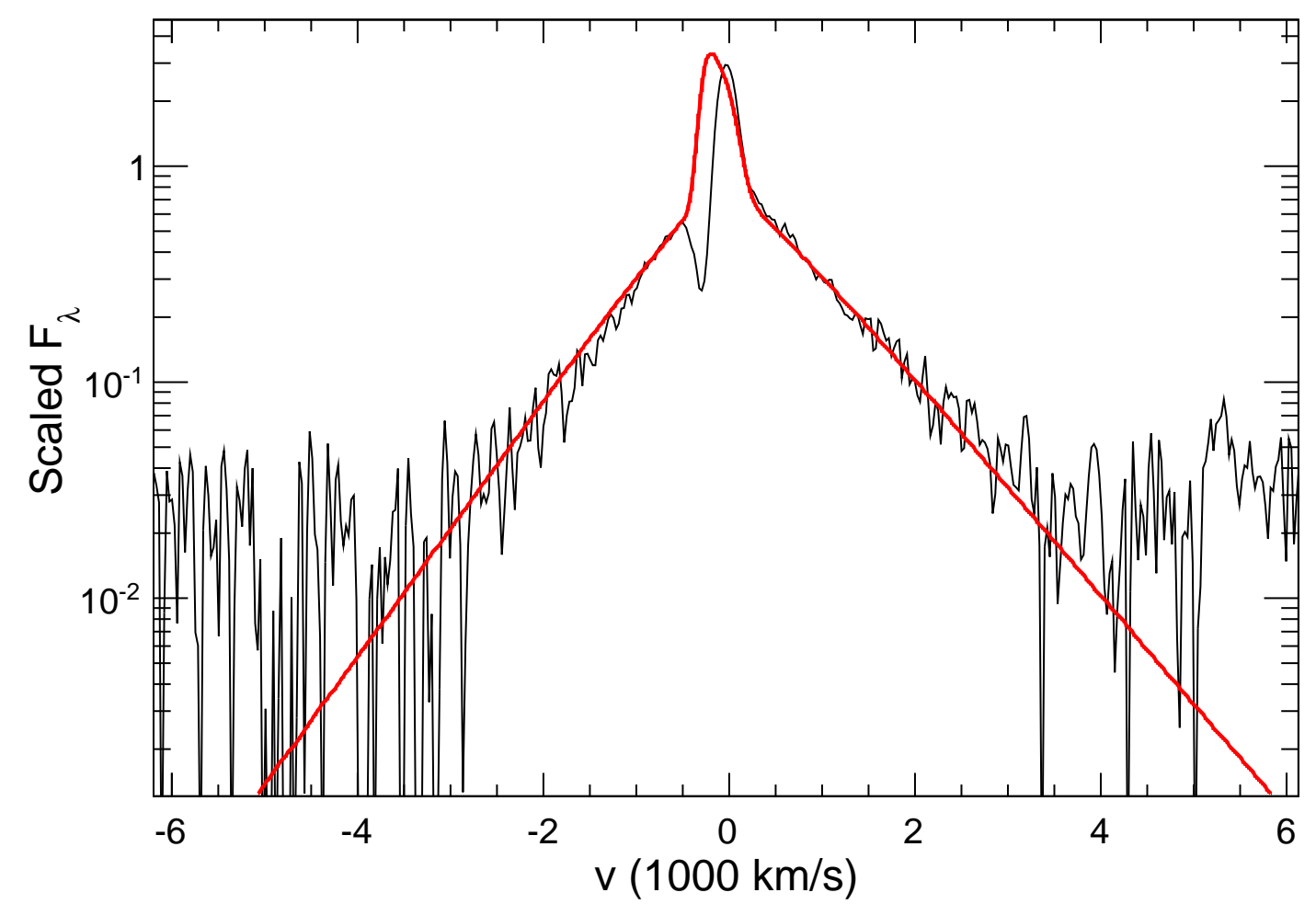

Fig. 3.18. - Comparison of the electron scattering model result with the SN 2005gj $\mathrm{H} \alpha$ emission line on 2005 December 2 (Silverman et al. 2013).

The spectrum of SN 2008J in Fig. 3.19 is from 2008 January 17, which is 2 days after the discovery of the supernova and 5.8 days before maximum $B$ light (Taddia et al. 2012). The spectral resolution is $\sim 6-9 \AA$. Taddia et al. (2012) find that 
the SN Ia features that appear are of the SN 1991T - type, as in SN 2005gj. The spectrum here is early, which is presumably why it gives a reasonable fit without an additional Gaussian feature; the features due to a Type Ia have not yet had time to grow. Any asymmetry in the line is small and our model has a small wind velocity.

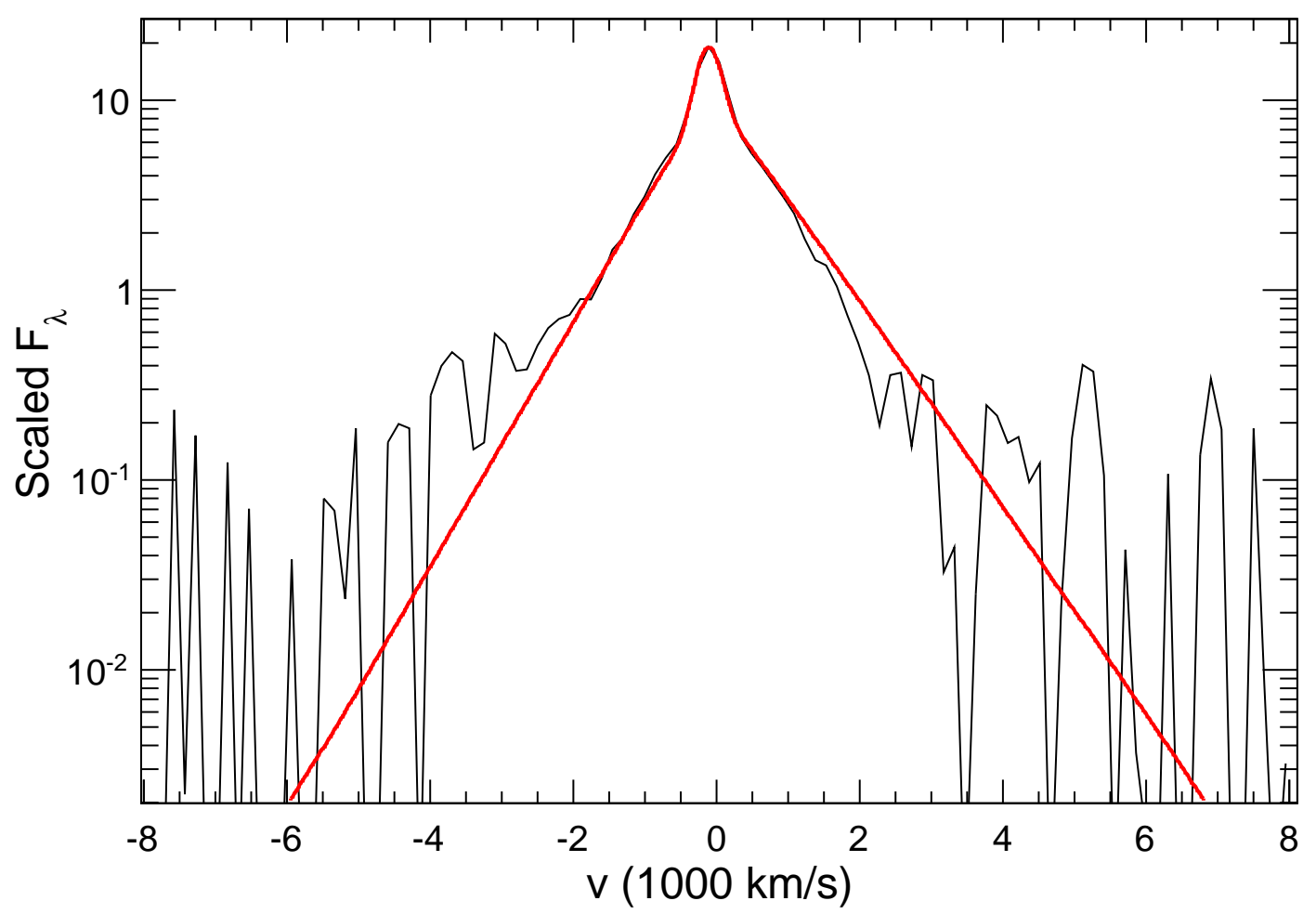

Fig. 3.19.- Comparison of the electron scattering model result with the SN 2008J H $\alpha$ emission line on 2008 January 17 (Taddia et al. 2012).

Fig. 3.20 shows a spectrum of SN 2008cg taken at the Lick 3m telescope on 2008 May 8, which Silverman et al. (2013) estimate to be 9 days after maximum light, or 3 days from discovery. The supernova is identified by Silverman et al. (2013) as Type Ia-CSM. This is not so clear from the early spectrum, but at later times the evidence seems quite clear. Silverman et al. (2013) note that initially the spectrum of SN 2008cg resembles a normal Type IIn event but after about 2 months, it closely resembles SN 2005gj. 


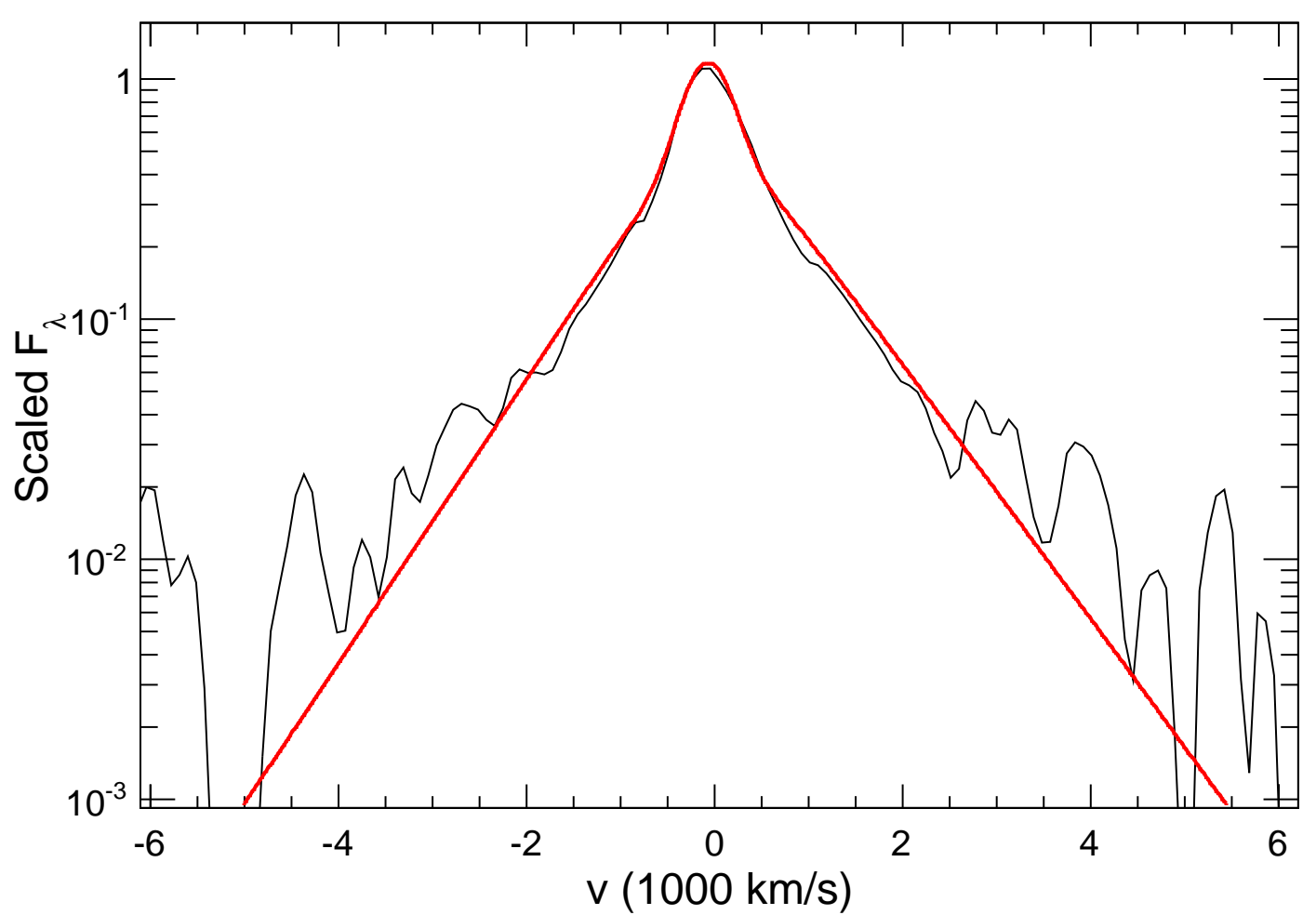

Fig. 3.20.- Comparison of the electron scattering model result with the SN $2008 \mathrm{cg}$ H $\alpha$ emission line on 2008 May 5 (Silverman et al. 2013).

We thus find that SNe Ia-CSM have $\mathrm{H} \alpha$ line wings that can be fit by an electron scattering profile. The line profiles are either symmetric or show a small asymmetry to the red, and are superposed on broad Type Ia supernova features that grow in strength with age. Silverman et al. (2013) find that the $\mathrm{H} \alpha$ lines in most SNe IaCSM show a deficit in the red wing starting at $\sim 75-100$ days after maximum light, later than the ages of the spectra considered here. This development is commonly attributed to the formation of dust which absorbs emission from gas moving away on the far side of the supernova. This interpretation is not compatible with line formation by electron scattering by thermal electrons, so the supernovae would have to begin a new phase of evolution. 


\subsubsection{SN 2009ip}

SN 2009ip is a massive star that underwent an unusual period of bursting activity covering years (Mauerhan et al. 2013; Margutti et al. 2014). There was an outburst in 2012 September, known as the 2012b event, in which it reached an absolute magnitude of -18 and showed emission line velocities of $\sim 10^{4} \mathrm{~km} \mathrm{~s}^{-1}$, which are characteristic of supernovae. Smith et al. (2014) find that persistent broad emission lines in the spectrum require an ejecta mass and kinetic energy that are characteristic of supernovae. However, Fraser et al. (2015) find no conclusive evidence for a core collapse supernova from the time of the outburst to 820 days later. Fig. 3.21 is from the Multiple Mirrowr Telescope (MMT), with spectral resolution R=5000 on 2012 Oct 14 (Margutti et al. 2014).

The emergence of the $\mathrm{H} \alpha$ line was presumably related to ionizing radiation related to shock interactions in the circumstellar medium. We were unable to fit the wings on the Holine with an electron scattering model. The excess in the line wing cannot be explained by an uncertainty of a blackbody background. The line wings do not have the approximately exponential profile expected for electron scattering. In addition, there is no inflection observed between the line core and line wing as expected in the electron scattering model, even though the narrow component is well resolved. However, the entire line profile is close to a modified Lorentzian profile although there is no physical rationale for this profile. A modified Lorentzian profile with a power law index 1.2 is shown in blue dotted line in Fig. 3.21.

\subsubsection{SN 2010jl}

SN 2010jl was an especially well observed Type IIn supernova, showing line profiles that are in agreement with electron scattering (Fransson et al. 2014; Borish et al. 


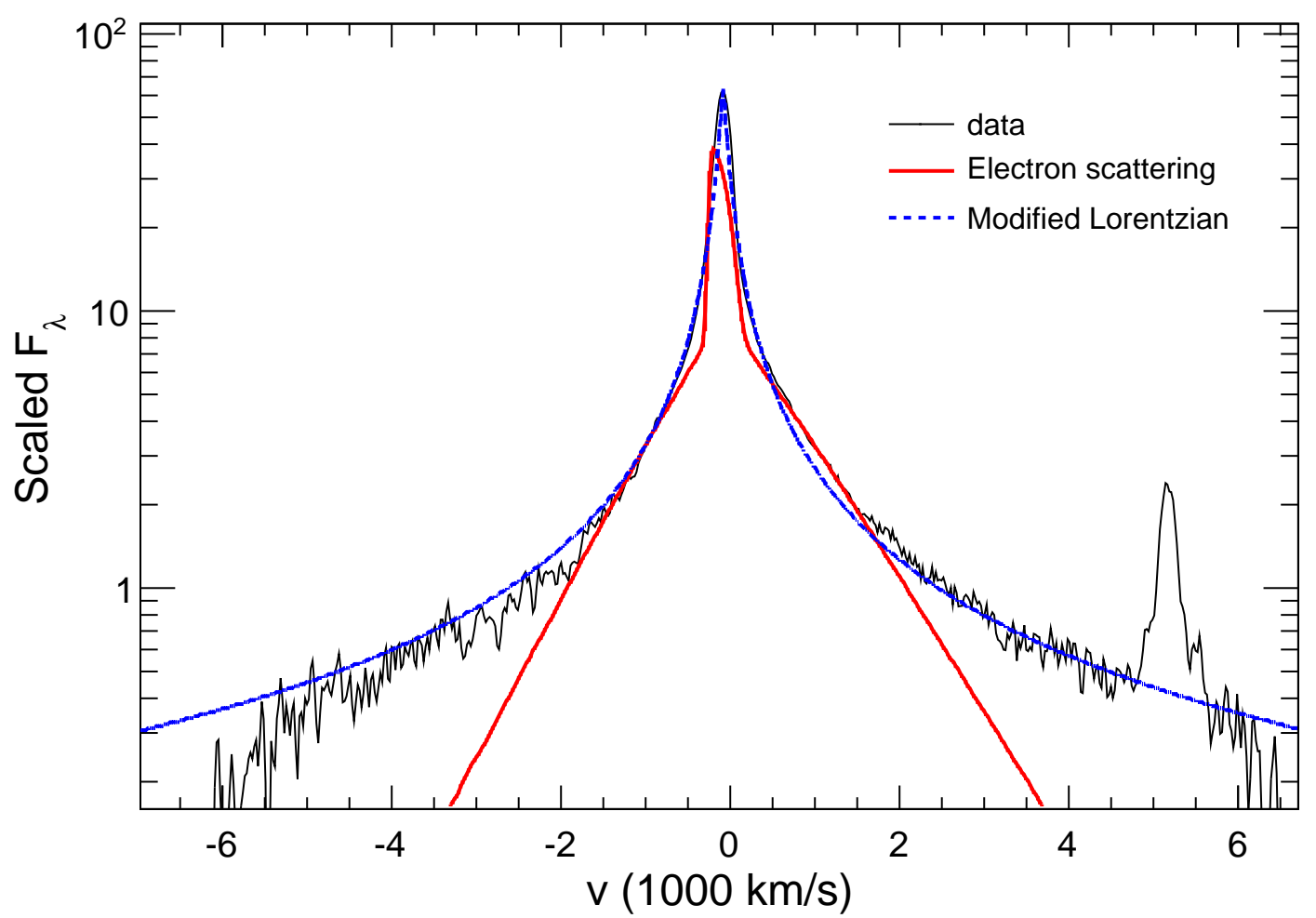

Fig. 3.21. - Comparison of the electron scattering model result and the modified Lorentzian with the SN 2009ip H $\alpha$ emission line on 2009 October 14 (Margutti et al. 2014).

2015). The $\mathrm{Pa} \beta$ line has a smoother background continuum than the $\mathrm{H} \alpha$ line, and the P-Cygni feature present in $\mathrm{H} \alpha$ does not show up in the narrow component of $\mathrm{Pa} \beta$. We thus chose the $\mathrm{Pa} \beta$ line to model in this case.

Fig. 3.22 shows the $\mathrm{Pa} \beta$ line. The narrow $\mathrm{H} \alpha$ line observed in SN 2010jl indicates $v_{w}=100 \mathrm{~km} \mathrm{~s}^{-1}$ (Fransson et al. 2014). This small wind velocity is consistent with the observed approximately symmetric scattering wings.

Fransson et al. (2014) and Borish et al. (2015) show that the broad component of Balmer lines shifts to the blue, but the narrow lines do not shift in the later time spectrum. It is plausible that the scattering region is distinct from the narrow line production. The evolution suggests that mass motions come to play a role in the line 


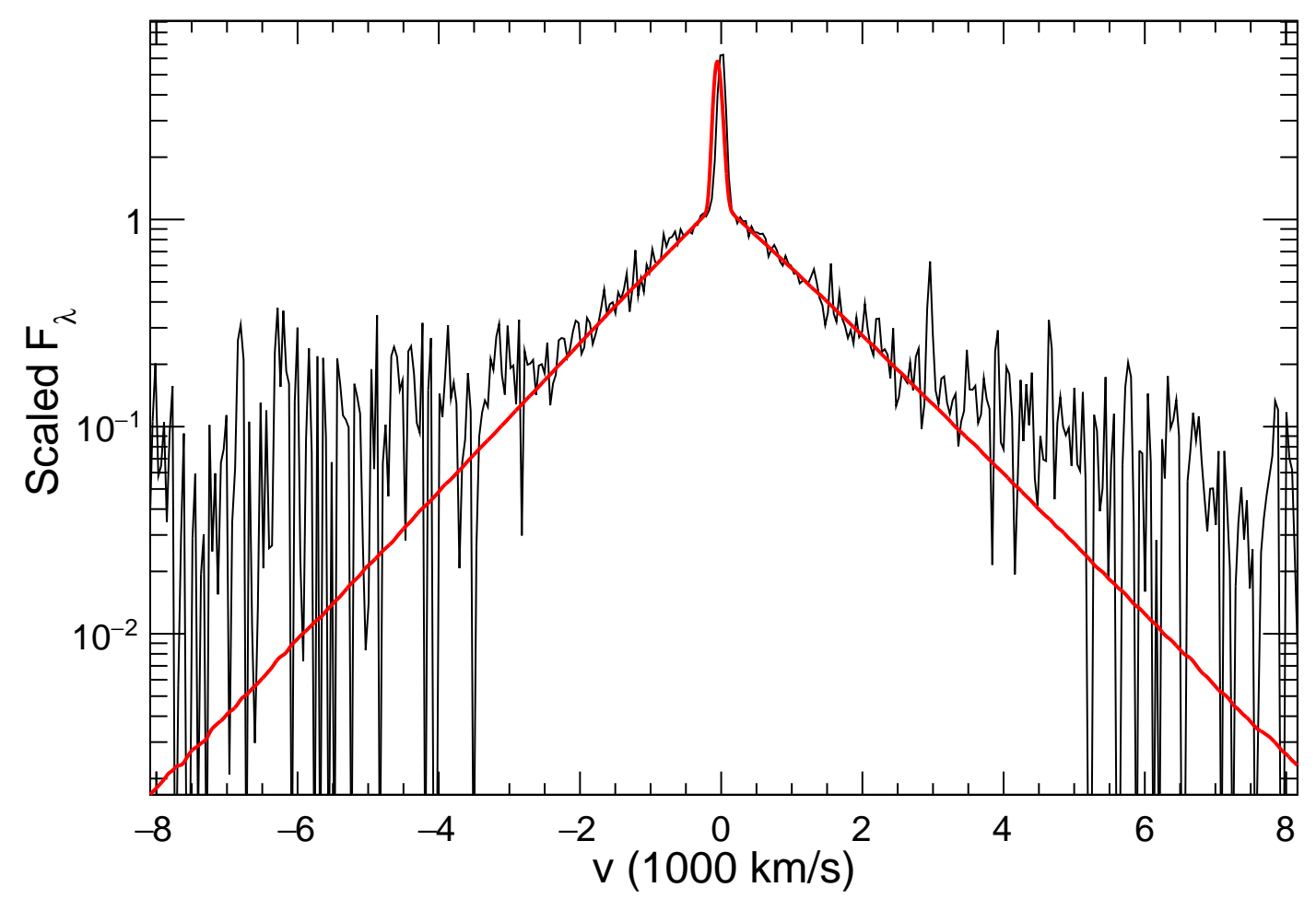

Fig. 3.22.- Comparison of the electron scattering model result with the SN 2010jl $\mathrm{Pa} \beta$ emission line on 2010 November 15 (Borish et al. 2015).

formation (e.g., Dessart et al. 2015).

\subsubsection{SN 2011ht}

We modeled an $\mathrm{H} \alpha$ spectrum obtained on 2011 Nov 11 from the Apache Point Observatory (APO) $3.5 \mathrm{~m}$ (Roming et al. 2012), which was the earliest spectrum available. Discovery was on 2011 Sept 29, so this is moderately late. Humphreys et al. (2012) suggested that this may not be a supernova, but rather a giant eruption event, because the kinetic energy of its ejecta seems much less than a regular supernova.

The Balmer emission lines show very broad wings with asymmetry to the red, which is a classic electron scattering signature. Later higher resolution spectra showed 
clear evidence for P Cygni line and a wind velocity of $600 \mathrm{~km} \mathrm{~s}^{-1}$ (Humphreys et al. 2012).

Fig. 3.23 shows a fit with $v_{w}=600 \mathrm{~km} \mathrm{~s}^{-1}$ that in good agreement with the observed asymmetry. The spectrum from WISeREP has been corrected for the galactic redshift of 0.0036. An extra redshift of 0.0005 was applied in the fitting. The SN 2011 ht spectrum has two strong and very broad He I emission lines at $5876 \AA$ and $7065 \AA$ (Humphreys et al. 2012), suggesting that the excess on the red wing from $3000 \mathrm{~km} \mathrm{~s}^{-1}$ to $7000 \mathrm{~km} \mathrm{~s}^{-1}$ is likely to be a broad He I 6678 feature.

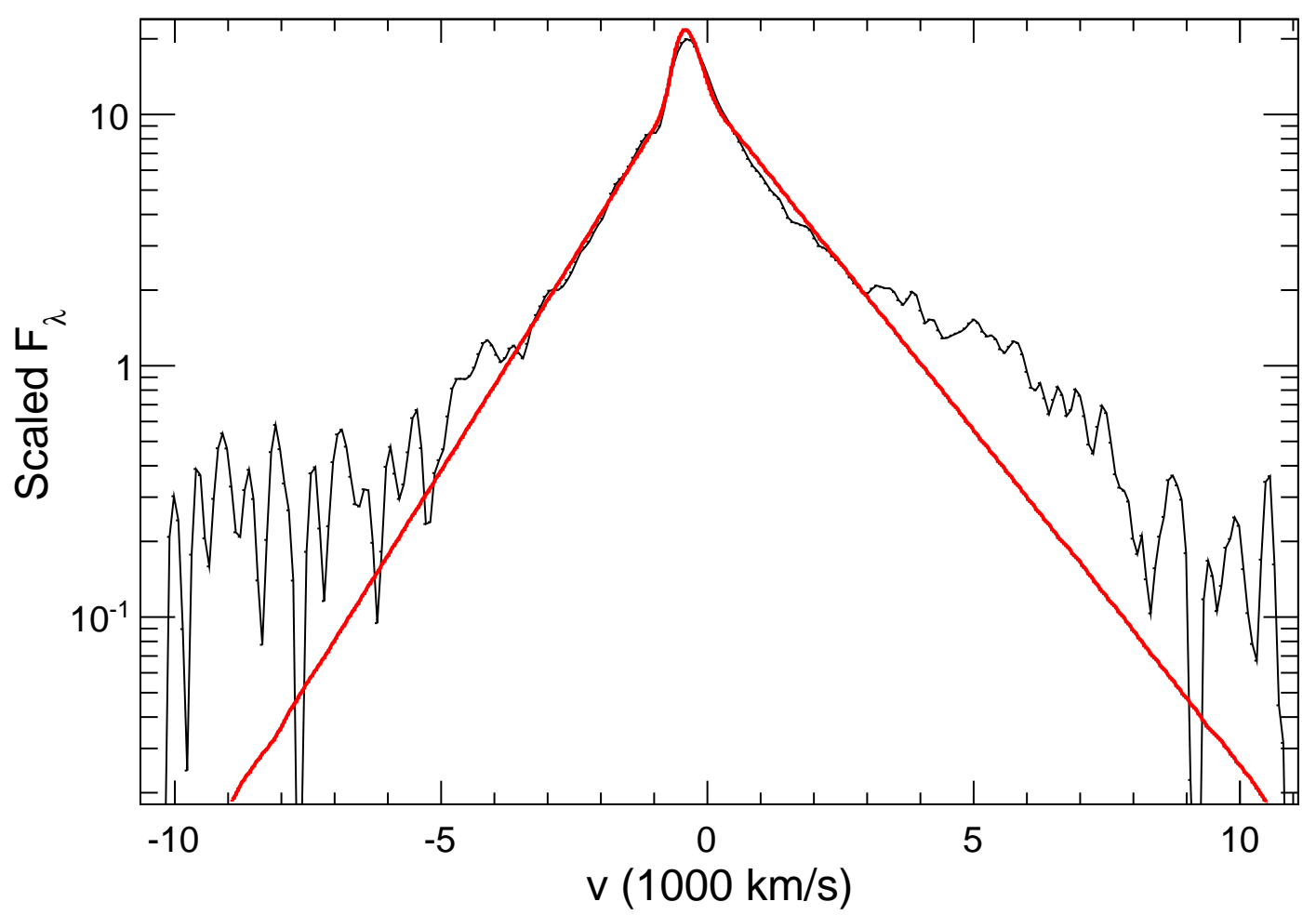

Fig. 3.23.- Comparison of the electron scattering model result with the SN 2011ht $\mathrm{H} \alpha$ emission line on 2011 November 11 (Roming et al. 2012). 


\subsection{Discussion and Conclusions}

The comparison of the electron scattering model with observations shows that scattering provides a plausible explanation for the line profile wings in many supernovae designated as Type IIn. It has been noted on occasion that a Lorentzian or modified Lorentzian profile gives a good approximation to the profiles observed in SNe IIn (Leonard et al. 2000; Shivvers et al. 2015), but there is no physical reason to expect such a profile. There has also been fitting by multiple Gaussians (e.g., Kiewe et al. 2012), but again there is not a clear physical explanation for such a profile.

An expectation of the electron scattering model is that there should be enhanced emission on the red side of the line if the scattering occurs in an extended surrounding medium with an outflow velocity. This feature is generally not observed. In the case of SN 1998S, there is evidence for dense mass loss occurring a short time before the explosion (Chugai 2001) and, thus, a limited extent scattering region which is consistent with the symmetry of the $\mathrm{H} \alpha$ line. The lines observed in SNe Ia-CSM are also symmetric over the first 2 months but, in this case, the supernova light curves do not indicate a late mass loss phase. The observations imply that the scattering remains in a fairly narrow region, although it is expanding outward with time. In the case of the SN 2005cl group of objects, there is an asymmetry with stronger emission to the red and these cases can be modeled with an outflowing scattering region. The observation of the asymmetry may be related to the high circumstellar outflow velocities found in these objects through their P Cygni profiles (Kiewe et al. 2012).

To a first approximation, the line profile resulting from electron scattering has an exponential shape. At low optical depths, the line profile has a concave shape, while at high optical depth it is convex. However, the differences only become clear 
far out in the wings, where it is not possible to obtain accurate observational data. The observed line profile shapes are not able to determine the value of $\tau$, so in the models there is a degeneracy in $\tau$ and $T$ in fitting the observed line width. If the line formation were optically thin in the line, the ratio of narrow line component to broad would break the degeneracy; however, the common observation of P Cygni features in the narrow line and the narrow line shape show that is not the case. In the models we present here, we made the assumption that the narrow to broad line ratio equals the optically thin ratio. Most of the model fits have $T$ is the $5000-20,000 \mathrm{~K}$ range, which is close to the temperatures expected in the photoionized gas and implies that the narrow to broad line ratio is not far from the optically thin case. However, models of the supernovae SN 2005cl, SN 2005db, and SN 2012bq yield a high temperature and relatively low optical depth. The implication is that the narrow to broad ratio is higher than it would be in the optically thin limit.

The determination of the outer wings of the lines depends sensitively on the assumed continuum. We have found that it is necessary to cover a broad wavelength range to obtain a reliable continuum fit. In the case of the SNe-CSM, there is structure in the continuum due to the underlying Type Ia spectrum that leads to uncertainty in the continuum fit. The strength of the feature near $\mathrm{H} \alpha$ grows with age. 
Table 3.1: Supernova observational parameters

\begin{tabular}{|c|c|c|c|c|c|c|c|}
\hline SN & $\begin{array}{c}\text { Date of } \\
\text { Observation }\end{array}$ & $\begin{array}{c}\text { Age } \\
\text { (days) }\end{array}$ & $\begin{array}{c}\text { Redshift } \\
z\end{array}$ & $\begin{array}{l}\text { Resolution } \\
(\AA)\end{array}$ & $\begin{array}{c}T_{b} \\
(\mathrm{~K})\end{array}$ & $E(B-V)$ & Ref. \\
\hline \multirow{2}{*}{$1998 \mathrm{~S}$} & 1998 Mar 4 & 1.9 & \multirow{2}{*}{0.00286} & 0.2 & 28,000 & \multirow{2}{*}{0.23} & $(1)$ \\
\hline & 1998 Mar 6 & 4 & & 8 & 28,000 & & $(2),(3)$ \\
\hline $2005 \mathrm{cl}$ & 2005 Jul 16 & 44 & 0.0259 & 5 & 19,000 & 0.4 & (4) \\
\hline $2005 \mathrm{db}$ & 2005 Aug 14 & 36 & 0.0151 & 5 & 6200 & 0.3 & (4) \\
\hline $2012 \mathrm{bq}$ & 2012 Apr 12 & 13 & 0.0415 & 18 & 12,000 & 0.2 & $(5)$ \\
\hline $2005 \mathrm{gj}$ & 2005 Dec 2 & 71 & 0.0616 & 3 & 10,000 & 0.4 & (6) \\
\hline 2008J & 2008 Jan 17 & 2 & 0.0159 & 7 & 10,000 & 0.8 & (7) \\
\hline $2008 \mathrm{cg}$ & 2008 May 5 & 3 & 0.0362 & 11.6 & 9000 & 0.2 & (6) \\
\hline 2009ip & 2012 Oct 14 & 21 & 0.00572 & 1.3 & 11,000 & 0.019 & $(8)$ \\
\hline $2010 j l$ & 2010 Nov 15 & 36 & 0.0107 & 4.3 & 5500 & 0.058 & (9) \\
\hline 2011ht & 2011 Nov 11 & 43 & 0.0036 & 7 & 13,000 & 0.062 & (10) \\
\hline
\end{tabular}

Note. - The ages are from the time of discovery. The listed redshifts of $2005 \mathrm{cl}, 2005 \mathrm{db}, 2005 \mathrm{gj}$, $2008 \mathrm{~J}, 2008 \mathrm{cg}, 2010 \mathrm{jl}$, and $2011 \mathrm{ht}$ are the measured values for the host galaxies. The redshift of 2012bq and 2009ip are measured from the peaks of the Balmer emission lines of the supernovae. The spectrum of $2011 \mathrm{ht}$ is redshift corrected.

References. - (1) Shivvers et al. (2015); (2) Leonard et al. (2000); (3) Fassia et al. (2001); (4) Kiewe et al. (2012); (4); (5) The spectrum is not published but is available in the WISeREP database; (6) Silverman et al. (2013); (7) Taddia et al. (2012); (8) Margutti et al. (2014); (9) Borish et al. (2015); (10) Humphreys et al. (2012) 
Table 3.2: Supernova model parameters used in fits

\begin{tabular}{lccccccc}
\hline \hline SN & $\begin{array}{c}\text { Model } \\
\text { redshift }\end{array}$ & $\begin{array}{c}\text { FWHM } \\
\left(\mathrm{km} \mathrm{s}^{-1}\right)\end{array}$ & $\begin{array}{c}\text { Radius } \\
\text { ratio } \eta\end{array}$ & $\tau$ & $\begin{array}{c}T_{e} \\
(\mathrm{~K})\end{array}$ & $\begin{array}{c}v_{w} \\
\left(\mathrm{~km} \mathrm{~s}^{-1}\right)\end{array}$ & $\begin{array}{c}v_{s h} \\
\left(\mathrm{~km} \mathrm{~s}^{-1}\right)\end{array}$ \\
\hline 1998S (day 2) & 0.00286 & 1600 & 3 & 1.6 & 22,000 & 40 & 200 \\
\hline 1998S (day 4) & 0.00286 & 2600 & 3 & 4 & 24,000 & 40 & 500 \\
\hline \multirow{2}{*}{$2005 \mathrm{cl}$} & 0.0275 & 3500 & 2 & 1.5 & 80,000 & 800 & 0 \\
\cline { 2 - 8 } $2005 \mathrm{db}$ & 0.0293 & 3200 & 1.2 & 5 & 18,000 & 1300 & 0 \\
\hline \multirow{2}{*}{$2012 \mathrm{bq}$} & 0.0163 & 2400 & 1.4 & 2 & 24,000 & 900 & 0 \\
\hline $2005 \mathrm{gj}$ & 0.0158 & 1900 & 1.4 & 6 & 6000 & 500 & 0 \\
\hline $2008 \mathrm{~J}$ & 0.0425 & 3500 & 10 & 2 & 60,000 & 1300 & 0 \\
\hline $2008 \mathrm{cg}$ & 0.041 & 3100 & 2 & 6 & 13,000 & 1000 & 0 \\
\hline $2009 \mathrm{ip}$ & 0.0621 & 1450 & 2 & 1.3 & 16,000 & 300 & 0 \\
\hline $2010 \mathrm{jl}$ & 0.0162 & 1200 & 2 & 2.5 & 7000 & 200 & 0 \\
\hline $2011 \mathrm{ht}$ & 0.036 & 1200 & 2 & 2 & 10,000 & 150 & 0 \\
\hline
\end{tabular}

Note. - The fit parameters for SN 2009ip are in parentheses because there is not a reasonable electron scattering model fit in this case. 


\section{Chapter 4}

\section{Summary and Future Work}

\section{$4.1 \quad$ Summary}

This thesis presents a spectral line formation study of two systems: the $\mathrm{H} \alpha$ and $\mathrm{NaD}$ lines in the hot Jupiter transmission spectrum (Chapters 1 and 2), and the broad wings of the emission lines of interacting supernovae (Chapter 3). By constructing theoretical models of these systems and comparing the simulated line profiles with observations, our understanding of these systems and related physical processes have been improved.

Chapter 1 introduced the transmission spectrum method used in constraining the properties of the exoplanetary atmosphere, summarized previous transmission spectrum observations of the upper atmosphere of the hot Jupiter HD 189733b, and explained the motivation for modeling the $\mathrm{H} \alpha$ and $\mathrm{NaD}$ transmission spectra.

As described in Chapter 2, a one-dimensional atmosphere model of the upper atmosphere of HD 189733b was constructed, with the goal of constraining its temperature, particle densities, and radiation field over the pressure range $10^{-4}-10 \mu$ bar, where the observed $\mathrm{H} \alpha$ transmission spectrum is produced. Because the upper atmo- 
sphere is not in thermal equilibrium, the atomic hydrogen level population is computed including both collisional and radiative transition rates (Section 2.2). The result shows that the $n_{2 p}$ is determined by the radiative rates between $1 s$ and $2 p$ throughout the simulation domain because of the large Ly $\alpha$ intensity. The $2 s$ and $2 p$ states reach collisional equilibrium by the large $p$ collisional $\ell$-mixing rate, which was overlooked in this context. This result is applied to the rest part of the model.

Ionization equilibrium and balance of heating and cooling processes are enforced at each level of the atmosphere model (Section 2.3). Metal species with solar abundance are included. Photoelectric heating is included using a synthetic spectrum for HD 189733, and secondary electron ionization and excitation are included. The temperature, and hence scale height, in the region optically thick to $\mathrm{H} \alpha$ is (roughly) set by a balance of photoelectric heating and line cooling by metal species, mainly $\mathrm{Mg} \mathrm{I}$ and $\mathrm{Na} \mathrm{I}$.

To obtain $\bar{J}_{L y \alpha}$ and calculate $n_{2 \ell}$, the Ly $\alpha$ resonant scattering is computed using a Monte-Carlo simulation (Section 2.4). $\bar{J}_{L y \alpha}$ is large and roughly constant down to the $P=0.1 \mu$ bar level of the atmosphere. Ly $\alpha$ photons created inside the atmosphere (converted from stellar LyC photons) and incident from the star are both important. For $P \gtrsim 0.1 \mu$ bar, $\bar{J}_{L y \alpha} \propto P^{-1}$.

The fiducial atmosphere model, discussed in Section 2.5, shows that the combination of the decreasing Ly $\alpha$ excitation rates and the increasing hydrogen density gives rise to a nearly flat $n_{2 \ell}$ over two decades in pressure. This layer is optically thick to $\mathrm{H} \alpha$, and the temperature is in the range $T \simeq 3000-8500 \mathrm{~K}$. Both $\mathrm{H} \alpha$ and $\mathrm{NaD}$ are optically thick up to the altitude with $P \sim 10^{-2} \mu$ bar, which corresponds to the atomic layer of the atmosphere. This leads to similar transit depths for the $\mathrm{H} \alpha$ and $\mathrm{Na} \mathrm{D}$ transmission lines, in agreement with the observations of Cauley et al. 
(2016) and Wyttenbach et al. (2015), suggesting that NaD line center is formed in the atomic layer above the molecular layer, while the line wing is formed in the molecular layer, as was assumed in previous studies (e.g., Huitson et al. 2012; Wyttenbach et al. 2015).

The model transmission spectra are in broad agreement with the data (Section 2.6). It is shown that the temperature achieved by fitting each wavelength interval in the observed transmission spectrum with an isothermal atmosphere model may not accurately retrieve the original temperature profile, if the temperature increases rapidly with the altitude. Additional models computed for a range of the stellar LyC flux suggest that the variability in $\mathrm{H} \alpha$ transit depth may be due to the variability in the stellar LyC. In contrast, the $\mathrm{Na}$ absorption profile is insensitive to the LyC level, which is a possible method to break the degeneracy between the transit depth variability due to blocking an active region on the star surface and the change in the atmosphere due to stellar activity. Since metal lines provide the dominant cooling of this part of the atmosphere, the atmospheric structure is sensitive to the density of species such as $\mathrm{Mg}$ and $\mathrm{Na}$, which may themselves be constrained by observations.

In Chapter 3, we model the electron scattering in the preshock CSM surrounding interacting supernovae, using a Monte Carlo scheme to calculate the effects of scattering in the Thomson limit. In the first part of the study (Section 3.2), we presented the main signature of the broadened line profile. A systematic scan over the parameter space shows the dependence of the line profiles on the optical depth, the geometric thickness, the density distribution, the continuum absorption, and the velocity profile of the CSM. The single scattering case gives the broad component in the limit of low optical depth, showing a velocity full width half maximum that is 
close to the thermal velocities of electrons. The line shape is approximately exponential at low velocities and steepens at higher velocities. For more general cases, the simulations were carried out in spherical symmetry. At higher optical depth, the line profile remains exponential at low velocities, but wings strengthen with increasing optical depth. In addition to the line width, the ratio of narrow to broad (scattered) line strength is a possible diagnostic of the gas. The radial outflow velocity of the circumstellar medium can lead to an asymmetry to the red if the circumstellar region is extended in radius. In the second part (Section 3.3), we apply the scattering model to a number of supernovae, including Type IIn and Type Ia-CSM events. For most supernovae which show broad wings on narrow emission lines in their spectrum, the emission line profiles show the signatures that are consistent with the electron scattering scenario. However, the emission line profile of the SN 2009ip shows different features compared with the electron scattering profile.

\subsection{Future Work}

\subsubsection{Temperature Profile Retrieval}

As mentioned in section 2.6.3, attempts to infer the atmosphere's temperature profile from the observed line profile have relied on the analytic formula (Equation 2.40) for isothermal atmospheres. However, it is expected that the line center is formed in the thermosphere where the temperature rapidly increases upward. Because of the result showing the deficiencies of the isothermal formula, an analytic atmosphere profile that can be easily used to interpret atmosphere properties from observed line profiles is needed.

Instead of an isothermal atmosphere, an atmosphere in which temperature de- 
creases with pressure or increases with altitude (as, e.g., a power law) would be a possible substitute. Such a simplified temperature profile would need to be physically motivated by numerically simulating line profiles need and comparing them with the one generated by the more realistic temperature profile (e.g., Figure 2.3). The resulting line profile can also be compared to the isothermal profile, enabling an analytical explanation for why the isothermal profile fails to reproduce the true temperature profile. An analytical approximation for the line profile, if it's possible to drive one, would greatly benefit future research.

\subsubsection{Solving the $\operatorname{Ly} \alpha$ Resonant Scattering Analytically.}

Although some limitations and approximations are to be expected, compared with Monte Carlo simulations, solving the problem analytically is much faster and provides a better understanding about the trend of Ly $\alpha$ intensity against optical depth, as well as the shape of Ly $\alpha$ spectrum. In addition, an approximate analytical expression can be easily coded into future exoplanet models in order to determine the impact of Ly $\alpha$ on cooling, heating, excitation states and radiation force etc. Thus, I will make effort to understand the Ly $\alpha$ resonant transfer analytically.

Harrington (1973) and subsequent works provided an analytical solution to resonant scattering problems in slab geometry. However, Harrington's solution is only approximate because the solution does not satisfy the boundary conditions of no incoming intensity, since the separation constant in the equation is allowed to be a function of the coordinates instead of a constant. Next, previous numerical investigations of resonant scattering have often used the spline fitting technique for the Hummer II-b redistribution function (Hummer 1962; Adams et al. 1971) in order to use fewer frequency points and decrease the computation time. However, this approx- 
imation of the redistribution function causes the scattering source and sink terms to only partially cancel each other, which limits the application of their result to the relatively large optical depth case. Using discretized sum over the Hummer II-b redistribution function (Hummer 1962) and solving the equation with the Feautrier method ensures the exact cancellation of the scattering source and sink terms. It allows the large optical depth case to be computed without artificially gaining or losing flux due to numerically-generated sources and it is more realistic than the isotropic scattering phase function used in Harrington (1973).

\subsubsection{Systematic Parameter Search and Simulating Other Observable Atomic Lines.}

With the launch of TESS and new ground based exoplanet transit surveys such as NGTS, thousands of new transiting exoplanets will be discovered. Unlike the host stars of Kepler planets, the target stars of these all-sky surveys are much brighter. Combined with powerful next generation ground based telescopes, there will be a large number of exoplanets that can be potential targets for transmission spectrum observations in the near future. A guide to which atmospheres are more likely to be observed through transmission spectroscopy is important.

In response to this requirement, and as a natural extension of my current project, I plan to conduct a systematic search for how stellar type, planet mass, planet radius, orbital radius and metalicity of planet atmospheres affect the transmission spectrum. Exoplanet atmospheres with higher temperature, less metalicity and weaker gravity tend to have larger scale heights, and thus puffier and easier to detect and characterize. Comparing the result of parameter search with the parameters of detected exoplanets, I can create a list of potential transmission spectroscopy target. Since the conditions 
of thermosphere sets the rate of gas escape, these results can also provide a restriction on the planet parameter space that can hold an atmosphere.

$\mathrm{H} \beta, \mathrm{H} \gamma$ absorption of HD 189733b (Cauley et al. 2016) and Balmer continuum absorption of HD 209458b (Ballester et al. 2007) have been detected. A model of these transitions can be made easily using the model. A prediction on absorption depth of Paschen series can also be made to guide future observations. Besides being the main cooling mechanism, the emission lines such as $\mathrm{Na}$ D doublet, $\mathrm{K}$ doublet, $\mathrm{MgI}$ 4571 and $\mathrm{MgI} 2853$ may also be detected directly. A simulation of the transmission spectrum of these lines can be conducted under the framework of my current model.

\subsubsection{Simulate the Ly $\alpha$ and FUV Flux Reaching the Molec- ular Layer}

Miguel et al. (2015) showed that the Ly $\alpha$ radiation changes atmospheric chemistry in the upper molecular layer of mini-Neptunes significantly, and $\mathrm{H}_{2} \mathrm{O}$ is most affected by Ly $\alpha$ radiation as the $\mathrm{H}_{2} \mathrm{O}$ photolysis rate strongly depends on the Ly $\alpha$ flux. They pointed out that studies of the absorption of Ly $\alpha$ flux and FUV flux in the exoplanet thermospheres are essential for realistic interpretation of planetary spectra.

I plan to simulate the Ly $\alpha$ and FUV flux at the base of thermosphere, which is the by-product of my code. In the previous studies on Ly $\alpha$ radiation in thermosphere (e.g. Koskinen et al. (2010)), people mostly focused on the scattering and absorption of stellar Ly $\alpha$ photon by the atmosphere. However, our results show that the external Ly $\alpha$ can barely penetrate the thermosphere because of the extremely large optical depth. In contrast, the photons generated deep inside the atmosphere, where is heated by the FUV and EUV stellar flux, are more likely to reach the molecular layer. In addition, the stellar FUV flux can also be strongly absorbed by the atoms 
of metal species in the thermosphere. Therefore, the simulation results may change the understanding of the molecular layer composition.

\subsubsection{Extending the Current Atmosphere Model to 3D.}

The current spherically symmetric atmosphere model, which assumes that the stellar radiation field is incident on the atmosphere vertically, is closest to the case of substellar region. However, atmosphere is not spherically symmetric because the star only illuminates the planet on one side. The atmosphere near the terminator is more important in terms of transmission spectrum.

To solve this problem, I plan to extend the current spherically symmetric atmosphere model to a true 3D atmosphere model using ZEUS code (Hayes et al. 2006). The microphysical processes, which determine the temperature and particle states of the atmosphere in my current result, will be included in the new 3D hydrodynamic model. Instead of simulating in the plane parallel geometry like I have, the Ly $\alpha$ resonant scattering simulation would be done in the 3D spherical grid generated by ZEUS. The results of a 3D Ly $\alpha$ intensity distribution makes it possible to compute the Ly $\alpha$ transmission spectrum, as well as the Ly $\alpha$ scattering spectrum, which also can potentially be observed.

\subsubsection{Explaining the $\mathrm{H} \alpha$ Absorption Leading the Transit.}

Cauley et al. $(2015,2016)$ observed a strong and unexpected $\mathrm{H} \alpha$ absorption feature lasting for a few hours before the HD 189733b transit. Bourrier et al. (2013) also observed Si III 1206.5 and N V 1240 pre-transit absorption in one of the two transits observed. If these observations are confirmed, they will not have a trivial explanation. A large population of $\mathrm{H}(n=2)$ cannot exist in an equilibrium state hydrogen outflow 
since the hydrogen would either be in the ground state if the temperature is low, or the hydrogen would be fully ionized if the temperature is high enough to excite a large amount of hydrogen. One possible explanation is that the $\mathrm{H}(n=2)$ are generated continuously in a strong active region, e.g the Balmer dominate shock (Heng \& McCray 2007) between a leading stream of the planet's atmosphere overflowing from L1 point and the stellar wind. These $\mathrm{H}(n=2)$ may produce the observed absorption feature before getting ionized or radiative decay. I will construct a model and simulate this process. 


\section{References}

Adams, T. F., Hummer, D. G., \& Rybicki, G. B. 1971, J. Quant. Spec. Radiat. Transf., 11,1365

Aldering, G., Antilogus, P., Bailey, S., et al. 2006, ApJ, 650, 510

Asplund, M., Grevesse, N., Sauval, A. J., \& Scott, P. 2009, ARA\&A, 47, 481

Auer, L. H., \& van Blerkom, D. 1972, ApJ, 178, 175

Ballester, G. E., Sing, D. K., \& Herbert, F. 2007, Nature, 445, 511

Barnes, J. R., Haswell, C. A., Staab, D., \& Anglada-Escudé, G. 2016, MNRAS, 462, 1012

Ben-Jaffel, L., \& Ballester, G. E. 2013, A\&A, 553, A52

Bildsten, L. 1998, in NATO Advanced Science Institutes (ASI) Series C, Vol. 515, NATO Advanced Science Institutes (ASI) Series C, ed. R. Buccheri, J. van Paradijs, \& A. Alpar, 419

Black, J. H., \& van Dishoeck, E. F. 1987, ApJ, 322, 412

Boisse, I., Moutou, C., Vidal-Madjar, A., et al. 2009, A\&A, 495, 959

Borish, H. J., Huang, C., Chevalier, R. A., et al. 2015, ApJ, 801, 7 
Borysow, A., Frommhold, L., \& Moraldi, M. 1989, ApJ, 336, 495

Bouchy, F., Udry, S., Mayor, M., et al. 2005, A\&A, 444, L15

Bourrier, V., Lecavelier des Etangs, A., Dupuy, H., et al. 2013, A\&A, 551, A63

Bowler, B. P. 2016, PASP, 128, 102001

Burrows, A. S. 2014a, Nature, 513, 345

—. 2014b, Proceedings of the National Academy of Science, 111, 12601

Cauley, P. W., Redfield, S., \& Jensen, A. G. 2017, AJ, 153, 217

Cauley, P. W., Redfield, S., Jensen, A. G., \& Barman, T. 2016, AJ, 152, 20

Cauley, P. W., Redfield, S., Jensen, A. G., et al. 2015, ApJ, 810, 13

Chandrasekhar, S. 1960, Radiative transfer

Charbonneau, D., Brown, T. M., Noyes, R. W., \& Gilliland, R. L. 2002, ApJ, 568, 377

Chatzopoulos, E., Wheeler, J. C., Vinko, J., et al. 2011, ApJ, 729, 143

Chevalier, R. A., \& Irwin, C. M. 2011, ApJ, 729, L6

Christie, D., Arras, P., \& Li, Z.-Y. 2013, ApJ, 772, 144

Chugai, N. N. 2001, MNRAS, 326, 1448

Cox, A. N. 2000, Allen's astrophysical quantities

Curdt, W., Tian, H., Teriaca, L., \& Schühle, U. 2010, A\&A, 511, L4 
Czesla, S., Klocová, T., Khalafinejad, S., Wolter, U., \& Schmitt, J. H. M. M. 2015, A\&A, 582, A51

Dalgarno, A., Yan, M., \& Liu, W. 1999, ApJS, 125, 237

Del Zanna, G., Dere, K. P., Young, P. R., Landi, E., \& Mason, H. E. 2015, A\&A, $582, \mathrm{~A} 56$

Deming, D., Wilkins, A., McCullough, P., et al. 2013, ApJ, 774, 95

Dere, K. P., Landi, E., Mason, H. E., Monsignori Fossi, B. C., \& Young, P. R. 1997, A\&AS, 125, doi:10.1051/aas:1997368

Dessart, L., Audit, E., \& Hillier, D. J. 2015, MNRAS, 449, 4304

Dessart, L., Hillier, D. J., Audit, E., Livne, E., \& Waldman, R. 2016, MNRAS, 458, 2094

Dessart, L., Hillier, D. J., Gezari, S., Basa, S., \& Matheson, T. 2009, MNRAS, 394, 21

Draine, B. T. 2011, Physics of the Interstellar and Intergalactic Medium

Drake, A. J., Djorgovski, S. G., Graham, M. J., et al. 2012, Central Bureau Electronic Telegrams, 3084

Ehrenreich, D., Bourrier, V., Bonfils, X., et al. 2012, A\&A, 547, A18

Fassia, A., Meikle, W. P. S., Chugai, N., et al. 2001, MNRAS, 325, 907

Fortney, J. J., Shabram, M., Showman, A. P., et al. 2010, ApJ, 709, 1396

Fransson, C., \& Chevalier, R. A. 1989, ApJ, 343, 323 
Fransson, C., Ergon, M., Challis, P. J., et al. 2014, ApJ, 797, 118

Fraser, M., Kotak, R., Pastorello, A., et al. 2015, MNRAS, 453, 3886

Gall, C., Hjorth, J., Watson, D., et al. 2014, Nature, 511, 326

García Muñoz, A. 2007, Planet. Space Sci., 55, 1426

Gladstone, G. R. 1988, J. Geophys. Res., 93, 14623

Hamuy, M., Phillips, M. M., Suntzeff, N. B., et al. 2003, Nature, 424, 651

Harrington, J. P. 1973, MNRAS, 162, 43

Hayes, J. C., Norman, M. L., Fiedler, R. A., et al. 2006, ApJS, 165, 188

Heng, K., \& McCray, R. 2007, ApJ, 654, 923

Hillier, D. J. 1991, A\&A, 247, 455

Huang, C., Arras, P., Christie, D., \& Li, Z.-Y. Submitted, Under review by ApJ

Huang, C., \& Chevalier, R. A. In preperation

Huitson, C. M., Sing, D. K., Vidal-Madjar, A., et al. 2012, MNRAS, 422, 2477

Hummer, D. G. 1962, MNRAS, 125, 21

Humphreys, R. M., Davidson, K., Jones, T. J., et al. 2012, ApJ, 760, 93

Igenbergs, K., Schweinzer, J., Bray, I., Bridi, D., \& Aumayr, F. 2008, Atomic Data and Nuclear Data Tables, 94, 981

Janev, R. K., Reiter, D., \& Samm, U. 2003, Collision processes in low-temperature hydrogen plasmas (Jülich: Forschungszentrum Jülich, Zentralbibliothek) 
Jensen, A. G., Redfield, S., Endl, M., et al. 2012, ApJ, 751, 86

—. 2011, ApJ, 743, 203

Kallman, T. R., \& McCray, R. 1982, ApJS, 50, 263

Katz, B., Sapir, N., \& Waxman, E. 2011, ArXiv e-prints, arXiv:1106.1898

Kiewe, M., Gal-Yam, A., Arcavi, I., et al. 2012, ApJ, 744, 10

Koskinen, T. T., Harris, M., Yelle, R. V., Lavvas, P., \& Lewis, N. 2011, in EPSC-DPS Joint Meeting 2011, 1169

Koskinen, T. T., Harris, M. J., Yelle, R. V., \& Lavvas, P. 2013a, Icarus, 226, 1678

Koskinen, T. T., Yelle, R. V., Harris, M. J., \& Lavvas, P. 2013b, Icarus, 226, 1695

Koskinen, T. T., Yelle, R. V., Lavvas, P., \& Lewis, N. K. 2010, ApJ, 723, 116

Kramida, A., Yu. Ralchenko, Reader, J., \& and NIST ASD Team. 2015, NIST Atomic Spectra Database (ver. 5.3), [Online]. Available: http://physics.nist.gov/asd [2016, January 31]. National Institute of Standards and Technology, Gaithersburg, MD.

Kreidberg, L., Bean, J. L., Désert, J.-M., et al. 2014, Nature, 505, 69

Landini, M., \& Fossi, B. C. M. 1991, A\&AS, 91, 183

Laor, A. 2006, ApJ, 643, 112

Lavvas, P., Koskinen, T., \& Yelle, R. V. 2014, ApJ, 796, 15

Lecavelier Des Etangs, A., Pont, F., Vidal-Madjar, A., \& Sing, D. 2008, A\&A, 481, L83 
Lecavelier Des Etangs, A., Ehrenreich, D., Vidal-Madjar, A., et al. 2010, A\&A, 514, A72

Lecavelier des Etangs, A., Bourrier, V., Wheatley, P. J., et al. 2012, A\&A, 543, L4

Lennon, M. A., Bell, K. L., Gilbody, H. B., et al. 1988, Journal of Physical and Chemical Reference Data, 17, 1285

Lenzuni, P., Chernoff, D. F., \& Salpeter, E. E. 1991, ApJS, 76, 759

Leonard, D. C., Filippenko, A. V., Barth, A. J., \& Matheson, T. 2000, ApJ, 536, 239

Lindholm, D. M., Ware DeWolfe, A., Wilson, A., et al. 2011, AGU Fall Meeting Abstracts

Linsky, J. L., France, K., \& Ayres, T. 2013, ApJ, 766, 69

Lucy, L. B. 1999, A\&A, 344, 282

Margutti, R., Milisavljevic, D., Soderberg, A. M., et al. 2014, ApJ, 780, 21

Mauerhan, J. C., Smith, N., Filippenko, A. V., et al. 2013, MNRAS, 430, 1801

Menager, H., Barthélemy, M., Koskinen, T., et al. 2013, Icarus, 226, 1709

Miguel, Y., Kaltenegger, L., Linsky, J. L., \& Rugheimer, S. 2015, MNRAS, 446, 345

Murray-Clay, R. A., Chiang, E. I., \& Murray, N. 2009, ApJ, 693, 23

Osorio, Y., Barklem, P. S., Lind, K., et al. 2015, A\&A, 579, A53

Osterbrock, D. E., \& Ferland, G. J. 2006, Astrophysics of gaseous nebulae and active galactic nuclei

Pequignot, D., \& Aldrovandi, S. M. V. 1976, A\&A, 50, 141 
Pequignot, D., Petitjean, P., \& Boisson, C. 1991, A\&A, 251, 680

Pillitteri, I., Maggio, A., Micela, G., et al. 2015, ApJ, 805, 52

Pillitteri, I., Wolk, S. J., Lopez-Santiago, J., et al. 2014, ApJ, 785, 145

Pont, F., Knutson, H., Gilliland, R. L., Moutou, C., \& Charbonneau, D. 2008, MNRAS, 385, 109

Pont, F., Sing, D. K., Gibson, N. P., et al. 2013, MNRAS, 432, 2917

Poppenhaeger, K., Schmitt, J. H. M. M., \& Wolk, S. J. 2013, ApJ, 773, 62

Pozdnyakov, L. A., Sobol, I. M., \& Syunyaev, R. A. 1983, Astrophysics and Space Physics Reviews, 2, 189

Press, W. H., Teukolsky, S. A., Vetterling, W. T., \& Flannery, B. P. 2007, Numerical Recipes 3rd Edition: The Art of Scientific Computing, 3rd edn. (New York, NY, USA: Cambridge University Press)

Prieto, J. L., Garnavich, P. M., Phillips, M. M., et al. 2007, ArXiv e-prints, arXiv:0706.4088

Redfield, S., Endl, M., Cochran, W. D., \& Koesterke, L. 2008, ApJ, 673, L87

Roming, P. W. A., Pritchard, T. A., Prieto, J. L., et al. 2012, ApJ, 751, 92

Rybicki, G. B., \& Lightman, A. P. 1979, Radiative processes in astrophysics

Salz, M., Czesla, S., Schneider, P. C., \& Schmitt, J. H. M. M. 2016, A\&A, 586, A75

Sanz-Forcada, J., Micela, G., Ribas, I., et al. 2011, A\&A, 532, A6

Schmitt, J. H. M. M. 2017, Astronomische Nachrichten, 338, 178 
Seager, S. 2010, Exoplanet Atmospheres: Physical Processes (Princeton University Press)

Seaton, M. J. 1955, Proceedings of the Physical Society A, 68, 457

Sharp, C. M., \& Burrows, A. 2007, ApJS, 168, 140

Shivvers, I., Groh, J. H., Mauerhan, J. C., et al. 2015, ApJ, 806, 213

Shull, J. M. 1978, ApJ, 224, 841

Shull, J. M., \& van Steenberg, M. 1982, ApJS, 48, 95

Silverman, J. M., Nugent, P. E., Gal-Yam, A., et al. 2013, ApJS, 207, 3

Sing, D. K., Fortney, J. J., Nikolov, N., et al. 2016, Nature, 529, 59

Smith, N., Chornock, R., Silverman, J. M., Filippenko, A. V., \& Foley, R. J. 2010, ApJ, 709, 856

Smith, N., Mauerhan, J. C., \& Prieto, J. L. 2014, MNRAS, 438, 1191

Smith, N., Silverman, J. M., Filippenko, A. V., et al. 2012, AJ, 143, 17

Sunyaev, R. A. 1980, Soviet Astronomy Letters, 6, 213

Taddia, F., Stritzinger, M. D., Phillips, M. M., et al. 2012, A\&A, 545, L7

Team, T. O. P. 1995, The Opacity Project Vol. 1, Institute of Physics Publications, Bristol, UK

Tian, H., Curdt, W., Marsch, E., \& Schühle, U. 2009, A\&A, 504, 239

van Regemorter, H. 1962, ApJ, 136, 906 
Verner, D. A., \& Ferland, G. J. 1996, ApJS, 103, 467

Verner, D. A., Ferland, G. J., Korista, K. T., \& Yakovlev, D. G. 1995, in Bulletin of the American Astronomical Society, Vol. 27, American Astronomical Society Meeting Abstracts \#186, 859

Verner, D. A., Ferland, G. J., Korista, K. T., \& Yakovlev, D. G. 1996, ApJ, 465, 487

Vidal-Madjar, A., Lecavelier des Etangs, A., Désert, J.-M., et al. 2003, Nature, 422, 143

Vidal-Madjar, A., Huitson, C. M., Bourrier, V., et al. 2013, A\&A, 560, A54

Visscher, C., Lodders, K., \& Fegley, Jr., B. 2010, ApJ, 716, 1060

Vrinceanu, D., Onofrio, R., \& Sadeghpour, H. R. 2012, ApJ, 747, 56

Weymann, R. J. 1970, ApJ, 160, 31

Whitney, B. A. 2011, Bulletin of the Astronomical Society of India, 39, 101

Wiese, W. L., \& Fuhr, J. R. 2009, Journal of Physical and Chemical Reference Data, 38,565

Winn, J. N. 2010, Exoplanet Transits and Occultations (University of Arizona Press), $55-77$

Wyttenbach, A., Ehrenreich, D., Lovis, C., Udry, S., \& Pepe, F. 2015, A\&A, 577, A62

Yaron, O., \& Gal-Yam, A. 2012, PASP, 124, 668

Yelle, R. V. 2004, Icarus, 170, 167

Zheng, Z., \& Miralda-Escudé, J. 2002, ApJ, 578, 33 